LUÍS FERNANDO NOGUEIRA DE SÁ

Topology Optimization Method applied to Laminar Flow Machine Rotor Design 
LUÍS FERNANDO NOGUEIRA DE SÁ

\section{Topology Optimization Method applied to Laminar Flow Machine Rotor Design}

Submitted in partial fulfilment of the requirements for the degree of Master of Science in Mechanical Engineering 
LUÍS FERNANDO NOGUEIRA DE SÁ

\section{Topology Optimization Method applied to Laminar Flow Machine Rotor Design}

Submitted in partial fulfilment of the requirements for the degree of Master of Science in Mechanical Engineering

Field of Study:

Control and Mechanical Automation Engineering (3152)

Supervised by:

Prof. Dr. Emílio Carlos Nelli Silva 
Este exemplar foi revisado e corrigido em relação à versão original, sob responsabilidade única do autor e com a anuência de seu orientador.

São Paulo, de de

Assinatura do autor:

Assinatura do orientador:

Catalogação-na-publicação

Sá, Luís Fernando Nogueira de

Topology Optimization Method applied to Laminar Flow Machine Rotor Design / L. F. N. Sá -- versão corr. -- São Paulo, 2016. $113 \mathrm{p}$.

Dissertação (Mestrado) - Escola Politécnica da Universidade de São Paulo. Departamento de Engenharia Mecatrônica e de Sistemas Mecânicos.

1.Métodos Topológicos (Otimização) 2.Bombas Centrífugas 3.Método dos Elementos Finitos 4.Dinâmica dos Fluidos (Simulação) I.Universidade de São Paulo. Escola Politécnica. Departamento de Engenharia Mecatrônica e de Sistemas Mecânicos II.t. 
In memory of my grandfather Hélio, who will always be present in my mind at every stage of my life. 


\section{ACKNOWLEDGEMENTS}

To my supervisor, Professor Dr. Emílio Carlos Nelli Silva, for the attention, discussions and suggestions given during the execution of this dissertation. His guidance and support were precious for the development of this work.

To my parents, Claudio N. de Sá and Roseli B. N. de Sá, and to my brother, André N. de Sá, not only for the education and formation proportioned, but also for the moments of happiness. I thank you all for the unconditional support to all my decisions. Also to Shiva, for the loyalty and caring. And in special to my grandfather, Hélio N. de Sá, for the life lessons and guidance on being a better person.

To my girlfriend Jacqueline Kian for the love, understanding and patience during the execution of this work.

To my closest friends Igor Meira, Jone Chen, Caio Gutierrez and Natália Salgado for the support and friendship.

To my laboratory colleagues Ricardo Amigo, Paulo Yamabe, Ruben Sallas, Sandro Vatanabe, Diego Prado, Mariana Moretti, César Kiyono and Francisco Oliveira for the academic discussions and companionship.

To Professor Oswaldo Horikawa for the aid in building the experimental site and the electric motor. Also, to Bruno Utiyama for the help in 3D printing the prototypes.

To Professor Juan Romero for the useful comments and for sharing information about his work.

To FAPESP (São Paulo Research Foundation) for the financial support to this research under grant 2015/15189-7. 
"Humankind cannot gain anything without first giving something in return. To obtain, something of equal value must be lost."

- Alphonse Elric 


\section{RESUMO}

Máquinas de fluxo são muito importantes para a indústria, sendo utilizadas em diversos processos. Assim, melhorias de desempenho são fatores relevantes e podem ser alcançadas com a utilização de métodos de otimização, como a otimização topológica. Este trabalho visa desenvolver uma metodologia para projetar rotores de máquinas de fluxo radiais que operam em escoamento laminar implementando-se a formulação de otimização topológica baseada no modelo de densidades. O projeto de rotores envolve, primeiramente, a modelagem do escoamento utilizando-se as equações de Navier-Stokes em um referencial rotativo e a utilização do Método de Elementos Finitos para a resolução das equações diferenciais. A distribuição de material no domínio é feita empregando-se um modelo de escoamento em meio poroso baseado nas equações de Darcy, utilizando-se a permeabilidade inversa que interpola o elemento entre sólido e fluido. Na fase de otimização é definida uma função multi-objetivo, que visa minimizar dissipação de energia viscosa, a vorticidade e a potência. O problema de otimização é implementado utilizando-se o ambiente FEniCS para a resolução do sistema de elementos finitos e as bibliotecas dolfin-adjoint e pyIpopt para o algorithmo de otimização. As topologias otimizadas são verificadas com o software ANSYS. As topologias resultantes são pós-processadas para a criação de um modelo CAD dos rotores. Os rotores são construídos utilizando-se a impressão 3D, o protótipo completo é montado acoplando-se um motor elétrico sem escovas e a caracterização experimental é feita medindo-se a vazão e o ganho de pressão dados pelas bombas. Por fim, os resultados experimentais e computacionais são comparados e uma melhoria de desempenho é observada.

Palavras-chave: Otimização Topológica. Máquinas de Fluxo Radial. Projeto de Rotores. Impressora 3D. Caracterização Experimental. 


\section{ABSTRACT}

Flow machines are very important to industry, being widely used on various processes. Performance improvements are relevant factors and can be achieved by using optimization methods, such as topology optimization. Thus, this work aims to develop a method to design radial flow machine rotors operating on laminar regime, by implementing a topology optimization formulation based on density model. The design of a rotor involves firstly modelling the fluid flow by using the Navier-Stokes equations on a rotating reference frame and using the Finite Element Method for solving the differential equations. To determine the material distribution on the domain, a porous flow model based on the Darcy equation is employed by using an inverse permeability that interpolates between fluid and solid. In the optimization phase, it is defined a multi-objective function that aims to minimize the viscous energy dissipation, vorticity and power. The optimization problem is implemented using the FEniCS environment and the libraries dolfin-adjoint and pyIpopt. The optimized topologies are verified with the ANSYS software. The resulting topologies are post-processed and a CAD model is created. The rotors are manufactured by using a $3 \mathrm{D}$ printer, the complete prototype is built by coupling an electric brushless motor and an experimental characterization is performed by measuring fluid flow and pressure head given by the pumps. Experimental and computational results are compared and the improvement is verified.

Keywords: Topology Optimization. Radial Flow Machine. Rotor Design. 3D Printer. Experimental Characterization. 


\section{LIST OF FIGURES}

Figure 1.1 - Radial Flow Machine Model . . . . . . . . . . . . . . . . . . . . . . 19

Figure 1.2 - Blade splitters . . . . . . . . . . . . . . . . . . . 20

Figure 1.3 - Three optimization approaches applied to design of a flow channel by considering minimization of pressure loss $\ldots \ldots \ldots \ldots . \ldots 21$

Figure 1.4 - Topology optimization method procedure for fluid domains . . . . . . 23

Figure 2.1 - Rotor Model approximation by using a Rotational Straight Channel

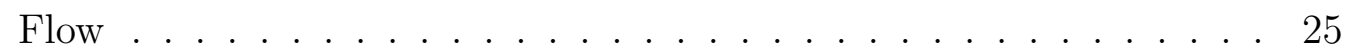

Figure 2.2 - Triangular Element Interpolation: (a)Pressure, (b)Velocity and (c)Design

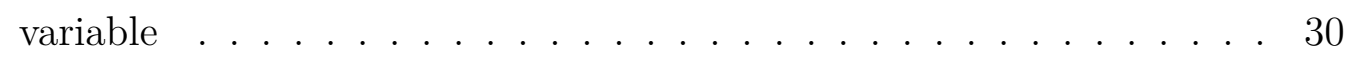

Figure 2.3 - Triangular element local coordinate frame $\ldots \ldots \ldots . . \ldots 30$

Figure 3.1 - Topology optimization applied to flow channel problem . . . . . . . . 31

Figure 3.2 - Topology optimization method applied to design of a straight blade rotor by considering energy dissipation as objective function . . . . . 32

Figure 3.3 - Complete cycle for development of a flow machine rotor by using

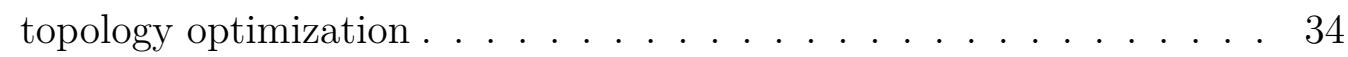

Figure $3.4-\kappa$ as function of parameters $\alpha$ and q $\ldots \ldots \ldots \ldots$

Figure 5.1 - Methods for obtaining the adjoint code . . . . . . . . . . . 52

Figure 5.2 - Topology optimization implementation flow chart . . . . . . . . 54

Figure $6.1-$ Design domain and boundary conditions $\ldots \ldots \ldots \ldots$

Figure $6.2-$ Straight blade initial guess $\ldots \ldots \ldots \ldots \ldots$. . . . . . . 57

Figure 6.3 - Topology optimization results by considering a straight blade as initial

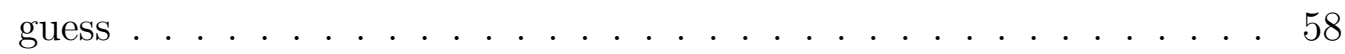

Figure 6.4 - Velocity fields for topology optimization results by considering a straight blade as initial guess . . . . . . . . . . . . . . . . . 59

Figure 6.5 - Entire rotors corresponding to each topology optimization result by considering a straight blade as initial guess . . . . . . . . . . . . 59

Figure 6.6 - Convergence curve for optimization with straight blade initial guess

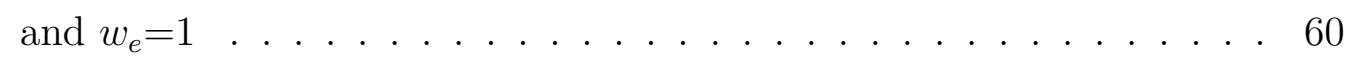

Figure 6.7 - Involute blade optimization $\ldots \ldots \ldots \ldots \ldots$. . . . . . . 61

Figure 6.8 - Optimization results by considering a involute blade as initial guess . 62

Figure 6.9 - Velocity fields for topology optimization results by considering a involute blade as initial guess $\ldots \ldots \ldots \ldots . \ldots \ldots 2$

Figure 6.10 - Entire rotors corresponding to each topology optimization result by considering a involute blade as initial guess . . . . . . . . . . . . 63

Figure 6.11 - Design domain and boundary conditions for turbine . . . . . . . . 64

Figure 6.12 - Involute blade optimization Initial domain $\ldots \ldots \ldots$. . . . . . 64 
Figure 6.13 - Velocity fields for turbine initial guess with rotations: (a) $20[\mathrm{rpm}]$ and

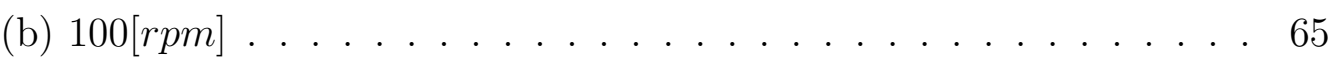

Figure 6.14 - Pressure fields for turbine initial guess with rotations: (a) $20[\mathrm{rpm}]$ and

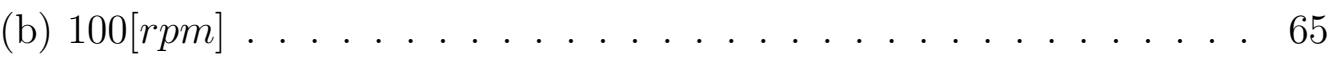

Figure 6.15 - Topology optimization results by considering a involute blade as initial guess and power maximization with: (a) $20[\mathrm{rpm}]$ and (b) $100[\mathrm{rpm}]$. . 65

guess and power maximization with: (a) $20[\mathrm{rpm}]$ and (b) $100[\mathrm{rpm}]]$. . 65

Figure 6.16 - Velocity fields for turbine results by considering a involute blade as initial guess and power maximization with: (a) $20[\mathrm{rpm}]$ and (b) $100[\mathrm{rpm}] 66$

Figure 6.17 - Pressure fields for turbine results by considering a involute blade as initial guess and power maximization with: (a) $20[\mathrm{rpm}]$ and (b) $100[\mathrm{rpm}] 66$

Figure 6.18 - Entire rotor for turbine results by considering a involute blade as initial guess and power maximization with: (a) $20[\mathrm{rpm}]$ and (b) $100[\mathrm{rpm}] 67$

Figure 6.19-Rotor Model (Fluid Domain) with a Case with and without Volute . 68

Figure 6.20 - Rotor Model with a Case with and without Volute Boundary Conditions 68

Figure 6.21 - Absolute Velocity and Pressure field for Case without the volute . . . 69

Figure 6.22 - Absolute Velocity and Pressure field for Case with the volute . . . . . 69

Figure 6.23-Stream lines for both cases . . . . . . . . . . . . . . . . . . . . . . . . 69

Figure 6.24 - Pressure Field (without volute) . . . . . . . . . . . . . . . . . 70

Figure 7.1 - Case models for prototypes . . . . . . . . . . . . . . . . . 71

Figure $7.2-$ Hall sensor position . . . . . . . . . . . . . . . . . . 72

Figure $7.3-$ Motor prototype . . . . . . . . . . . . . . . . . . . . . 72

Figure 7.4 - Ring of magnets assemble . . . . . . . . . . . . . . . . . 72

Figure 7.5 - Traditional rotor prototypes . . . . . . . . . . . . . . . . . 73

Figure 7.6 - Complete pump case assemble . . . . . . . . . . . . . . . . . . . 73

Figure $7.7-3 \mathrm{D}$ printed parts . . . . . . . . . . . . . . . . . 74

Figure $7.8-$ Magnet ring and silicon steel ring built . . . . . . . . . . . . . . . 74

Figure 7.9 - Prototype assemble . . . . . . . . . . . . . . . . . 75

Figure 7.10 - Experimental setup scheme . . . . . . . . . . . . . . . . . . . 75

Figure 7.11 - Flow sensor: Flownetix 100series Smart Ultrasonic Flowmeter . . . 76

Figure 7.12 - Flow sensor experimental curve . . . . . . . . . . . . . . . . 76

Figure 7.13 - Analog manometer . . . . . . . . . . . . . . . . . . . 77

Figure 7.14 - Example of the scaling law for changes in the operating speed . . . . 79

Figure $8.1-$ Rotor post-processed design . . . . . . . . . . . . . . . . 80

Figure 8.2 - Experimental curves for straight blade rotor . . . . . . . . . . . . . 81

Figure 8.3 - Complete pump ANSYS model for the straight blade . . . . . . . . . 82

Figure $8.4-$ ANSYS result for straight blade rotor at $1815[\mathrm{rpm}]$ and $4.5[\mathrm{l} / \mathrm{min}]$. . 83

Figure 8.5 - Results comparison for Straight blade . . . . . . . . . . . . . . . 83 
Figure 8.6 - Rotors built from topology optimization results considering straight blade as initial guess and energy dissipation functional shown in Fig.

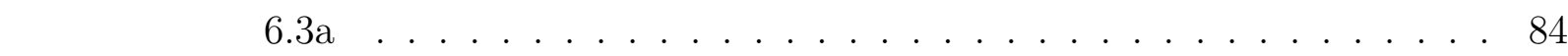

Figure 8.7 - Pump experimental curve for rotor of Fig. 8.6 . . . . . . . . . . . . . . 84

Figure 8.8 - Complete pump ANSYS model of optimized rotor for energy dissipation and straight blade as initial guess (Fig. 6.3a) . . . . . . . . . . . . . 85

Figure 8.9 - ANSYS result for optimized rotor obtained considering energy dissipation only (Fig. 6.3a), 1833[rpm] and 4.29[l/min] . . . . . . . . . . . . . . . 85

Figure 8.10 - Comparison between experimental and computational results for Optimized blade obtained by considering energy dissipation only . . . 86

Figure 8.11 - Rotors built from topology optimization results considering straight blade as initial guess and energy dissipation and vorticity functionals

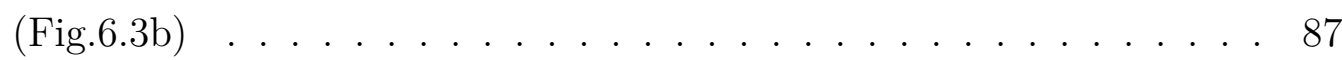

Figure 8.12 - Pump experimental curve for rotor of Fig, 8.11 . . . . . . . . . . . . . 87

Figure 8.13 - Complete pump ANSYS model of optimized rotor for energy dissipation and vorticity (Fig. 8.11] . . . . . . . . . . . . . . . 88

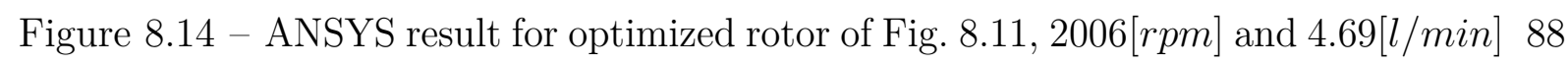
Figure 8.15 - Comparison between experimental and computational results for

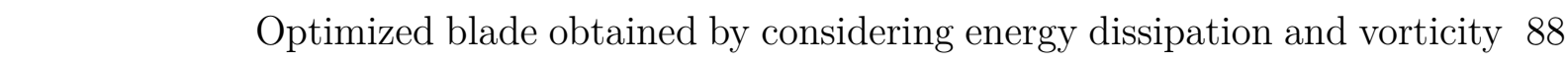
Figure 8.16 - Comparison of experimental pressure measured for the three rotors . 90 Figure 8.17 - Comparison of computational energy dissipation for the three rotors . 91 Figure 8.18 - Comparison of computational vorticity for the three rotors . . . . . . 92 Figure 8.19 - Comparison of computational torque for the three rotors . . . . . . . 94 Figure A.1 - Straight Blade: (a)Dimensions and (b)Boundary conditions . . . . . . 102 Figure A.2 - Straight Blade: Mesh, Relative velocity and Pressure fields for ANSYS and FEniCS . . . . . . . . . . . . . . . . . . . . . . . . . . . . . 104

Figure A.3 - Straight Blade: Relative velocity and Pressure fields comparison between FEniCS, ANSYS and Romero and Silva|(2014) results. . . . 105 Figure A.4 - Curved Blade: (a)Dimensions and (b)Boundary conditions . . . . . . 106 Figure A.5 - Curved Blade: Velocity and Pressure fields for ANSYS and FEniCS . 107 Figure B.1 - Wall shear stress distribution for straight blade rotor (Fig. 6.2a), $4.99[\mathrm{l} / \mathrm{min}]$ and $1920[\mathrm{rpm}]$. . . . . . . . . . . . . . . . . . . 109

Figure B.2 - Wall shear stress distribution for optimized rotor for energy dissipation, (Fig. 6.3a), 4.81[l/min] and $1923[\mathrm{rpm}]$. . . . . . . . . . . . . . . . . 109

Figure B.3 - Wall shear stress distribution for optimized rotor for energy dissipation and vorticity (Fig. 6.3b), 4.69[l/min] and $1946[\mathrm{rpm}]]$. . . . . . . . 109 


\section{LIST OF TABLES}

Table 6.1 - Functional values results by considering a straight blade as initial guess 60

Table 6.2 - Functional values results by considering a involute blade as initial guess 62

Table 6.3 - Functional values results by considering a involute blade as initial guess and power maximization . . . . . . . . . . . . . . . . . . . . 66

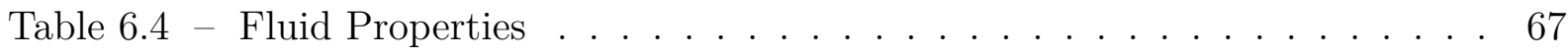

Table 8.1 - Mesh convergence of ANSYS model . . . . . . . . . . . . . . . . . 82

Table 8.2 - Functional values results for straight blade ANSYS model. . . . . . . . 83

Table 8.3 - Functional values results for the ANSYS model of optimized rotor considering straight blade as initial guess and energy dissipation only as objective function . . . . . . . . . . . . . . . 86 86

Table 8.4 - Functional values results for the ANSYS model of optimized rotor considering straight blade as initial guess and energy dissipation and vorticity. . . . . . . . . . . . . . . . . 89

Table 8.5 - Interpolated values for energy dissipation . . . . . . . . . . . . . 91

Table 8.6 - Interpolated values for vorticity . . . . . . . . . . . . . . . . . 93

Table 8.7 - Interpolated values for torque . . . . . . . . . . . . . . . . . . 94

Table A.1 - Fluid Properties . . . . . . . . . . . . . . . . . . . . . . . . 102

Table A.2 - Straight Blade Boundary Conditions . . . . . . . . . . . . . . . 103 


\section{ACRONYMS}

$\begin{array}{ll}\text { CAD } & \text { Computer Aided Design } \\ \text { CFD } & \text { Computational Fluid Dynamics } \\ \text { EDM } & \text { Electrical Discharge Machine } \\ \text { FC } & \text { Form Compiler } \\ \text { FDM } & \text { Finite Difference Method } \\ \text { FE } & \text { Finite Element } \\ \text { FEM } & \text { Finite Element Method } \\ \text { FFC } & \text { FEniCS Form Compiler } \\ \text { IPOpt } & \text { Internal Point Optimizer } \\ \text { MUMPS } & \text { MUltifrontal Massively Parallel Sparse } \\ \text { PETSc } & \text { Portable, Extensible Toolkit for Scientific Computation } \\ \text { PDE } & \text { Partial Differential Equation } \\ \text { TOM } & \text { Topology Optimisation Method } \\ \text { UFC } & \text { Unified Form-Assembly Code } \\ \text { UFL } & \text { Unified Form Language } \\ \text { WSS } & \text { Wall Shear Stress }\end{array}$




\section{NOTATION}

$\begin{array}{ll}\mathbf{u} & \text { Velocity field } \\ \mathbf{u}_{a} & \text { Absolute Velocity } \\ \mathbf{u}_{r} & \text { Relative Velocity } \\ \mathbf{u}_{t} & \text { Frame Velocity } \\ \Omega & \text { Angular Velocity } \\ \mathbf{r} & \text { Radius } \\ p & \text { Pressure field } \\ \mu & \text { Fluid viscosity } \\ \kappa & \text { Material interpolation } \\ \alpha & \text { Design variable } \\ \mathbf{\Phi} & \text { Energy dissipation } \\ J(\mathbf{u}) & \text { Vorticity } \\ \dot{W} & \text { Power } \\ \mathbf{T}_{\text {ext }} & \text { Torque } \\ w_{e}, w_{v}, w_{p} & \text { Weighting coefficients } \\ \psi & \text { Multi-objective function } \\ R e & \text { Reynolds Number } \\ \Omega & \text { Design domain } \\ u_{\text {in }} & \text { Inlet velocity } \\ p_{\text {out }} & \text { Outlet pressure } \\ u_{w a l l} & \text { Wall velocity } \\ \omega_{z} & \text { Rotation in z direction }\end{array}$




\section{CONTENTS}

$1 \quad$ INTRODUCTION $\ldots \ldots \ldots \ldots \ldots \ldots \ldots \ldots \ldots$

$1.1 \quad$ Radial Flow Machines $\ldots \ldots \ldots$

1.2 Optimization Methods $\ldots \ldots \ldots \ldots \ldots$

$1.3 \quad$ Topology Optimization Design Procedure . . . . . . . . . . . . 22

1.4 Objectives $\ldots \ldots \ldots \ldots \ldots \ldots \ldots$

1.5 Motivation . . . . . . . . . . . . . . . . . . . . . 23

1.6 Document outline . . . . . . . . . . . . . . . . . . . . . 24

2 RADIAL FLOW MACHINE MODEL $\ldots \ldots \ldots \ldots \ldots \ldots$

$2.1 \quad$ Model Description . . . . . . . . . . . . . . . 25

$2.2 \quad$ Equilibrium Equations $\ldots \ldots \ldots \ldots \ldots \ldots$

$2.2 .1 \quad$ Navier-Stokes Equations for Incompressible Fluids on Laminar Flow . . 25

$2.2 .2 \quad$ Rotating Reference System . . . . . . . . . . . . . . . . . . 26

$2.3 \quad$ Finite Element Method Applied to Flow Machines $\ldots \ldots \ldots .27$

$2.4 \quad$ Triangular Element $\ldots \ldots \ldots \ldots$

$3 \quad$ TOPOLOGY OPTIMIZATION METHOD . . . . . . . . . . . 31

$3.1 \quad$ Topology Optimization Method applied to Fluid Flow Problems . . . . 31

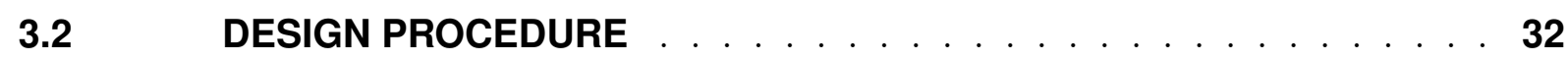

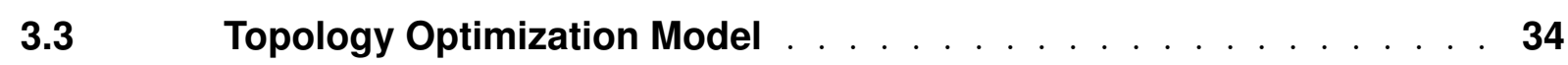

$3.3 .1 \quad$ Material Model . . . . . . . . . . . . . . . . . . . . . . . 34

$3.3 .2 \quad$ Topology Optimization Problem $\ldots \ldots \ldots \ldots$

$3.3 .3 \quad$ Multi-Objective Function . . . . . . . . . . . . . . . . . . . . 38

$3.4 \quad$ Discrete Forms of Energy dissipation, Vorticity and Power . . . . . 39

$3.4 .1 \quad$ Energy Dissipation Discrete Form . . . . . . . . . . . . . . . . . . . . 39

$3.4 .2 \quad$ Vorticity Discrete Form $\ldots \ldots \ldots \ldots$

$3.4 .3 \quad$ Power Discrete Form . . . . . . . . . . . . . . . . . . . . . . . 40

4 SENSITIVITY OF TOPOLOGY OPTIMIZATION PROBLEM $\ldots \ldots \ldots 43$

$4.1 \quad$ Adjoint Method . . . . . . . . . . . . . . . . . . . 43

$4.2 \quad$ Sensitivity Analysis by the Adjoint Method $\ldots \ldots \ldots \ldots 4$

$4.2 .1 \quad$ Energy Dissipation Sensitivity analysis . . . . . . . . . . . . . . 44

$4.2 .2 \quad$ Vorticity Sensitivity Analysis . . . . . . . . . . . . . . . . . 46

$4.2 .3 \quad$ Power Sensitivity Analysis . . . . . . . . . . . . . . . . . . . . . 47

$4.2 .4 \quad$ Multi-Objective Function Sensitivity Analysis . . . . . . . . . . . . . . 48 
5 NUMERICAL IMPLEMENTATION $\ldots \ldots \ldots \ldots \ldots \ldots \ldots$

$5.1 \quad$ Optimization Algorithm $\ldots \ldots \ldots \ldots \ldots$

5.2 Software Environment . . . . . . . . . . . . . . . . . 50

$5.2 .1 \quad$ Solving Differential Equations - The FEniCS system . . . . . . . . . . 51

$5.2 .2 \quad$ Solvers and Preconditioners . . . . . . . . . . . . . . . . . . . 51

$5.2 .3 \quad$ Solution of the Adjoint Problem . . . . . . . . . . . . . . . . 52

$5.2 .4 \quad$ Solution of the Optimization Problem . . . . . . . . . . . . 53

$5.2 .5 \quad$ Implementation of Flow Machine Rotor Optimization . . . . . . . . . . . . 54

$6 \quad$ NUMERICAL RESULTS $\ldots \ldots \ldots \ldots \ldots \ldots$

$6.1 \quad$ Topology Optimization Results $\ldots \ldots \ldots \ldots$

$6.1 .1 \quad$ Straight Blade Initial Guess . . . . . . . . . . . . . . . . . . . 57

6.1 .2 Involute Blade Initial Guess . . . . . . . . . . . . . . . . . . 60

$6.2 \quad$ Topology Results for Turbine . . . . . . . . . . . . . . . 64

$6.2 .1 \quad$ Involute Blade Initial Guess . . . . . . . . . . . . . . . . . . . . 64

$6.3 \quad$ Volute Simulation Analysis $\ldots \ldots \ldots$. . . . . . . . . 67

7 EXPERIMENTAL METHODOLOGY $\ldots \ldots \ldots \ldots \ldots \ldots$

$7.1 \quad$ Pump Prototype Design $\ldots \ldots \ldots \ldots . \ldots 71$

$7.1 .1 \quad$ Pump Case Design $\ldots \ldots \ldots \ldots 71$

$7.1 .2 \quad$ Motor Design . . . . . . . . . . . . . . . . . . . . . . . 71

$7.1 .3 \quad$ Rotor Design . . . . . . . . . . . . . . . . . . . . . . 72

$7.1 .4 \quad$ Pump Design Assemble . . . . . . . . . . . . . . . . . . . 73

$7.2 \quad$ Pump Prototype Manufacturing $\ldots \ldots \ldots \ldots \ldots$

$7.2 .1 \quad$ 3D Printed Parts . . . . . . . . . . . . . . . . . . 73

$7.2 .2 \quad$ Machined Parts . . . . . . . . . . . . . . . . . . . 74

$7.2 .3 \quad$ Prototype Assemble . . . . . . . . . . . . . . . . . . . . . . 75

$7.3 \quad$ Experimental Setup $\ldots \ldots \ldots \ldots . \ldots . \ldots 75$

$7.3 .1 \quad$ Experimental Scheme $\ldots \ldots \ldots \ldots . \ldots 75$

7.3 .2 Flow Sensor . . . . . . . . . . . . . . . . . . . . . 76

$7.3 .3 \quad$ Pressure Sensor . . . . . . . . . . . . . . . . . . . 76

$7.4 \quad$ Pump Similarity Laws $\ldots \ldots \ldots \ldots$

8 EXPERIMENTAL RESULTS $\ldots \ldots \ldots \ldots \ldots \ldots$

$8.1 \quad$ Rotor Design Interpretation $\ldots \ldots \ldots . \ldots . \ldots 8$

$8.2 \quad$ Straight Blade Experimental Result $\ldots \ldots \ldots$. . . . . . 80

$8.3 \quad$ Optimized Rotor for Energy Dissipation . . . . . . . . . . . 84

$8.4 \quad$ Optimized Rotor for Energy Dissipation and Vorticity . . . . . . . 86

$8.5 \quad$ Experimental Results Analysis . . . . . . . . . . . . . . 89

$8.5 .1 \quad$ Pressure comparison $\ldots \ldots \ldots \ldots$. . . . . . . . . . 89 
$8.5 .2 \quad$ Energy dissipation comparison $\ldots \ldots \ldots$. . . . . . . . . 90

$8.5 .3 \quad$ Vorticity comparison . . . . . . . . . . . . . . . . . . . . . . 91

$8.5 .4 \quad$ Power comparison . . . . . . . . . . . . . . . . . . 93

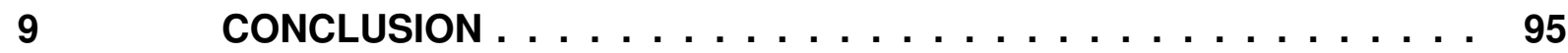

$9.1 \quad$ Future Work $\ldots \ldots \ldots$. . . . . . . . . . . . . . . . . . . 96

REFERENCES . . . . . . . . . . . . . . . . . . 97

APPENDIX A - FE RESULTS VERIFICATION . . . . . . . . . . . . . . 102

APPENDIX B - WALL SHEAR STRESS ANALYSIS $\ldots . . \ldots 108$

APPENDIX C - TECHNICAL DRAWINGS . . . . . . . . . . . . . . 110 


\section{INTRODUCTION}

\subsection{Radial Flow Machines}

Radial Flow Machines are widely spread over the industry, being used in several applications with different scales. They can be not only big sized machines, such as turbines used in thermoelectric and hydroelectric power plants, but also small sized pumps used in medical applications, such as auxiliary blood pumps (AARONSON et al., 2012).

The ventricular assist devices are temporary solutions usually being used in cases that the patient is awaiting a heart transplantation, working as a bridge between the diagnosis and the surgery (SLAUGHTER et al., 2013). The study of commercial pumps performed by Aaronson et al. (2012) shows that these pumps are becoming more and more efficient, and for patients with advanced levels of heart failure the device can be implanted, providing, in some cases, a 1-year survival, which is comparable to patients that receive the transplant.

Thus, the performance and robustness of these machines are fundamental questions for the industry, wherein small performance improvements can result not only in financial gains for big scale applications but also in an increase of life expectancy in the case of medical devices. These improvements can be done on all parts of the flow machine, such as the rotor, internal valves, bearings, nozzle and others. However, the rotor stands out for presenting a large influence on the overall performance. In an experimental work, Yu et al. (2000) estimated losses in the impeller to be about 35\% of total losses. Thus, the rotor design and evaluation of its functioning are important parts of the machine conception process.

The design and performance prediction of the rotor before its construction and implantation are difficult tasks, due to the huge number of variables involved, such as the geometry, position and number of blades. Therefore, numerical tools are used to obtain an approximation of the characteristics and performance for each rotor design JAFARZADEH et al. 2011). Thus, the performance enhancement can be obtained by a try-and-error approach, involving a number of sequential simulations in which the parameters of the rotor are manually changed at each step. However, this methodology is highly time consuming and does not imply, necessarily, in a performance optimization.

Flow machine optimization comprises from material selection to the better form and position of the blades. In particular, the blade shape optimization has been widely studied. In this process an initial shape is given and an algorithm performs local shape changes in order to improve some characteristic based on the flow around the blade (LEE et al., 2011). 
Figure 1.1 shows an example of blade flow modeling for this type of shape optimization. This blade shape optimization has, for some specific cases, analytical optimum solutions (HANSEN, 2007). However, for real problems, the boundary conditions that the blades are subjected to changes during its life cycle, so the analytical model does not work properly. Thus, a numerical optimization approach is necessary and the problem becomes a multi-objective optimization problem without analytical solution (CASAS; PENA; DURO, $2006)$.

Figure 1.1 - Radial Flow Machine Model.

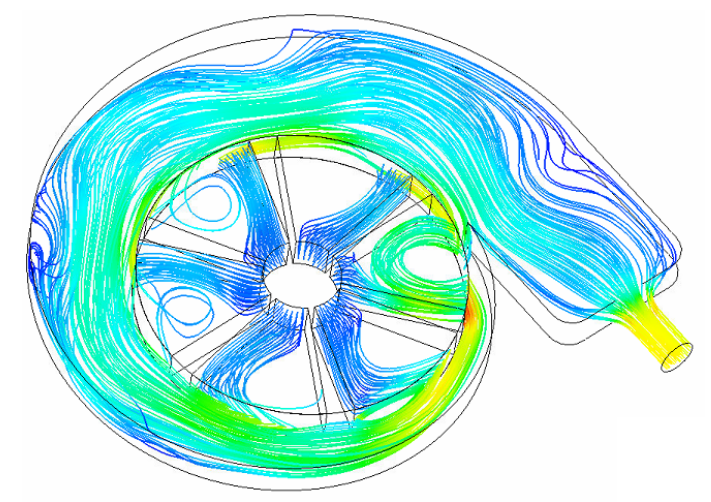

A number of works in the literature uses optimization techniques applied to these machines with different approaches, obtaining significant efficiency gains. Among them, the work of Baloni, Pathak and Channiwala (2015) performed a volute optimization based on the Taguchi Method aiming to minimize the losses inside the volute and maximize the stagnation pressure at volute outlet. They developed a 3-D numerical simulation model using the FLUENT software to simulate a centrifugal air blower. Their results indicate that for an optimum geometry, considering the desired characteristics, the volute width should be 1.5 times the impeller width. With this geometry, a higher efficiency is observed, showing an improvement of $7.4 \%$.

Considering the optimization based on the blades, the work of Wen-Guang (2011) developed a 2D blade optimization by using an inverse singularity approach applied to a curved blade design. The results are evaluated by using the CFD code Fluent and showed a $5 \%$ improvement in the hydraulic efficiency. Another work involving the blade optimization was performed by Derakhshan et al. (2013), in which a shape optimization based on the Bee Colony algorithm was applied to a centrifugal pump. They started with a curved blade geometry with arbitrary dimensions and then validated the results by using data from the Berkeh 32-160 commercial pump. Their results showed that the optimized shape is very similar to the initial shape with small curvature changes and an efficiency improvement of $3.59 \%$ was achieved.

There are other design parameters, such as the number of blades, the blade outlet angle and the impeller outlet diameter, that affect pump performance. In the case of number of 
blades the intuition suggests that a higher number of blades increases the fluid interaction and so it would promote a higher energy transmission. In fact, the pump pressure head rises as the number of blades increases. However, the presence of too many blades may cause an increase in the blockage and skin friction in the impeller passage decreasing the efficiency. However, controversially, a method to increase the efficiency is the addiction of splitters between the blades. The work of Gölcü, Pancar and Sekmen (2006) shows a study of theses splitters. They performed an analysis of impellers with 5, 6 and 7 blades, containing splitters with variable length (from $35 \%$ to $80 \%$ of the blade length) as shown in Figure 1.2. As result they achieved an increase in energy saving of $6.6 \%$ by adding splitters to an impeller with 5 blades and splitters with $80 \%$ of original blade length.

Figure 1.2 - Blade splitters.

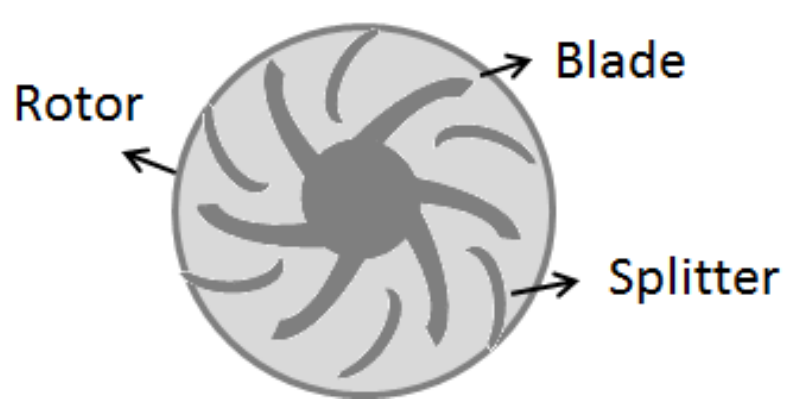

Thus, the optimization of flow machines has been widely studied over the past decades, by using different approaches and obtaining meaningful results. This work in particular focuses on applying the topology optimization method to design the rotor of radial flow machine in order to achieve non-intuitive geometries, given that this method provides more freedom to the design.

\subsection{Optimization Methods}

The optimization methods are generally divided into three categories: parametric optimization, shape optimization and topology optimization. Each one of these are better suited for a problem niche, with different constraints and objectives.

The parametric optimization is conceived as a problem where the model is parametrized in few variables, such as dimensions or rate of dimensions, geometric or material properties. This optimization allows the optimization of both system structure and process parameters. Thus, these variables become the design variables, whose values change as the optimization proceeds until an optimized solution is achieved considering the problem constraints. The work of Montevecchi et al. (1995) shows an example of optimization of a blood pump by means of parametric approach. In a topic outside the scope of this work, to illustrate the application of the parametric optimization applied to both structure and process, the work of Wang et al. (2014), in which a coal-fired power plant is analysed, can be cited. 
The shape optimization proceeds with the change of the initial domain shape. The process involves the shape boundary modification in order to better fulfill the imposed constraints. In this method, a shape that serves as a base for the algorithm is initially defined and then the algorithm modifies the domain contour during the optimization. The resulting geometry obtained has a strong dependence with the initial shape, so the pool of possible solution is restricted. Therefore, this method is better suited to refine previous optimized solutions obtained by other methods. This can be seen in the work of Derakhshan et al. (2013), where the solution obtained is very similar to the initial format.

The third category of optimization, topology optimization, uses a different approach regarding the previous techniques, in the sense that this method involves the material distribution over a specified design domain, which permits the insertion or removal of material in any point of the system, enabling more complex and non-intuitive topologies to be obtained. Thus, the resulting solution does not have a high dependency on the initial configuration, promoting greater freedom for the final topology. Therefore, structures with a better performance and more diverse topologies can be reached.

The three approaches of optimization cited above have already been applied to fluid domains, for example, to obtain flow channels with less pressure losses, as it can be seen in Figure 1.3. The first case, illustrating the parametric optimization, shows that initially the fluid enters in one point and flows through all pipes until the outlet and the optimization is performed by selecting the minimum number of pipes that connect the entrance point to the outlets. The second case shows the shape optimization applied to a diffuser, in which the straight contour is changed to a spline curve. The third case shows the topology optimization to design the optimum geometry connecting the two inlets and the two outlets.

Figure 1.3 - Three optimization approaches applied to design of a flow channel by considering minimization of pressure loss.

Parametric Optimization
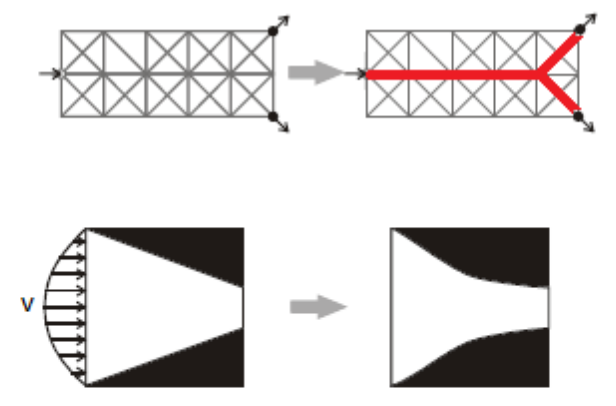

Topology Optimization

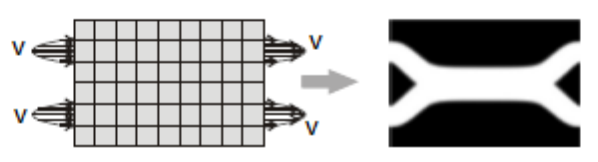

Adapted from Koga 2010)
Tube elements

\author{
Spline Curves
}

Material Distribution 


\subsection{Topology Optimization Design Procedure}

The traditional approach to topology optimization consists of a combination of an analysis method, such as the Finite Element Method (FEM), and a numerical optimization algorithm. In the topology optimization method, a design variable is defined over a domain $(\Omega)$, which is modified as the algorithm proceeds.

Initially a design domain is defined, then a discretization technique is used to produce a mesh, dividing the domain in elements. In principle any element and mesh geometry can be used in order to better fulfill the project requirements. When the elements are chosen, the nodes and degrees of freedom are defined.

The next step is the definition of the boundary conditions. These are conditions that specify values to the problem variables (Dirichlet Conditions), such as walls with null velocity, or to derivatives of problem variables (Neumann Conditions).

The optimization procedure initiates by defining the objective function based on the desired characteristics. Then, the finite element problem is solved, considering the defined mesh and boundary conditions. This gives the velocity and pressure distribution over the domain. Then, the objective function is evaluated, and the gradient of the objective function and of the constraints with respect to the design variables are calculated. Other constraints, such as the volume constraint, are also verified. The optimization algorithm modifies the design variable following the gradient information, iteratively, to reach an optimized material distribution. The iterative process goes on until the convergence criteria is met.

After the process converges the optimum topology is post-processed, in order to smooth the contour, and it is re-evaluated to verify if the constraints are respected. Finally, the design can be built.

Figure 1.4 illustrates this optimization procedure applied to fluid domains. The initial domain and the velocity boundary condition at the inlet and at the outlet are defined. Then, the domain is discretized producing the mesh with the desired elements. At the discretization, step the degrees of freedom and variables of the problem are defined, such as velocity and pressure variables at each node of the mesh. The optimized topology is reached after changing the domain topology accordingly to the gradient of the objective function. The final geometry has to be post-processed to smooth its contour. The verification step is necessary to evaluate that the desired objective is reached even with the post-processed modifications. Finally, the optimized geometry can be built. 
Figure 1.4 - Topology optimization method procedure for fluid domains.
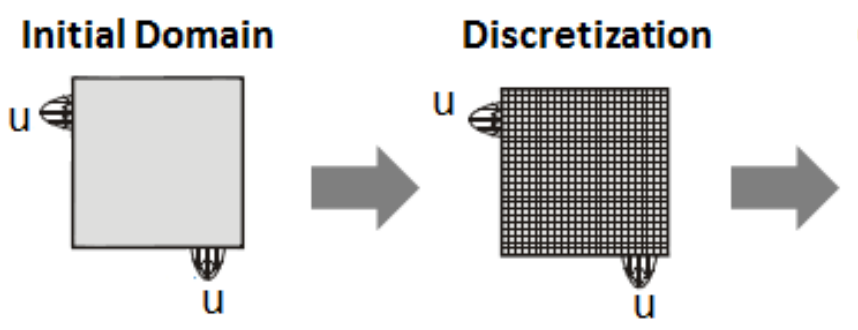

\section{Optimum Topology}

Prototype
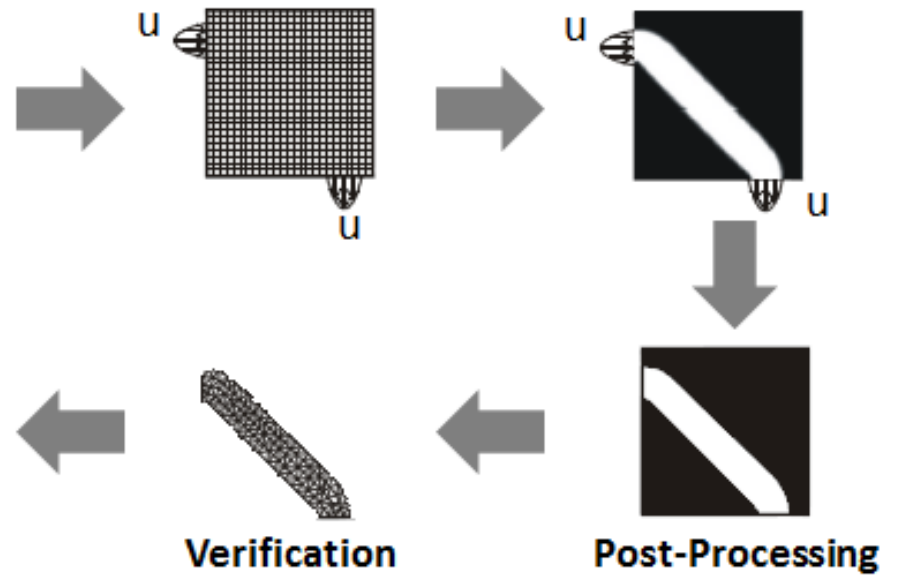

Adapted from Koga (2010)

\subsection{Objectives}

This works aims to develop a methodology for the optimum design of flow machines rotors by using the topology optimization method. Thus, it involves the creation of a process to get through all the steps of the design, passing by listing the design requisites, developing simulation models, implementing topology optimization design, post-processing and finally, building a prototype and testing it. In order to illustrate the methodology it is applied to the development of a small scale pump representing a ventricular assist device. Thus, the design requisites chosen are minimization of energy dissipation, vorticity and power. The vorticity is specially chosen to emulate the minimization of the shear stress at the walls, given that the shear stress is one of the main causes of hemolysis. The formulation is implemented based on the work of Romero and Silva (2014). The algorithm is implemented using the FEniCS platform to solve the finite element analysis and the topology optimization is executed by the adjoint method in association with the PyIPOpt optimizer. The results obtained are validated in the ANSYS software. The final topologies are post-processed and CAD models of the rotors are created by using the Autodesk Inventor software. Finally, prototypes are built using a 3D printer and then a characterization is performed by measuring the mass flow and pressure at the pump outlet.

\subsection{Motivation}

The design of generic flow machines is complex, due to the fact that each application has its own requisites, for example, the ventricular auxiliary cardiac pump depends on a low vorticity, while, industrial applications demand for high energy efficiency (low energy 
dissipation). Thus, the systematic design process can be facilitated by using topology optimization algorithm, enabling to develop machines with different requisites, lowering the development time of machines for specific applications. Besides, the application of the topology optimization method promotes a higher flexibility for obtaining non-intuitive geometries.

\subsection{Document outline}

This document is organized as follows. In Chapter 2, the formulation used to model radial flow machine is described and the finite element model for solving the equations by using the weak-formulation approach is introduced. Chapter 3 presents the proposed methodology, the optimization technique and the desired objective functions, also applying the radial flow machine model to the optimization equations. In Chapter 4 , the adjoint method is described in order to perform the sensitivity analysis, showing the adjoint models for each objective function. Chapter 5 presents the numerical implementation, introduces the software framework and the solvers used and describes the libraries in which the developed algorithm relies on. Chapter 6 presents an analysis of the volute influence and the resulting topologies obtained through the topology optimization method. Chapter 7 shows the experimental setup and the prototypes built in order to validate the design process. Chapter 8 shows the experimental results obtained by testing the prototypes. Finally, Chapter 9 reports overall conclusions on the results and suggestions for future works. The verification of the implemented algorithm, an analysis of the wall shear stress and the technical drawings are presented in the Appendix. 


\section{RADIAL FLOW MACHINE MODEL}

\subsection{Model Description}

The flow inside radial flow machines can be modeled as a fluid flow in a straight channel that rotates around a fixed-frame axis (inertial frame), as shown in Figure 2.1. Thus, the Navier-Stokes equations are used with the addition of body forces representing the rotation terms imposed by the rotor. These terms are relative to the fluid inertia, introducing terms relatives to Coriolis and Centrifugal forces. Since these models are more complex they require numerical methods, such as the Finite Element Method (FEM), to be solved (ZIENKIEWICZ; TAYLOR; ZHU, 2013). The system of partial differential equations and the FE modeling are described in the Section 2.2.1.

This work only considers newtonian fluids in the modeling. The non-newtonian model admits a model for the viscosity, i.e., the viscosity is no longer constant and follow certain equations. However, the behavior of the non-newtonian fluids are expected to be very similar to the newtonian fluids behavior in high Reynolds.

Figure 2.1 - Rotor Model approximation by using a Rotational Straight Channel Flow.

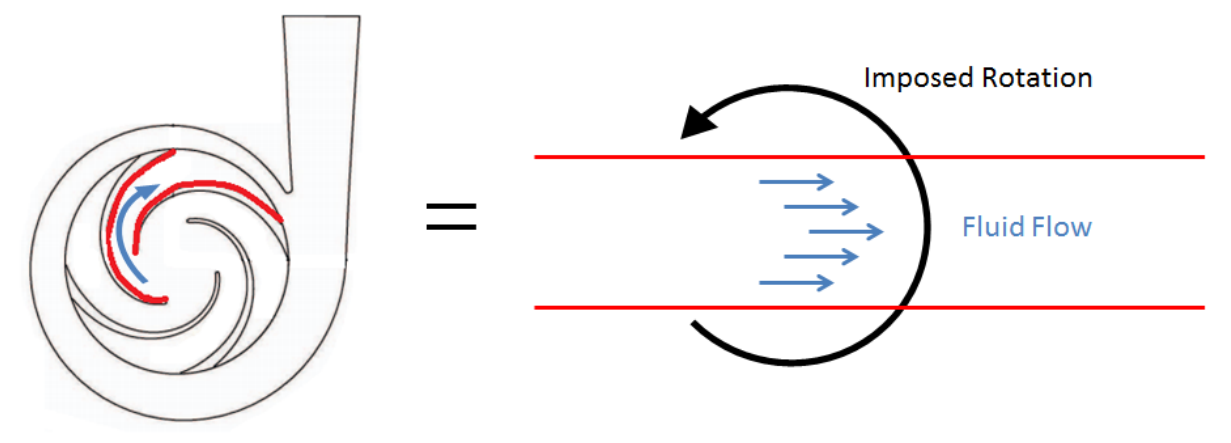

\subsection{Equilibrium Equations}

\subsubsection{Navier-Stokes Equations for Incompressible Fluids on Laminar Flow}

A first approach for modeling the flow machine will assume newtonian flows and low Reynolds numbers will be assumed and only steady state analysis will be considered. Thus, incompressible Navier-Stokes equations will be used (WHITE, 2010), which are given by:

$$
\rho \frac{D \mathbf{u}}{D t}=-\nabla p+\mu \nabla \cdot\left(\nabla \mathbf{u}+\nabla \mathbf{u}^{T}\right)+\rho \mathbf{b}
$$

where $\mathbf{u}$ is the velocity field, $p$ is the pressure, $\mu$ is the viscosity and $\rho$ is the mass density. 
The material derivative operator is defined by $\frac{D \mathbf{u}}{D t}=\frac{\partial \mathbf{u}}{\partial t}+\nabla \mathbf{u} \cdot \mathbf{u}$, where $\nabla \mathbf{u}=\frac{\partial u_{i}}{\partial x_{j}} \mathbf{e}_{i} \mathbf{e}_{j}$ and, due to steady states consideration, $\frac{\partial \mathbf{u}}{\partial t}=0$. The term $\mathbf{b}$ represent the body forces.

The incompressibility $(\nabla \rho=0)$ imposed simplifies mass conservation equation to:

$$
\nabla \cdot \mathbf{u}=\mathbf{0}
$$

\subsubsection{Rotating Reference System}

Modeling a radial flow machine, as shown in Figure 1.1, involves manipulating rotating reference systems, so it is necessary to find a relation between flow motion equations and this rotating reference. Defining $\mathbf{u}_{a}$ as the absolute velocity field, $\mathbf{u}_{r}$ as the relative velocity field of the rotating system, and $\mathbf{u}_{t}=\boldsymbol{\omega} \times \mathbf{r}$ as the frame velocity, we obtain (KUNDU; COHEN; DOWLING, 2013):

$$
\mathbf{u}_{a}=\mathbf{u}_{r}+\mathbf{u}_{t}=\mathbf{u}_{r}+\boldsymbol{\omega} \times \mathbf{r}
$$

By introducing a resistive force $\mathbf{f}_{r}$ representing the porosity, Equation 2.1 can be rewritten as:

$$
\rho \nabla \mathbf{u}_{r} \cdot \mathbf{u}_{r}=-\nabla p+\mu \nabla \cdot\left(\nabla \mathbf{u}_{r}+\nabla \mathbf{u}_{r}^{T}\right)+\rho \boldsymbol{f}-2 \rho \boldsymbol{\omega} \times \mathbf{u}_{r}-\rho \boldsymbol{\omega} \times \boldsymbol{\omega} \times \mathbf{r}+\mathbf{f}_{r}
$$

where $\mathbf{f}_{r}$ is the fluid viscous resistance, that arises when considering flow through a porous media due to friction of fluid on the solid structure. It is assumed that this resistance is proportional to the relative velocity between the fluid and fixed region, and it is given by $\mathbf{f}_{r}=-\kappa(\alpha) \mathbf{u}_{r}$ (Darcy's Law), which is defined over the entire domain.

Two terms stand out on Equation 2.4, these terms arise due to system rotation representing Coriolis force $\left(\mathbf{f}_{c o}\right)$ and Centrifugal force $\left(\mathbf{f}_{c e}\right)$, as shown on the following equations:

$$
\begin{array}{lr}
\mathbf{f}_{c o}=-2 \rho \boldsymbol{\omega} \times \mathbf{u}_{r} & (\text { Coriolis) } \\
\mathbf{f}_{c e}=-\rho \boldsymbol{\omega} \times \boldsymbol{\omega} \times \mathbf{r} & \text { (Centrifugal) }
\end{array}
$$

Finally, by introducing the term $\kappa(\alpha)$ representing domain porosity into the Navier-Stokes steady state equation with rotational domain (2.4), the Brinkman model is obtained with a convection term (BORRVALL; PETERSSON, 2003), given by:

$$
\rho \nabla \mathbf{u}_{r} \cdot \mathbf{u}_{r}=\nabla \cdot \mathbf{T}+\rho \mathbf{f}-2 \rho \boldsymbol{\omega} \times \mathbf{u}_{r}-\rho \boldsymbol{\omega} \times \boldsymbol{\omega} \times \mathbf{r}-\kappa(\alpha) \mathbf{u}_{r}
$$

where $\mathbf{T}=-p \mathbf{I}+\tau$ is the stress tensor, $\tau$ is the viscous stress tensor, given by $\tau=$ $\mu\left(\nabla \mathbf{u}_{r}+\nabla \mathbf{u}_{r}^{T}\right)$. The $\tau$ tensor can be represented as a function of relative velocity, since 
the angular velocity has no effect on the internal forces of the fluid. Given those internal forces, by definition, cannot be influenced by the relative motion of reference systems (KUNDU; COHEN; DOWLING, 2013).

\subsection{Finite Element Method Applied to Flow Machines}

The partial differential equations (PDE) shown on the previous section are hard to solve analytically. However, it is possible to use a numerical approach in order to obtain an approximate solution that is subjected to precision criteria and computational cost. Hence, it is plausible to use a solution method such as the Finite Element Method (FEM) or the Finite Volume Method (FVM). It is usual for commercial software to use the FVM, however in this work the FEM is chosen, given that it is more often seen in the literature with topology optimization applications.

To solve the system of equations, the weighted residuals procedure of Galerkin's method is applied to the weak formulation of a Navier-Stokes problem in a rotating reference system.

A weighted residual is obtained by multiplying the original PDE by a test function and then integrating the inner product over the domain. Using the Galerkin's method means that the unknown field is represented by the same function space used for the weight functions.

The weak formulation for the complete problem, involving Navier-Stokes equations together with a porous media flow, in a rotating reference system considering the steady state is given by (ROMERO; SILVA, 2014):

$$
\begin{gathered}
R_{c}=\int_{\Omega}(\nabla \cdot \mathbf{u}) M d \Omega=0 \\
R_{m}=\int_{\Omega}(\rho \mathbf{u} \nabla \mathbf{u}) \cdot \mathbf{W} d \Omega+\int_{\Omega} \kappa(\mathbf{x}) \mathbf{W} \cdot \mathbf{u} d \Omega+\int_{\Omega} \mathbf{T}: \nabla \mathbf{W} d \Omega \\
+\int_{\Omega} \mathbf{W}[2 \rho(\boldsymbol{\omega} \times \mathbf{u})+\rho \boldsymbol{\omega} \times(\boldsymbol{\omega} \times \mathbf{r})] d \Omega-\int_{\Omega} \mathbf{b} \cdot \mathbf{W} d \Omega-\int_{\Gamma}(\mathbf{T} \cdot \mathbf{n}) \cdot \mathbf{W} d \Gamma=0,
\end{gathered}
$$

where $\mathbf{W}$ and $M$ are the test functions, $M$ is scalar, $\mathbf{W}$ is a vector and $\mathbf{b}$ represents the external body forces, such as gravity. The Galerkin's method is applied choosing the "test" functions as the weighting functions, so $\mathbf{W}=\Phi_{i}(\mathbf{x})$ and $M=\chi_{i}(\mathbf{x})$. The weighting function $\Phi_{i}$ associated with the momentum equations is bi-quadratic and $\chi_{i}$ associated to continuity equation is linear. 
The stress tensor $\mathbf{T}$ is given by:

$$
\mathbf{T}=\left[\begin{array}{cc}
-p+2 \mu \frac{\partial u_{1}}{\partial x_{1}} & \mu\left(\frac{\partial u_{1}}{\partial x_{2}}+\frac{\partial u_{2}}{\partial x_{1}}\right) \\
\mu\left(\frac{\partial u_{1}}{\partial x_{2}}+\frac{\partial u_{2}}{\partial x_{1}}\right) & -p+2 \mu \frac{\partial u_{2}}{\partial x_{2}}
\end{array}\right]
$$

Reynolds number is defined as $R e=\rho U R / \mu$, where $U$ is a reference velocity $\left(U=\Omega_{0} R\right)$, $\Omega_{0}$ is the angular velocity, and $R$ is a length scale (here the impeller radius) (ROMERO; SILVA, 2014).

The finite element method is implemented by using Taylor-Hood elements, where the fluid velocity and pressure have different interpolation ranks. In this case, the velocity has a quadratic interpolation and pressure has a linear interpolation, as shown in Figure 2.2. This is done to avoid stability issues in the solution, given that this element interpolation combination addresses the "Ladyzhenskaya-Babuska-Brezzi (LBB) condition". The design variable is defined at the center of the element.

Considering a triangular element, the velocity field $\mathbf{u}=\left[u_{1} u_{2}\right]^{T}$ and pressure $p$ are approximated by a linear combination of the basis functions in the form

$$
u_{1}(\mathbf{x})=\sum_{j=1}^{n} \phi_{j}(\mathbf{x}) u_{j}^{1}=\mathbf{\Psi}^{T} \mathbf{u}_{1} ; u_{2}(\mathbf{x})=\sum_{j=1}^{n} \phi_{j}(\mathbf{x}) u_{j}^{2}=\Psi^{T} \mathbf{u}_{2} ; p(\mathbf{x})=\sum_{j=1}^{m} \chi_{j}(\mathbf{x}) p_{j}=\chi^{T} \mathbf{p}
$$

where $\mathbf{u}_{1}, \mathbf{u}_{2}$ and $\mathbf{p}$ are vectors with the nodal values of the approximated solution for the velocity field and pressure, defined in the weak formulation of the finite element method. The vectors $\boldsymbol{\Psi}$ and $\boldsymbol{\chi}$ represent the interpolation functions of the triangular element and are defined in the next section.

Replacing these approximations (Eq. 2.11) in equations 2.8 and 2.9, the algebraic equations of the finite element method are obtained. For the two-dimensional case the system is given by:

$$
\begin{gathered}
{\left[\begin{array}{ccc}
\mathbf{K}_{k} & 0 & 0 \\
0 & \mathbf{K}_{k} & 0 \\
0 & 0 & 0
\end{array}\right]\left\{\begin{array}{l}
\mathbf{u}_{1} \\
\mathbf{u}_{2} \\
\mathbf{p}
\end{array}\right\}+\left[\begin{array}{ccc}
\mathbf{C}_{1}\left(\mathbf{u}_{1}\right)+\mathbf{C}_{2}\left(\mathbf{u}_{2}\right) & 0 & 0 \\
0 & \mathbf{C}_{1}\left(\mathbf{u}_{1}\right)+\mathbf{C}_{2}\left(\mathbf{u}_{2}\right) & 0 \\
0 & 0 & 0
\end{array}\right]\left\{\begin{array}{l}
\mathbf{u}_{1} \\
\mathbf{u}_{2} \\
\mathbf{p}
\end{array}\right\}} \\
+\left[\begin{array}{ccc}
2 \mathbf{K}_{11}+\mathbf{K}_{22} & \mathbf{K}_{12} & -\mathbf{Q}_{1} \\
\mathbf{K}_{21} & \mathbf{K}_{11}+2 \mathbf{K}_{22} & -\mathbf{Q}_{2} \\
-\mathbf{Q}_{1}^{T} & -\mathbf{Q}_{2}^{T} & 0
\end{array}\right]\left\{\begin{array}{l}
\mathbf{u}_{1} \\
\mathbf{u}_{2} \\
\mathbf{p}
\end{array}\right\}+\left[\begin{array}{ccc}
0 & \mathbf{K}_{r} & 0 \\
-\mathbf{K}_{r} & 0 & 0 \\
0 & 0 & 0
\end{array}\right]\left\{\begin{array}{l}
\mathbf{u}_{1} \\
\mathbf{u}_{2} \\
\mathbf{p}
\end{array}\right\}=\left\{\begin{array}{l}
\mathbf{f}_{1} \\
\mathbf{f}_{2} \\
\mathbf{0}
\end{array}\right\}
\end{gathered}
$$


and the matrices coefficients are defined as

$$
\begin{aligned}
\mathbf{K}_{\kappa} & =\int_{\Omega^{e}} \kappa \boldsymbol{\Psi} \boldsymbol{\Psi}^{T} d x \\
\mathbf{C}_{j}\left(\mathbf{u}_{j}\right) & =\int_{\Omega^{e}} \rho_{0} \boldsymbol{\Psi}\left(\boldsymbol{\Psi}^{T} \mathbf{u}_{i}\right) \frac{\partial \boldsymbol{\Psi}}{\partial x_{j}} d x \\
\mathbf{K}_{i j} & =\int_{\Omega^{e}} \mu \frac{\partial \boldsymbol{\Psi}}{\partial x_{j}} \frac{\partial \boldsymbol{\Psi}^{T}}{\partial x_{i}} d x ; \\
\mathbf{Q}_{i} & =\int_{\Omega^{e}} \frac{\partial \boldsymbol{\Psi}}{\partial x_{i}} \chi^{T} d x \\
\mathbf{K}_{r} & =\int_{\Omega^{e}} 2 \Omega_{0} \boldsymbol{\Psi} \boldsymbol{\Psi}^{T} d x ; \\
\mathbf{f}_{i} & =\int_{\Omega^{e}} \rho \boldsymbol{\Psi} f_{i} d x+\int_{\Omega^{e}} \rho \Omega_{0}^{2} \boldsymbol{\Psi} x_{i} d x+\oint_{\Gamma^{e}} h_{i} \boldsymbol{\Psi} d s, \quad i, j=1,2 ;
\end{aligned}
$$

being $\kappa=\kappa\left(\alpha_{i}\right)$. The matrix $\mathbf{K}_{\kappa}$ can also be defined as

$$
\mathbf{K}_{\kappa}=\kappa \mathbf{K}_{m} \quad \text { where, } \mathbf{K}_{m}=\int_{\Omega^{e}} \boldsymbol{\Psi} \Psi^{T} d x
$$

The resulting system is non-linear due to the term $\mathbf{C}(\mathbf{u})$, which is non-linear and non-symmetrical. Therefore, to solve the system an non-linear solver needs to be applied, generally based on Newton method. The velocity and pressure fields are the state variables of the problem, and the residual can be defined in a compact form based on Equation 2.12 as

$$
\mathbf{r}(\mathbf{z}(\kappa), \kappa)=0
$$

where $\mathbf{z}$ is a vector containing all state variables, $\mathbf{z}=\left[\mathbf{u}_{1} \mathbf{u}_{2} \mathbf{p}\right]^{T}$, and it is shown that $\mathbf{r}$ and the state variables explicitly depend on the material model $\kappa$.

\subsection{Triangular Element}

The Finite Element Method uses an interpolation function to approximate the variables inside the elements by using their nodal values. In this work, a triangular element is used with different polynomial interpolation ranks. This is done in order to avoid stability issues in the solution. As stated before, the Taylor-Hood element is used, in which the pressure uses a triangular element with three nodes (linear interpolation) and the velocity uses a triangular element with six nodes (quadratic interpolation). The inverse permeability $(\kappa(\alpha))$ uses one node per element, as shown on Figure 2.2 .

The interpolation inside the element is done in a local coordinate frame (Fig. 2.3), defined by $\xi$ and $\eta\left(\Omega_{\text {standard }} \in[0,1] \times[0,1]\right)$ and then transformed to the global coordinate frame by using the following relation, for a triangular element with vertices $\left(x_{1}, y_{1}\right),\left(x_{2}, y_{2}\right)$ 
Figure 2.2 - Triangular Element Interpolation: (a)Pressure, (b)Velocity and (c)Design variable.

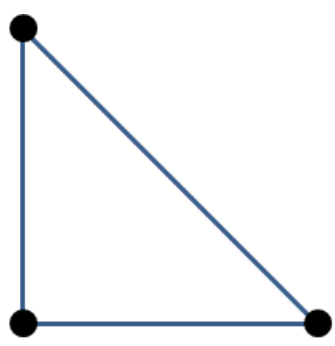

(a) Pressure Interpolation

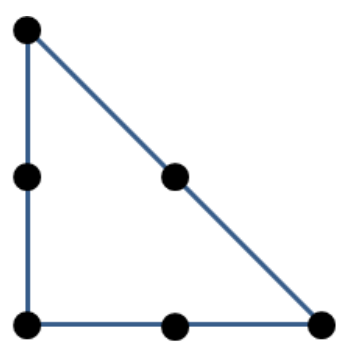

(b) Velocity Interpolation

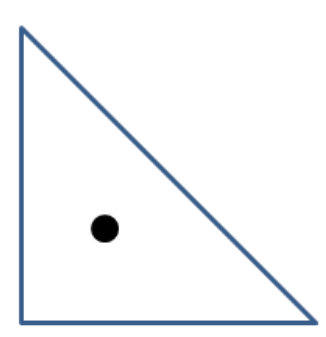

(c) Design Variable Interpolation

and $\left(x_{3}, y_{3}\right)$ :

$$
\begin{aligned}
& x=x_{1}+\left(x_{2}-x_{1}\right) \xi+\left(x_{3}-x_{1}\right) \eta \\
& y=y_{1}+\left(y_{2}-y_{1}\right) \xi+\left(y_{3}-y_{1}\right) \eta
\end{aligned}
$$

Figure 2.3 - Triangular element local coordinate frame.

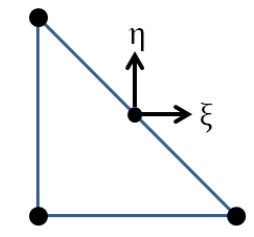

The interpolation for the continuous pressure distribution in the element, using the local coordinates in Equation 2.11, is given by:

$$
\begin{aligned}
& \chi_{1}(\xi, \eta)=1-\xi-\eta \\
& \chi_{2}(\xi, \eta)=\xi \\
& \chi_{3}(\xi, \eta)=\eta
\end{aligned}
$$

The velocity is interpolated quadratically, so using the local coordinates in Equation 2.11, it gives:

$$
\begin{aligned}
& \Psi_{1}(\xi, \eta)=(1-\xi-\eta)(1-2 \xi-2 \eta) \\
& \Psi_{2}(\xi, \eta)=\xi(2 \xi-1) \\
& \Psi_{3}(\xi, \eta)=\eta(2 \eta-1) \\
& \Psi_{4}(\xi, \eta)=4 \xi(1-\xi-\eta) \\
& \Psi_{5}(\xi, \eta)=4 \xi \eta \\
& \Psi_{6}(\xi, \eta)=4 \eta(1-\xi-\eta)
\end{aligned}
$$




\section{TOPOLOGY OPTIMIZATION METHOD}

\subsection{Topology Optimization Method applied to Fluid Flow Problems}

In the topology optimization method material is distributed (fluid or solid) over a domain, aiming to maximize (or minimize) an objective function under determined constraints. This method was firstly introduced to fluid domains by Borrvall and Petersson (2003), where they apply the method to 2D flow channel problems, aiming to minimize the energy dissipation over the domain. In that case, the flow was modelled by using the Stokes equations for incompressible flows, without considering body forces, and with low Reynolds numbers. One of their results is shown in Figure 3.1, where the resulting topologies considering a channel with two inlets on the left side and two outlets on the right side and different aspect ratios are presented.

Figure 3.1 - Topology optimization applied to flow channel problem.
(a) Aspect ratio $1 \times 1$.
(b) Aspect ratio $1 \times 1.5$.
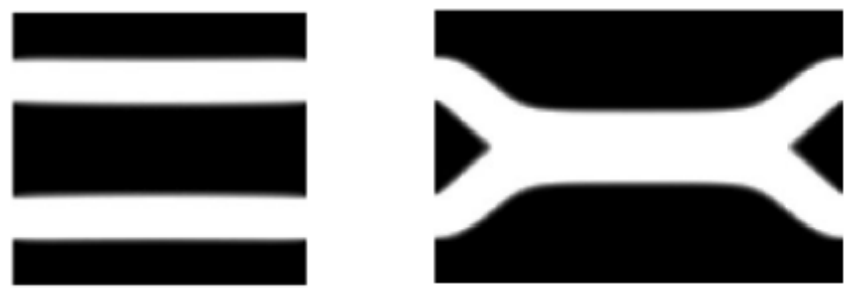

Adapted from (BORRVALL; PETERSSON, 2003)

Gersborg-Hansen (2003), Gersborg-Hansen, Sigmund and Haber (2005) and Olesen, Okkels and Bruus (2006) extended this approach by using Navier-Stokes equations for a wider Reynolds number range and considering additional effects, such as body force and non-linearities. Besides that, the work of Gersborg-Hansen also investigated different objective functions, other than energy dissipation, so with their implementation it is possible to design systems based on velocity variation across the channel, such as directional flow selectors, in which the path chosen by the fluid depends on its velocity.

Evgrafov (2005) reassesses the work of Borrvall and Petersson (2003) and compares the Brinkman model used with a different approach, by considering the fluid viscosity as a problem variable. Evgrafov also studied the application of topology optimization to slightly compressible fluid (EVGRAFOV, 2004). The effects of non-newtonian fluids over the topology optimization was evaluated by Pingen and Maute (2010).

Another approach to fluid optimization was developed by Wiker, Klarbring and Borrvall (2007), in which a specific region optimization separates the distribution of material under 
Stokes flow and porous media (Darcy flow). Thus, the resulting topology has regions where only fluid is present (Stokes flow) and regions where a porous media is established.

In the work of Deng et al. (2013) the topology optimization method is applied to the flow channel problem, aiming to minimize the pressure loss, considering Navier-Stokes formulation and the presence of body forces. They solve the system problem with the adjoint method and the software is implemented by using the commercial software COMSOL Multiphysics.

The topology optimization method applied to flow machines has been recently studied in the work of Romero and Silva (2014), in which the fluid flow in the machine is modeled as Navier-Stokes flow with the addition of a rotating reference system, arising the effects of Centrifugal force and Coriolis force. In their work various configurations are proposed for the machine rotor, exploring the influence of the initial domain and the effects of changes in the boundary conditions. As a result non-intuitive geometries, which differs from traditional ones, are obtained, for example the topology shown in Figure 3.2. Thus, the present work has its bases on their work (ROMERO; SILVA, 2014) and uses a very similar methodology.

Figure 3.2 - Topology optimization method applied to design of a straight blade rotor by considering energy dissipation as objective function.

(b) Resulting Topology for

(a) Initial Straight Blade. Straight Blade initial

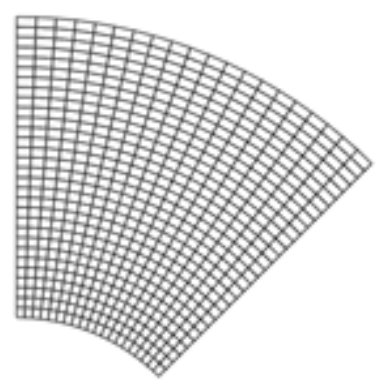
domain.

Adapted from (ROMERO; SILVA, 2014)

\subsection{DESIGN PROCEDURE}

This work aims to develop a methodology to design radial flow machine rotors. This chapter explains the overall process and the actions to be taken in order to successfully complete the steps.

The method initiates with the requisite definition phase, where a professional that has sufficient knowledge of the application identifies the operational requisites, such as the geometric dimensions, the mass flow and the pressure. Also the characteristics that are 
important to be optimized are defined. For example, the ventricular assist device has a typical operational requisite of mass flow of $5[\mathrm{l} / \mathrm{min}]$ and pressure of $120[\mathrm{~mm} \mathrm{Hg}]$, and the desired characteristic of minimizing the vorticity, that has a correlation with the wall shear stress and consequently with hemolysis rate.

The operational requisites are used to define the boundary conditions for the finite element model. The mass flow $(Q)$ and the inlet dimensions (area $A$ ) define the inlet velocity $\left(u_{i n}\right)$, given that $Q=A \cdot u_{i n}$. The pressure at the model outlet is defined zero and the operational pressure (measured) is compared to the simulated pressure to verify the model.

The objective functions are defined in order to optimize the design given certain conditions. Thus, in the case of a multi-objective function, the weighting coefficients among the different functions are defined by an engineer and they are selected to prioritize an objective over another. In this work, three functions are defined: the energy dissipation function, the vorticity function and the power function. Also, three coefficients are defined representing each function: $w_{e}, w_{v}$ and $w_{t}$. Hence, the examples explore the variation of the weighting coefficients among the three functions, considering that the sum of them is equal to $1\left(w_{e}+w_{v}+w_{t}=1\right)$.

The second phase includes solving the FEM system and the topology optimization in order to obtain the optimized designs.

The third phase is composed of the post-processing since the resulting blade needs to be isolated from the domain and the results are obtained with arbitrary dimensions. Thus, a model re-scaling is needed before the CAD software designing. The final contour is smoothed using spline curves in the CAD process. Then. they are reproduced in a circular pattern in a way that maximizes the number of blades without overlap. This phase can be seen in Figure 3.3 .

The fourth phase involves building and testing the prototypes. The final design is exported in a file format that the $3 \mathrm{D}$ printer accepts, in this case the STL format (STereoLithography), and it is printed. Then, the prototype is tested by measuring the pressure head and the mass flow at the pump outlet.

The following chapters describe how to proceed in each phase. 
Figure 3.3 - Complete cycle for development of a flow machine rotor by using topology optimization.

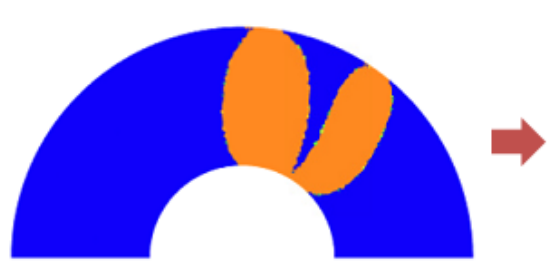

FEniCS result

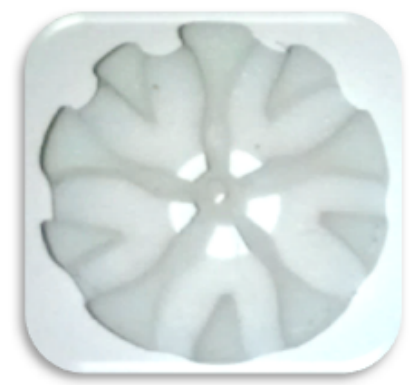

3D printed rotor

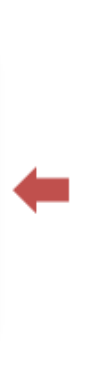

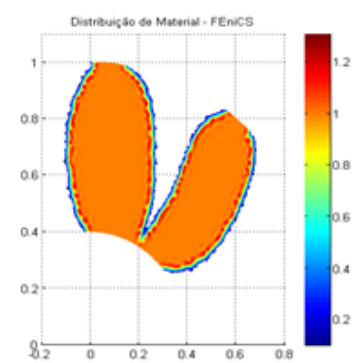

Isolated blade (Matlab)

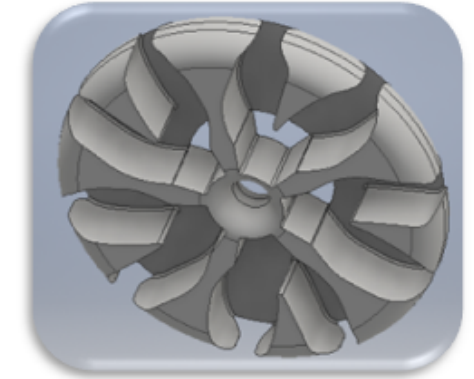

3D model (CAD)

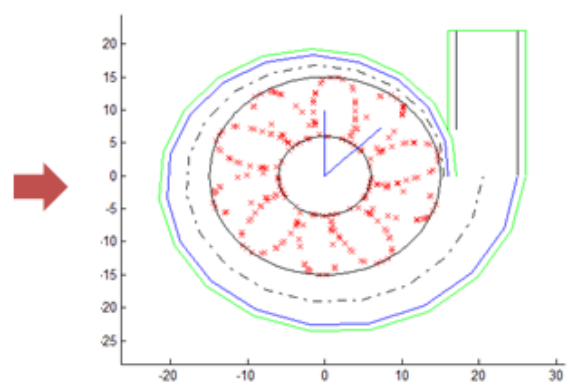

Re-scale and produce the rotor (Matlab)

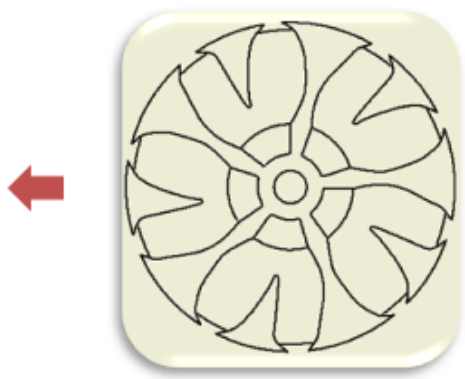

Smooth with splines (CAD)

\subsection{Topology Optimization Model}

\subsubsection{Material Model}

The topology optimization method involves distributing material over a specified domain by following certain requisites. Therefore, in order to determine the path to be followed by the fluid flow and to define a design variable to perform a topology optimization, a porous domain was introduced by Borrvall and Petersson (2003), dividing domain regions between high permeability material, interpreted as pure fluid, and low permeability material, representing solid, i.e., the porosity virtually separates fluid and solid regions (GERSBORG-HANSEN, 2003). This is done with the introduction of the absorption term $\kappa$ that controls the material distribution in the domain.

The absorption coefficient $\kappa$ can be considered as a interpolation of the material flux on the domain, which represents the transition of material between high porosity (solid, $\kappa \gg 1$ ) and low porosity (fluid, $\kappa=0$ ) regions. This coefficient is a function of $\alpha$, a pseudo-density field which is the optimization problem design variable. The choice of this function $\alpha \rightarrow \kappa(\alpha)$ makes the design variable to assume intermediates values, different from 0 or 1 . So, to suppress these undesired values a convex and q-parameterized interpolation 
function can be chosen (BORRVALL; PETERSSON, 2003):

$$
\kappa(\alpha)=\kappa_{\max }+\left(\kappa_{\min }-\kappa_{\max }\right) \alpha \frac{1+q}{\alpha+q}
$$

with $\kappa \in\left[\kappa_{\min }, \kappa_{\max }\right], \alpha$ represents points inside the domain and $q$ is a parameter that controls $\kappa$ linearity. Thus, for $q \rightarrow \inf , \kappa \rightarrow \kappa_{\max }+\left(\kappa_{\min }-\kappa_{\max }\right) \alpha$ is a linear function. Figure 3.4 illustrates this behavior by considering $\kappa_{\max }=10000$ and $\kappa_{\min }=0$. The optimization process aims to obtain values of 0 or 1 to the design variable $\alpha(\alpha \approx 0$ or $\alpha \approx 1$ ), because a intermediate value would not have a physical meaning. Therefore, when $\alpha \approx 1 \Rightarrow \kappa=\kappa_{\min }$, represents a flow of pure fluid, while when $\alpha \approx 0 \Rightarrow \kappa=\kappa_{\max }$, represents a restricted flow inside a porous media.

Figure 3.4 $-\kappa$ as function of parameters $\alpha$ and q.

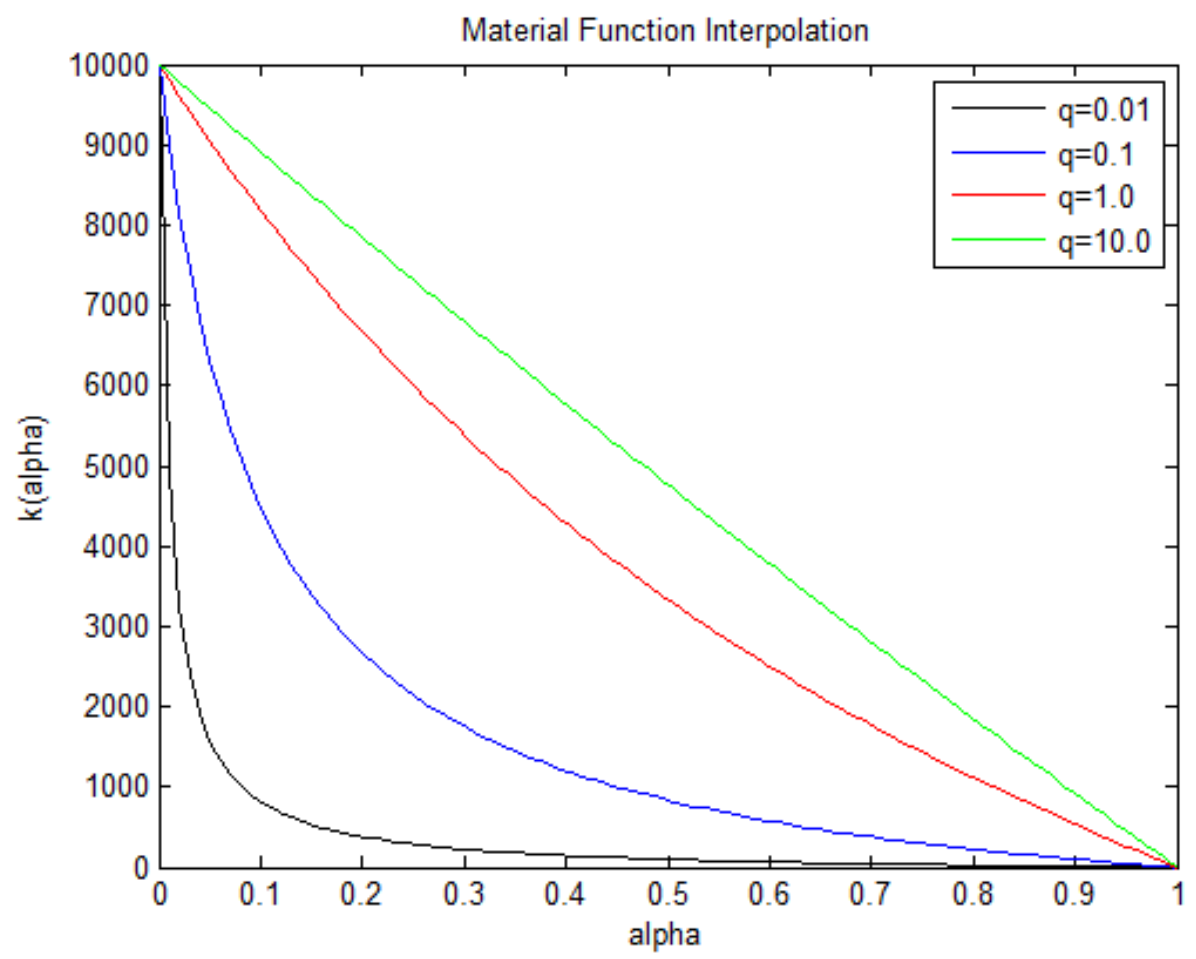

\subsubsection{Topology Optimization Problem}

The optimization problem in this work has the following formulation

$$
\text { Minimize : } c(\mathbf{z}(\alpha), \alpha)
$$

$\alpha$

subjected to : $\mathbf{g}(\mathbf{z}(\alpha), \alpha) \leq 0$

$$
\begin{aligned}
& \mathbf{r}(\mathbf{z}(\alpha), \alpha)=0 \\
& \alpha_{\min } \leq \alpha \leq \alpha_{\max }
\end{aligned}
$$


The function $c(\mathbf{z}(\alpha), \alpha)$ is a multi-objective that includes minimization of energy dissipation in the rotor, minimization of vorticity in the rotor and minimization power consumed in case of a pump or maximization of generated power in case of a turbine, respectively. Besides, some constraints are defined on Equation 3.2. A volume constraint is defined to restrict the amount of fluid regions on the domain, so a fraction of the domain volume $(f|\Omega|)$ is defined as an upper bound to create regions of fluid and leaving the remainder to be occupied by solid. Thus,

$$
\int_{\Omega} \alpha d \Omega \leq f|\Omega|
$$

where $f$ is the prescribed volume fraction, a constant between 0 and 1 .

\section{Energy Dissipation}

Energy loss minimization is very important for a great number of applications, specially applications with energy source restrictions, such as portable and small-sized applications, for example, cardiac pumps. Accordingly these pumps demand a better performance from the flow channels.

Minimizing the energy dissipation is one of the objective functions in this work, it represents the loss in fluid potential power and it is obtained through the principle of virtual work applied to the equilibrium equations. Also, minimizing the dissipated power in the fluid is equivalent to minimize the average pressure drop, under the assumption that velocities are uniform and normal to the domain boundary BORRVALL; PETERSSON 2003). Borvall and Petersson proposed a form for the energy dissipation that considers the effect of material model, given by:

$$
\boldsymbol{\Phi}=\int_{\Omega}\left[\frac{1}{2} \mu\left(\nabla \mathbf{u}+\nabla \mathbf{u}^{T}\right):\left(\nabla \mathbf{u}+\nabla \mathbf{u}^{T}\right)+\kappa(\alpha) \mathbf{u}^{2}\right] d \Omega
$$

where $\mathbf{u}$ is the relative velocity field on the rotating system.

\section{Vorticity}

Rotating systems have a high tendency to vortex formations that causes a swirl motion on the fluid generating a undesirable vortic current, implying on pressure loss and flow slip. Besides that, this reverse current can cause local cavitation (FRASER, 2001).

Vorticity represents the shear stress in the fluid and a high vorticity can damage particles flowing in the current, causing a loss of material integrity, due to fluid mixing. It can be hazardous to sensitive fluids such as blood.

Considering the inertial reference system, the rotor rotation contributes to vortex formation, in axial direction, through Coriolis parameter, i.e., contributes with $\boldsymbol{f}=2 \boldsymbol{\Omega}$. 
Thus, the absolute vorticity $\eta$ in the inertial reference is equal to the sum of relative vorticity $\epsilon$ with rotor vorticity $f$ :

$$
\eta=\epsilon+\boldsymbol{f}=\nabla \times \mathbf{u}+2 \boldsymbol{\Omega}
$$

In this work, only relative vorticity will be considered, and for a bi-dimensional case, the expression is given by (WHITE, 2001):

$$
\epsilon=\nabla \times \mathbf{u}=\left(\frac{\partial u_{2}}{\partial x_{1}}-\frac{\partial u_{1}}{\partial x_{2}}\right) e_{3}
$$

where $u_{1}$ and $u_{2}$ are relative velocity components on $x_{1}$ and $x_{2}$ direction, respectively.

A functional form for vorticity is expressed by (QUARTERONI; ROZZA, 2003; ABRAHAM; BEHR; HEINKENSCHLOSS, 2004):

$$
J(\mathbf{u})=\int_{\Omega}|\nabla \times \mathbf{u}|^{2} d \Omega=\int_{\Omega}\left(\frac{\partial u_{2}}{\partial x_{1}}-\frac{\partial u_{1}}{\partial x_{2}}\right)^{2} d \Omega
$$

\section{Power of Flow Machine (Pumps and Turbines)}

Flow machine power is a very important factor to optimize, because it influences the overall efficiency of energy generation, in case of turbines, or energy consumption, in case of pumps. So for turbines it is important to maximize the power, while for pumps it is necessary to minimize the power. This way, a direct correlation between power and performance can be established. The power related to a flow machine is directly proportional to the external torque (consumption or generated) and the angular velocity, represented by the expression:

$$
\dot{W}=\Omega \cdot \mathbf{T}_{e x t}
$$

Given that angular velocity is considered constant in this work, the objective function simplifies to torque only. Choosing a fixed control volume enclosing the rotor for analysis of torque reactions, and considering that surface forces do not contribute for torque, also that the body forces contribution can be neglected due to symmetry and that mass flow, in the inlet (suction) surface, is normal to the boundary, the total torque for steady state flow will be equal to the integral of the boundary outlet flow, i.e. (FOX; PRITCHARD; MCDONALD, 2008):

$$
\mathbf{T}_{e x t}=\int_{\Omega} \nabla \cdot\left[\rho\left(\mathbf{r} \times \mathbf{u}_{a}\right) \otimes \mathbf{u}_{a}\right] d \Omega=\int_{\Gamma_{e}} \rho\left(\mathbf{r} \times \mathbf{u}_{a}\right) \mathbf{u}_{a} \cdot \mathbf{n} d \Gamma
$$

where $\mathbf{u}_{a}$ is the absolute velocity $\left(\mathbf{u}_{a}=\mathbf{u}+\Omega \times \mathbf{r}\right)$. Given that $\Omega \times \mathbf{r}$ is tangent at the 
contour, $(\Omega \times \mathbf{r}) \cdot \mathbf{n}=0$ and the equation becomes:

$$
\mathbf{T}_{e x t}=\int_{\Gamma_{e}} \rho\left(\mathbf{r} \times \mathbf{u}_{a}\right) \mathbf{u} \cdot \mathbf{n} d \Gamma
$$

where $\mathbf{u}_{a}$ is the absolute velocity, $\Gamma_{e}$ is the boundary at flow exit and $\mathbf{n}$ is the outward unit normal vector of the domain. From Equation 2.3 (ROMERO; SILVA, 2014):

$$
\mathbf{T}_{e x t}=\int_{\Gamma_{e}} \rho(\mathbf{r} \times(\mathbf{u}+\mathbf{\Omega} \times \mathbf{r})) \mathbf{u} \cdot \mathbf{n} d \Gamma
$$

thus, being

$$
\begin{aligned}
\mathbf{T}_{r} & =\int_{\Gamma_{e}} \rho(\mathbf{r} \times \mathbf{u}) \mathbf{u} \cdot \mathbf{n} d \Gamma \\
\mathbf{T}_{t} & =\int_{\Gamma_{e}} \rho(\mathbf{r} \times \mathbf{\Omega} \times \mathbf{r}) \mathbf{u} \cdot \mathbf{n} d \Gamma \\
\mathbf{T}_{e x t} & =\mathbf{T}_{r}+\mathbf{T}_{t}=T_{r} \mathbf{e}_{3}+T_{t} \mathbf{e}_{3}=T_{e} \mathbf{e}_{3}
\end{aligned}
$$

where $T_{r} \mathbf{e}_{3}$ and $T_{t} \mathbf{e}_{3}$ are the $\mathrm{z}$ component of $\mathbf{T}_{r}$ and $\mathbf{T}_{t}$, respectively.

\section{Volume Restriction}

Besides the objective functions, a volume constraint is also defined as

$$
\sum_{i=1}^{n e} \alpha_{i} a_{i} \leq f V^{*}
$$

where ne is the total number of elements, $\alpha_{i}$ is the design variable, $a_{i}$ is the area of the element $i, 0<f \leq 1$ is a fraction coefficient and $V^{*}$ is the total volume to be considered.

\subsubsection{Multi-Objective Function}

The three objective equations previously defined are important for rotor design. Hence, they will be combined by defining a multi-objective function based on the weighting sum method :

$$
\Psi=\omega_{e} \Phi+\omega_{v} J \pm \omega_{t} T_{e}
$$

where $\Psi$ is the multi-objective function, $\Phi$ is the viscous energy dissipation term, $J$ is the vorticity and finally $T_{e}$ is the torque, representing the power functional. Some weighting coefficients are defined in order to determine different relevance between functionals, where $\omega_{e}, \omega_{v}$ and $\omega_{t}$ are associated with energy dissipation, vorticity and torque term, respectively. It is considered that $\omega_{e}+\omega_{v}+\omega_{t}=1.0$.

The term related to torque can assume two signals, the positive representing minimization 
of power in case of pumps and the negative corresponding to maximization of power for turbines.

The functionals of the multi-objective function (energy dissipation, vorticity and power) given in 3.16 have different orders of magnitude. Thus, in order to reduce these differences, two coefficients can be introduced (ZHU; ZHANG; FATIKOW, 2014):

$$
\Psi=\omega_{e} \Phi+\omega_{v} \beta_{k} J \pm \omega_{t} \gamma_{k} T_{e}
$$

where $\beta_{k}$ and $\gamma_{k}$ are weighting factors which change each iteration $\mathrm{k}$ of the optimization algorithm, and are defined as:

$$
\begin{gathered}
\beta_{k}=\frac{\Phi_{k-1}}{J_{k-1}}, \quad k \geq 1 \\
\gamma_{k}=\frac{\Phi_{k-1}}{T_{e, k-1}}, \quad k \geq 1
\end{gathered}
$$

where $\Phi_{k-1}, J_{k-1}$ and $T_{e, k-1}$ are the values of $\Phi, J$ and $T_{e}$ in the $(k-1)-t h$ iteration, respectively, and $\mathrm{k}=0$ is the functional values for the initial domain distribution.

It is important to notice that the energy dissipation functional is the main term in this multi-objective function, which gives the main format to the topology, while the other two terms essentially change the contour. Thus, in all weight combinations the energy dissipation is present, then, four cases can be evaluated:

$$
\begin{aligned}
& \Psi_{1}=\Phi \\
& \Psi_{2}=\omega_{e} \Phi+\omega_{v} \beta_{k} J \\
& \Psi_{3}=\omega_{e} \Phi+ \pm \omega_{t} \gamma_{k} T_{e} \\
& \Psi_{4}=\omega_{e} \Phi+\omega_{v} \beta_{k} J \pm \omega_{t} \gamma_{k} T_{e}
\end{aligned}
$$

\subsection{Discrete Forms of Energy dissipation, Vorticity and Power}

\subsubsection{Energy Dissipation Discrete Form}

As presented in Section 3.3.2, the energy dissipation is one of the functionals to be optimized. Its discrete form is given by (ROMERO; SILVA, 2014):

$$
\boldsymbol{\Phi}=\frac{1}{2} \mathbf{u}^{T}\left(\overline{\mathbf{K}}_{d}+\overline{\mathbf{K}}_{\kappa}\right) \mathbf{u}=\frac{1}{2} \mathbf{z}^{T} \mathbf{C z}
$$


where

$$
\begin{aligned}
\mathbf{C} & =\left[\begin{array}{cc}
\overline{\mathbf{K}}_{d}+\overline{\mathbf{K}}_{\kappa} & 0 \\
0 & 0
\end{array}\right] \\
\overline{\mathbf{K}}_{d} & =\left[\begin{array}{cc}
2 \mathbf{K}_{11}+\mathbf{K}_{22} & \mathbf{K}_{12} \\
\mathbf{K}_{21} & \mathbf{K}_{11}+2 \mathbf{K}_{22}
\end{array}\right] \\
\overline{\mathbf{K}}_{\kappa} & =\left[\begin{array}{cc}
\mathbf{K}_{\kappa} & 0 \\
0 & \mathbf{K}_{\kappa}
\end{array}\right],
\end{aligned}
$$

being $\mathbf{K}_{i j}$ and $\mathbf{K}_{\kappa}$ defined in Equation 2.13 .

\subsubsection{Vorticity Discrete Form}

The discrete form of vorticity implies in a numerical integration of Equation 3.7, giving ROMERO; SILVA, 2014):

$$
J(\mathbf{u})=\sum_{e=1}^{n e} \int_{\Omega^{e}}\left(\frac{\partial u_{2}}{\partial x_{1}}-\frac{\partial u_{1}}{\partial x_{2}}\right)^{2} d \Omega=\sum_{e=1}^{n e} \mathbf{U}^{T} \mathbf{M}_{\nu} \mathbf{U}
$$

where $n e$ is the total number of elements, $\mathbf{U}=\left[\mathbf{u}_{1} \mathbf{u}_{2}\right]^{T}$, and $\mathbf{u}_{1}, \mathbf{u}_{2}$ are vectors with nodal values of velocity in the $x_{1}$ and $x_{2}$ directions respectively. The local matrix $\mathbf{M}_{\nu}$ is defined by

$$
\mathbf{M}_{\nu}=\left[\begin{array}{ll}
\mathbf{M}_{\nu}^{11} & \mathbf{M}_{\nu}^{12} \\
\mathbf{M}_{\nu}^{21} & \mathbf{M}_{\nu}^{22}
\end{array}\right]
$$

where

$$
\begin{array}{rlrl}
\mathbf{M}_{\nu}^{11} & =\int_{\Omega^{e}}\left(\frac{\partial \boldsymbol{\Psi}}{\partial x_{2}} \frac{\partial \boldsymbol{\Psi}^{T}}{\partial x_{2}}\right) d \Omega ; & \mathbf{M}_{\nu}^{12}=-\int_{\Omega^{e}}\left(\frac{\partial \boldsymbol{\Psi}}{\partial x_{2}} \frac{\partial \boldsymbol{\Psi}^{T}}{\partial x_{1}}\right) d \Omega \\
\mathbf{M}_{\nu}^{21}=-\int_{\Omega^{e}}\left(\frac{\partial \boldsymbol{\Psi}}{\partial x_{1}} \frac{\partial \boldsymbol{\Psi}^{T}}{\partial x_{2}}\right) d \Omega ; & \mathbf{M}_{\nu}^{22}=\int_{\Omega^{e}}\left(\frac{\partial \boldsymbol{\Psi}}{\partial x_{1}} \frac{\partial \boldsymbol{\Psi}^{T}}{\partial x_{1}}\right) d \Omega
\end{array}
$$

where $\boldsymbol{\Psi}=\left[\phi_{1}, \ldots, \phi_{n}\right]^{T}$ and $n$ is the number of local nodes.

\subsubsection{Power Discrete Form}

As seen in Section 3.3.2, the power functional can be approximated by the torque applied, in case of a pump, or generated, in case of a turbine. Thus, following Equation 3.12, the torque discrete form is given by (ROMERO; SILVA, 2014):

$$
\mathbf{T}_{e x t}=\sum_{e=1}^{n b c}\left(T_{r} \mathbf{e}_{3}+T_{t} \mathbf{e}_{3}\right)
$$


where, $n b c$ is the number of elements in the outlet, and $T_{r}$ and $T_{t}$ are expressions for the local torque.

The normal vector, for any element on the boundary, considering that $\gamma$ is a local coordinate over the boundary, is given by:

$$
\mathbf{n}=\frac{\nabla \gamma}{|\nabla \gamma|}
$$

and

$$
\frac{d \Gamma}{d \gamma}=\left[\left(\frac{d x_{1}}{d \gamma}\right)^{2}+\left(\frac{d x_{2}}{d \gamma}\right)^{2}\right]^{\frac{1}{2}}
$$

Thus,

$$
\mathbf{u} \cdot \mathbf{n}=\left(\frac{d \Gamma}{d \gamma}\right)^{-1}\left(u_{1} \frac{d x_{2}}{d \gamma}-u_{2} \frac{d x_{1}}{d \gamma}\right)
$$

and

$$
\mathbf{r} \times \mathbf{u}=\left(x_{1} u_{2}-x_{2} u_{1}\right) \mathbf{e}_{3}
$$

Which gives

$$
T_{r}=\int_{\Gamma_{i}} \rho\left(\frac{d \Gamma}{d \gamma}\right)^{-1}\left(u_{1} \frac{d x_{2}}{d \gamma}-u_{2} \frac{d x_{1}}{d \gamma}\right)\left(x_{1} u_{2}-x_{2} u_{1}\right) d \Gamma
$$

By using local coordinates the integral becomes

$$
\begin{aligned}
T_{r} & =\int_{-1}^{1} \rho\left(\frac{d \Gamma}{d \gamma}\right)^{-1}\left(u_{1} \frac{d x_{2}}{d \gamma}-u_{2} \frac{d x_{1}}{d \gamma}\right)\left(x_{1} u_{2}-x_{2} u_{1}\right) \frac{d \Gamma}{d \gamma} d \gamma \\
& =\int_{-1}^{1} \rho\left(u_{1} \frac{d x_{2}}{d \gamma}-u_{2} \frac{d x_{1}}{d \gamma}\right)\left(x_{1} u_{2}-x_{2} u_{1}\right) d \gamma \\
& =\int_{-1}^{1} \rho\left[-u_{1} x_{2} \frac{d x_{2}}{d \gamma} u_{1}+u_{1} x_{2} \frac{d x_{1}}{d \gamma} u_{2}+u_{2} x_{1} \frac{d x_{2}}{d \gamma} u_{1}-u_{2} x_{1} \frac{d x_{1}}{d \gamma} u_{2}\right] d \gamma
\end{aligned}
$$

Finally, the torque $T_{r}$ per element is given by

$$
T_{r}=\mathbf{U}^{T} \mathbf{M}_{r} \mathbf{U}
$$

where, $\mathbf{U}=\left[\mathbf{u}_{1} \mathbf{u}_{2}\right]^{T}$, and $\mathbf{u}_{1}, \mathbf{u}_{2}$ are vectors with nodal values of velocity in the $x_{1}$ and $x_{2}$ directions respectively. The local matrix $\mathbf{M}_{r}$, obtained from Equation 3.37, is defined as

$$
\mathbf{M}_{r}=\left[\begin{array}{ll}
M_{r i j}^{11} & M_{r i j}^{12} \\
M_{r i j}^{21} & M_{r i j}^{22}
\end{array}\right]
$$


where

$$
\begin{array}{rlrl}
M_{r i j}^{11}=-\int_{-1}^{1} \rho\left(\phi_{i} x_{2} \frac{d x_{2}}{d \gamma} \phi_{j}\right) d \gamma ; & M_{r i j}^{12}=\int_{-1}^{1} \rho\left(\phi_{i} x_{2} \frac{d x_{1}}{d \gamma} \phi_{j}\right) d \gamma \\
M_{r i j}^{21}=\int_{-1}^{1} \rho\left(\phi_{i} x_{1} \frac{d x_{2}}{d \gamma} \phi_{j}\right) d \gamma ; & M_{r i j}^{22}=-\int_{-1}^{1} \rho\left(\phi_{i} x_{1} \frac{d x_{1}}{d \gamma} \phi_{j}\right) d \gamma \\
i, j=1, \ldots, n b
\end{array}
$$

where $n b$ is the number of nodes in the local contour elements.

The torque integral $T_{t}$ per element can be expressed by

$$
T_{t}=-\int_{-1}^{1} \rho \Omega_{0}(\mathbf{r} \cdot \mathbf{r})\left(u_{1} \frac{d x_{2}}{d \gamma}-u_{2} \frac{d x_{1}}{d \gamma}\right) d \gamma
$$

which in the discrete form is given by

$$
T_{t}=\mathbf{V}^{T} \mathbf{U}
$$

where $\mathbf{V}=\left[\mathbf{V}^{1} \mathbf{V}^{2}\right]^{T}$, and

$$
\begin{array}{r}
V_{i}^{1}=-\int \rho \Omega_{0}\left(\left(x_{1}^{2}+x_{2}^{2}\right) \frac{d x_{2}}{d \gamma} \phi_{i}\right) d \gamma ; \quad V_{i}^{2}=\int \rho \Omega_{0}\left(\left(x_{1}^{2}+x_{2}^{2}\right) \frac{d x_{1}}{d \gamma} \phi_{i}\right) d \gamma \\
i=1, \ldots, n b
\end{array}
$$




\section{SENSITIVITY OF TOPOLOGY OPTIMIZATION PROBLEM}

This chapter describes the sensitivity calculation for all the functionals defined in Chapter 2 with respect to the design variable. The sensitivities are used to perform the optimization, as shown in Chapter 5.

\subsection{Adjoint Method}

The gradients with respect to the design variables are required for the optimization algorithm to define the topology. In this work the adjoint approach is used. The adjoint method creates a problem given by the derivation of the governing equation in weak form, and allows obtaining the functional gradient by using the derivatives with respect to the output variables.

Considering a system of PDE, $F(u, m)=0$, and a functional of interest, $J(u(m), m)$, where $u$ is the output of PDE solution and $m$ is the design variable (input parameter), the derivative of $J$ with respect to $m$ can be calculated by the chain rule (FARRELL et al. 2013):

$$
\frac{d J}{d m}=\frac{\partial J}{\partial u} \frac{d u}{d m}+\frac{\partial J}{\partial m}
$$

where the derivative of $u$ with respect to $m$ may be obtained from the original system of PDE:

$$
\begin{array}{r}
\frac{\partial F}{\partial u} \frac{d u}{d m}+\frac{\partial F}{\partial m}=0 \\
\frac{d u}{d m}=-\left(\frac{\partial F}{\partial u}\right)^{-1} \frac{\partial F}{\partial m}
\end{array}
$$

Therefore, the derivative of the functional $J$. with respect to $m$, is given by:

$$
\frac{d J}{d m}=-\frac{\partial J}{\partial u}\left(\frac{\partial F}{\partial u}\right)^{-1} \frac{\partial F}{\partial m}+\frac{\partial J}{\partial m}
$$

Regrouping the derivative with respect to $u$, we define the adjoint vector $\lambda^{*}$ :

$$
\lambda^{*}=\frac{\partial J}{\partial u}\left(\frac{\partial F}{\partial u}\right)^{-1}
$$

in which $*$ represents the complex conjugate of the transpose and, thus, the following 
adjoint problem is stated:

$$
\frac{\partial F^{*}}{\partial u} \lambda=\frac{\partial J^{*}}{\partial u}
$$

Accordingly, the functional sensitivity is:

$$
\frac{d J}{d m}=-\lambda^{*} \frac{\partial F}{\partial m}+\frac{\partial J}{\partial m}
$$

Hence, the adjoint solution vector, $\lambda$, can be used to compute the gradients of the functional $J$ with respect to any set of parameters $m$.

\subsection{Sensitivity Analysis by the Adjoint Method}

The sensitivity analysis consists of gradient evaluation of the objective functions in order to provide information about the function and the constraints to guide the optimization. In this work, a multi-objective function is defined (Equation 3.17) and its gradient is given by:

$$
\frac{d \Psi}{d \alpha_{i}}=w_{e} \frac{d \Phi}{d \alpha_{i}}+w_{v} \beta^{k} \frac{d J}{d \alpha_{i}}+w_{v} \frac{d \beta^{k}}{d \alpha_{i}} J+w_{t} \gamma^{k} \frac{d T_{e}}{d \alpha_{i}}+w_{t} \frac{d \gamma^{k}}{d \alpha_{i}} T_{e}
$$

The terms $\frac{d \beta^{k}}{d \alpha_{i}}$ and $\frac{d \gamma^{k}}{d \alpha_{i}}$ are the sensitivities of $\beta^{k}$ and $\gamma^{k}$ with respect to $\alpha$, respectively, that arises due to the derivative chain rule. Considering that $\beta^{k}$ and $\gamma^{k}$ are restricted to be constant at each individual optimization iteration, and only $\Phi, J$ and $T_{e}$ are treated to be variables, the sensitivities for $\beta^{k}$ and $\gamma^{k}$ can be obtained by (ZHU; ZHANG; FATIKOW, 2014):

$$
\begin{aligned}
& \frac{\partial \beta^{k}}{\partial \alpha_{i}}=0 \\
& \frac{\partial \gamma^{k}}{\partial \alpha_{i}}=0
\end{aligned}
$$

The calculation of sensitivities $\frac{d \Phi}{d \alpha_{i}}, \frac{d J}{d \alpha_{i}}$ and $\frac{d T_{e}}{d \alpha_{i}}$ is described in the following sections.

\subsubsection{Energy Dissipation Sensitivity analysis}

Using the adjoint method described in Section 4.1, it is possible to determine analytically the gradient of energy dissipation functional with respect to the design variable $\alpha_{i}$. In this case, the matrix $\mathbf{C}$ is symmetric, and therefore $\mathbf{C}^{T}=\mathbf{C}$. Thus, by differentiating the Equation 3.24 (ROMERO; SILVA, 2014):

$$
\frac{\partial \Phi}{\partial \alpha_{i}}=\frac{1}{2}\left\{\left(\frac{\partial \mathbf{z}}{\partial \alpha_{i}}\right)^{T} \mathbf{C} \mathbf{z}+\mathbf{z}^{T} \frac{\partial \mathbf{C}}{\partial \alpha_{i}} \mathbf{z}+\mathbf{z}^{T} \mathbf{C} \frac{\partial \mathbf{z}}{\partial \alpha_{i}}\right\}=\frac{1}{2} \mathbf{z}^{T} \frac{\partial \mathbf{C}}{\partial \alpha_{i}} \mathbf{z}+\mathbf{z}^{T} \mathbf{C} \frac{\partial \mathbf{z}}{\partial \alpha_{i}}
$$


From the discrete FEM system equations 2.12 , the term $\frac{\partial \mathbf{z}}{\partial \alpha_{i}}$ can be obtained by differentiating its residual. Hence, given the residual:

$$
\mathbf{R}=\mathbf{K}_{G} \mathbf{z}-\mathbf{F}
$$

where,

$$
\mathbf{K}_{G}=\left[\begin{array}{cc}
\mathbf{C}(\mathbf{u})+\mathbf{K} & -\mathbf{Q} \\
-\mathbf{Q}^{T} & 0
\end{array}\right] ; \quad \mathbf{z}=\left[\begin{array}{ll}
\mathbf{u} & \mathbf{p}
\end{array}\right]^{T} ; \quad \mathbf{F}=\left[\begin{array}{ll}
\mathbf{f} & \mathbf{0}
\end{array}\right]^{T}
$$

and $\mathbf{u}$ and $\mathbf{p}$ are vectors with the nodal values of velocity and pressure of finite element method approximate solution, respectively.

The differentiation with respect to $\alpha_{i}$ becomes:

$$
\frac{d \mathbf{R}}{\alpha_{i}}=\frac{\partial \mathbf{R}}{\partial \mathbf{z}} \frac{d \mathbf{z}}{\alpha_{i}}+\frac{\partial \mathbf{R}}{\partial \alpha_{i}}=0
$$

where $\frac{\partial \mathbf{R}}{\partial \mathbf{z}}=\mathbf{J}$, is the Jacobian matrix resulting from the application of Newton's method to the solution of the nonlinear system.

So, substituting $\mathbf{J}$ in Equation 4.13 , we obtain:

$$
\mathbf{J} \frac{d \mathbf{z}}{\alpha_{i}}=-\frac{\partial \mathbf{R}}{\partial \alpha_{i}}=-\frac{\partial \mathbf{K}_{G}}{\partial \alpha_{i}} \mathbf{z}+\frac{\partial \mathbf{F}}{\partial \alpha_{i}}=\mathbf{r}_{i}
$$

Therefore, from Equation 4.14 .

$$
\frac{d \mathbf{z}}{\alpha_{i}}=\mathbf{J}^{-1} \mathbf{r}_{i}
$$

Replacing this expression in the second term of Equation 4.10 .

$$
\mathbf{z}^{T} \mathbf{C} \frac{\partial \mathbf{z}}{\partial \alpha_{i}}=\mathbf{z}^{T} \mathbf{C} \mathbf{J}^{-1} \mathbf{r}_{i}=\mathbf{S}^{T} \mathbf{r}_{i}
$$

where,

$$
\mathbf{S}^{T}=\mathbf{z}^{T} \mathbf{C} \mathbf{J}^{-1}
$$

Rearranging this expression, the following linear system is obtained:

$$
\mathbf{J}^{T} \mathbf{S}=\mathbf{C} \mathbf{z}
$$

If we solve the linear system 4.18 the vector $\mathbf{S}$ is obtained, then, backtracking to Equation 4.16 the second term Equation 4.10 is calculated and consequently the energy dissipation sensitivity can be obtained. The system from Equation 4.18 is independent of 
the derivative with respect to the design variable, so it is solved once per iteration of the optimization process, using the velocity (U) field from the previous topology.

It is important to notice that the residual $\mathbf{R}$ is assumed to be zero, given that the velocities result from the Newton converged process. Also, the vector $\mathbf{r}_{i}$, from Equations 4.16 and 4.14 , is calculated for each element $i$ in the domain.

\subsubsection{Vorticity Sensitivity Analysis}

Using the same method from the previous section, the gradient of vorticity functional with respect to the design variable $\alpha_{i}$ can be calculated, and is expressed by differentiating Equation 3.28 with respect to the design variable (ROMERO; SILVA, 2014):

$$
\frac{d J}{d \alpha_{i}}=\frac{d \mathbf{U}^{T}}{d \alpha_{i}} \mathbf{M}_{\nu} \mathbf{U}+\mathbf{U}^{T} \frac{\partial \mathbf{M}_{\nu}}{\partial \alpha_{i}} \mathbf{U}+\mathbf{U}^{T} \mathbf{M}_{\nu} \frac{d \mathbf{U}}{d \alpha_{i}}
$$

given that $\mathbf{M}_{\nu}$ is not a direct function of $\alpha_{i}, \frac{\partial \mathbf{M}_{\nu}}{\partial \alpha_{i}}=0$, thus

$$
\frac{d J}{d \alpha_{i}}=\mathbf{U}^{T}\left(\mathbf{M}_{\nu}+\mathbf{M}_{\nu}^{T}\right) \frac{d \mathbf{U}}{d \alpha_{i}}
$$

The term $\frac{d \mathbf{U}}{d \alpha_{i}}$ can be obtained from the FEM equilibrium equation system, using an auxiliary matrix to isolate the velocities from the vector $\mathbf{z}$, i.e.:

$$
\mathbf{U}=\mathbf{L}^{T} \mathbf{z} \Longrightarrow \frac{d \mathbf{U}}{\alpha_{i}}=\mathbf{L}^{T} \frac{d \mathbf{z}}{\alpha_{i}}
$$

where

$$
\mathbf{L}^{T}=\left[\begin{array}{ll}
\mathbf{I}_{n \times n} & \mathbf{0}_{n \times m}
\end{array}\right]
$$

being the $\mathbf{L}$ matrix composed by the identity matrix $\mathbf{I}$, of dimensions $(n \times n)$, where $n$ is the number of degrees of freedom of velocity, and an array of dimension $(n \times m)$ consisting only of zeros, where $m$ is the number of degrees of freedom of pressure.

Hence, from equations 4.15 and 4.21 .

$$
\frac{d \mathbf{U}}{d \alpha_{i}}=\mathbf{L}^{T} \frac{d \mathbf{z}}{\alpha_{i}} \Longrightarrow \frac{d \mathbf{U}}{d \alpha_{i}}=\mathbf{L}^{T} \mathbf{J}^{-1} \mathbf{r}_{i}
$$

going back to Equation 4.20 and replacing the Equation 4.23 on it:

$$
\frac{d J}{d \alpha_{i}}=\mathbf{U}^{T}\left(\mathbf{M}_{\nu}+\mathbf{M}_{\nu}^{T}\right) \mathbf{L}^{T} \mathbf{J}^{-1} \mathbf{r}_{i}=\mathbf{S}_{v}^{T} \mathbf{r}_{i}
$$


where,

$$
\mathbf{S}_{v}^{T}=\mathbf{U}^{T}\left(\mathbf{M}_{\nu}+\mathbf{M}_{\nu}^{T}\right) \mathbf{L}^{T} \mathbf{J}^{-1}
$$

Rearranging this expression, the following linear system is obtained:

$$
\mathbf{J}^{T} \mathbf{S}_{v}=\mathbf{L}\left(\mathbf{M}_{\nu}+\mathbf{M}_{\nu}^{T}\right) \mathbf{U}
$$

Similarly to the energy sensitivity system deduction (Equation 4.18), the $\mathbf{S}_{v}$ vector is obtained by solving the linear system. This system is also independent of the derivative with respect to the design variable, so it is solved once per iteration of the optimization process. It is also dependent on the previous velocity field $\mathbf{U}$ and the residual $\mathbf{R}$ is assumed to be zero. And it is important to remark that the vector $\mathbf{r}_{i}$, from equations 4.24 , given by 4.14 , is calculated for each element $i$ in the domain.

\subsubsection{Power Sensitivity Analysis}

The gradient of power functional with respect to the design variable can be obtained by solving the adjoint problem. Thus, the total power can be expressed by equations 3.40 and 3.44 (ROMERO; SILVA, 2014):

$$
T_{e}=\mathbf{U}^{T} \mathbf{M}_{r} \mathbf{U}+\mathbf{V}^{T} \mathbf{U}
$$

which can be differentiated with respect to the design variable $\alpha_{i}$, giving:

$$
\frac{d T_{e}}{\alpha_{i}}=\frac{d \mathbf{U}^{T}}{\alpha_{i}} \mathbf{M}_{r} \mathbf{U}+\mathbf{U}^{T} \frac{\partial \mathbf{M}_{r}}{\partial \alpha_{i}} \mathbf{U}+\mathbf{U}^{T} \mathbf{M}_{r} \frac{d \mathbf{U}}{\alpha_{i}}+\mathbf{V}^{T} \frac{d \mathbf{U}}{\alpha_{i}}
$$

as $\frac{\partial \mathbf{M}_{r}}{\partial \alpha_{i}}=0$, because $\mathbf{M}_{r}$ is not a direct function of $\alpha_{i}$. Also, $\mathbf{M}_{r}$ is not symmetrical, so Equation 4.28 becomes:

$$
\frac{d T_{e}}{\alpha_{i}}=\mathbf{U}^{T}\left(\mathbf{M}_{r}+\mathbf{M}_{r}^{T}\right) \frac{d \mathbf{U}}{d \alpha_{i}}+\mathbf{V}^{T} \frac{d \mathbf{U}}{d \alpha_{i}}
$$

Using the definition of $\mathbf{L}$ (Eq. 4.22 ) and the Equation 4.23 .

$$
\frac{d \mathbf{U}}{d \alpha_{i}}=\mathbf{L}^{T} \mathbf{J}^{-1} \mathbf{r}_{i}
$$

Equation 4.29 gives:

$$
\frac{d T_{e}}{\alpha_{i}}=\left[\mathbf{U}^{T}\left(\mathbf{M}_{r}+\mathbf{M}_{r}^{T}\right)+\mathbf{V}^{T}\right] \mathbf{L}^{T} \mathbf{J}^{-1} \mathbf{r}_{i}=\mathbf{S}_{t}^{T} \mathbf{r}_{i}
$$


where

$$
\mathbf{S}_{t}^{T}=\left[\mathbf{U}^{T}\left(\mathbf{M}_{r}+\mathbf{M}_{r}^{T}\right)+\mathbf{V}^{T}\right] \mathbf{L}^{T} \mathbf{J}^{-1}
$$

Rearranging the previous expression, the following linear system is obtained:

$$
\mathbf{J}^{T} \mathbf{S}_{t}=\mathbf{L}\left[\mathbf{U}^{T}\left(\mathbf{M}_{r}+\mathbf{M}_{r}^{T}\right)+\mathbf{V}^{T}\right]
$$

Thus, by solving the above system the vector $\mathbf{S}_{t}$ is obtained. This system is independent of the derivative with respect to the design variable, so it is solved once per iteration of the optimization process. Also, this system is dependent on the velocity field $\mathbf{U}$ resulting from the previous domain configuration. The residual is assumed to be zero, so the velocity field used is the one from the converged Newton process.

In Equation 4.31, the vector $\mathbf{r}_{i}$ given by Equation 4.14, is calculated for each element $i$ in the domain.

\subsubsection{Multi-Objective Function Sensitivity Analysis}

Considering that the gradient of the multi-objective function, which is given by Equation 4.7, depends on the sensitivities of the energy dissipation, vorticity and power, and also remembering that each one of these terms depends on the respective adjoint problem, the final gradient can be calculated by solving each separate problem and then replacing the results on Equation 4.7. However, the three adjoint problems can be combined and only one final adjoint equation associated with the multi-objective function needs to be solved (ROMERO; SILVA, 2014).

Thus, by substituting Equation 4.16 in Equation 4.10, the energy dissipation sensitivity is:

$$
\frac{\partial \Phi}{\partial \alpha_{i}}=\frac{1}{2} \mathbf{z}^{T} \frac{\partial \mathbf{C}}{\partial \alpha_{i}} \mathbf{z}+\mathbf{z}^{T} \mathbf{C} \mathbf{J}^{-1} \mathbf{r}_{i}
$$

The vorticity sensitivity contribution (Eq. 4.24 ) is:

$$
\frac{d J}{d \alpha_{i}}=\mathbf{U}^{T}\left(\mathbf{M}_{\nu}+\mathbf{M}_{\nu}^{T}\right) \mathbf{L}^{T} \mathbf{J}^{-1} \mathbf{r}_{i}
$$

The power sensitivity contribution (Eq. 4.31) is:

$$
\frac{d T_{e}}{\alpha_{i}}=\left[\mathbf{U}^{T}\left(\mathbf{M}_{r}+\mathbf{M}_{r}^{T}\right)+\mathbf{V}^{T}\right] \mathbf{L}^{T} \mathbf{J}^{-1} \mathbf{r}_{i}
$$


Thus, substituting the above equations on Equation 4.7, it is obtained:

$$
\begin{aligned}
\frac{d \Psi}{\alpha_{i}}= & w_{e}\left[\frac{1}{2} \mathbf{z}^{T} \frac{\partial \mathbf{C}}{\partial \alpha_{i}} \mathbf{z}+\mathbf{z}^{T} \mathbf{C J}^{-1} \mathbf{r}_{i}\right]+w_{v} \beta^{k} \mathbf{U}^{T}\left(\mathbf{M}_{\nu}+\mathbf{M}_{\nu}^{T}\right) \mathbf{L}^{T} \mathbf{J}^{-1} \mathbf{r}_{i} \\
& +w_{t} \gamma^{k}\left[\mathbf{U}^{T}\left(\mathbf{M}_{r}+\mathbf{M}_{r}^{T}\right)+\mathbf{V}^{T}\right] \mathbf{L}^{T} \mathbf{J}^{-1} \mathbf{r}_{i}
\end{aligned}
$$

Grouping the terms:

$$
\begin{aligned}
\frac{d \Psi}{\alpha_{i}} & =\frac{1}{2} w_{e} \mathbf{z}^{T} \frac{\partial \mathbf{C}}{\partial \alpha_{i}} \mathbf{z} \\
& +\left[w_{e} \mathbf{z}^{T} \mathbf{C}+w_{v} \beta^{k} \mathbf{U}^{T}\left(\mathbf{M}_{\nu}+\mathbf{M}_{\nu}^{T}\right) \mathbf{L}^{T}+w_{t} \gamma^{k}\left(\mathbf{U}^{T}\left(\mathbf{M}_{r}+\mathbf{M}_{r}^{T}\right)+\mathbf{V}^{T}\right) \mathbf{L}^{T}\right] \mathbf{J}^{-1} \mathbf{r}_{i}
\end{aligned}
$$

Finally,

$$
\frac{d \Psi}{\alpha_{i}}=\frac{1}{2} w_{e} \mathbf{z}^{T} \frac{\partial \mathbf{C}}{\partial \alpha_{i}} \mathbf{z}+\mathbf{S}_{m}^{T} \mathbf{r}_{i}
$$

where $\mathbf{S}_{m}$ is obtained from the solution of the system:

$$
\mathbf{J}^{T} \mathbf{S}_{m}=\left[w_{e} \mathbf{C z}+w_{v} \beta^{k} \mathbf{L}\left(\mathbf{M}_{\nu} \mathbf{M}_{\nu}^{T}\right) \mathbf{U}+w_{t} \gamma^{k} \mathbf{L}\left[\left(\mathbf{M}_{\nu} \mathbf{M}_{\nu}^{T}\right) \mathbf{U}+\mathbf{V}\right]\right]
$$

In Equation 4.39, the vector $\mathbf{r}_{i}$, given by Equation 4.14, is calculated for each element $i$ in the domain, and $\mathbf{J}$ is the Jacobian matrix resulting from the application of Newton's method to the solution of the nonlinear system. 


\section{NUMERICAL IMPLEMENTATION}

\subsection{Optimization Algorithm}

The optimization algorithm can be a stochastic algorithm, which generates and uses random variables, such as genetic algorithms; or gradient based, where the gradient information with respect to the desired functional is evaluated and gives the direction to update the design variables values. In this work, a gradient based algorithm will be used.

The topology optimization procedure is described in Section 1.3, it consists essentially of FEM resolution, gradient evaluation and optimization algorithm. The procedure is iterative and consists of the following steps

(a) Solve Navier-Stokes equations with given initial value for design variables;

(b) Solve adjoint equations based on velocity and pressure fields of (a);

(c) Calculate the sensitivities of objective functions and constraints;

(d) Update the design variable value with an optimization algorithm;

(e) Verify the stopping criteria.

The stopping criteria usually is defined as a norm between two consecutive iterations, as

$$
\left\|\alpha^{k}-\alpha^{k-1}\right\|_{\infty} \ll 1 \times 10^{-3}
$$

where $\alpha$ is the design variable distribution and $k$ is the iteration number.

\subsection{Software Environment}

By using the equations presented in the previous sections it is possible to implement a software to solve the partial differential equations (PDE). There are a number of software capable of solving the PDEs by the Finite Element Method (FEM), some require a manual implementation, such as the commercial environment $M A T L A B \AA$, others have modules that receive the weak problem formulation and assemble the FEM problem, such as the commercial software COMSOL and the Open Source software FEniCS. In this work, the FEniCS environment and some external libraries, such as the libadjoint and the pyIPOPT, will be used. A more detailed explanation of these libraries is presented in the following sections. 


\subsubsection{Solving Differential Equations - The FEniCS system}

The FEniCS system (LOGG; WELLS; BOOK, 2012) is a collection of software components for automating the solution of PDEs by the finite element method. This involves translation of a mathematical syntax to a language that can be interpreted by the computer. So, aiming to use the weak formulation as a software input, it is necessary to use a software capable of interpreting this high-level language and transforming it into a numerical routine. This interpretation software is usually referred to as Form Compiler $(\mathrm{FC})$.

The FEniCS environment has its own form compiler, the FEniCS Form Compiler (FFC), that receives a discrete form of the weak variational equation given in Unified Form Language (UFL) (ALNÆS et al., 2014), similar to the mathematical formulation, and generates a $\mathrm{C}++$ code of the finite element assembly in the format of the Unified Form-Assembly Code (UFC) (ALNAES et al., 2009). This assembly is an optimised low-level code that evaluates the local element tensors.

The local tensors are used by DOLFIN (LOGG; WELLS, 2010), a library that handles the communication between all FEniCS modules. This library also provides various data structures to interface meshes, function spaces, functions and solvers. These tensors are coded in a format of SciPy, a Python-based Open Source software that has modules to handle scientific computing. This enables the use of many linear algebra packages, such as PETSc, Epetra and uBLAS, which permits to select among many linear solvers and pre-conditioners.

\subsubsection{Solvers and Preconditioners}

The FEM equations, in general, large sparse systems of linear equations, are solved by iterative methods. Many methods exist for solving these type of problems, and it is necessary to find one that is effective and reliable. Unfortunately a method that works well for one problem type might not work as well for another, sometimes it does not even converge.

The Navier-Stokes in the rotating reference system is a problem that hardly converges when the basic Newton method is used. Thus, more complex solvers are needed to guarantee the solution convergence. The FEniCS system offers pre-installed support to methods such as Generalized minimal residual (Gmres) and Biconjugate gradient stabilized (Bicstab), that are usually used on Navier-Stokes problems. However, some external solvers can be used, and in this work the MUltifrontal Massively Parallel Sparse direct Solver (MUMPS) (AMESTOY et al., 2001) has been chosen because it offers features such as input of the matrix in assembled format (distributed or centralized) or elemental format, error analysis, and parallel analysis. 


\subsubsection{Solution of the Adjoint Problem}

There are some methods to derive the adjoint model from the PDE. The first and more intuitive is to do the same steps of the manual manipulation of the forward equations. Some software try to mimic this by automating this by-hand manipulation, such as the $S A G E$ (STEIN; JOYNER, 2005). A second method is to derive at a source code level, treating the forward model as a sequence of elementary instructions (FARRELL et al., 2013). A third method consists of doing the derivation of the forward method at a middle-level approach, that can be automated without depending on low-level implementation details. These three approaches are presented on Figure 5.1, summarising the possible ways to derive the adjoint model starting from the forward equations, under the FEniCS environment.

Figure 5.1 - Methods for obtaining the adjoint code.

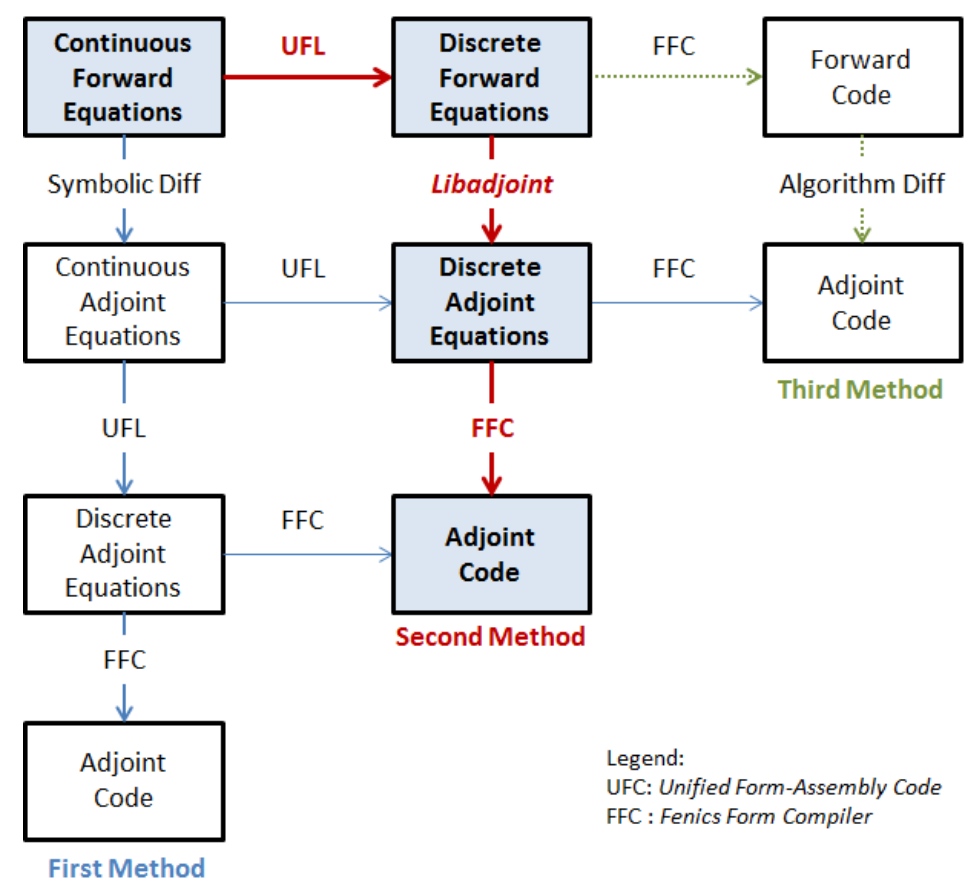

Adapted from Farrell et al. (2013)

The second method relies on the library libadjoint, that enables the automatic derivation and solution of tangent linear and adjoint models. The purpose of libadjoint is to facilitate the development of these models based on the fundamental abstraction of considering the forward model as a sequence of equation solutions. Based on this abstraction, the library builds a symbolic description of the forward model, from which it can automatically derive the symbolic representation of the associated tangent linear and adjoint systems (FUNKE; FARRELL, 2013).

In this work the path in bold in Figure 5.1 (Second Method) is used. Thus, the forward equations are discretized by using the UFL package, then the discrete adjoint equations are derived by using the libadjoint library and for last the adjoint code is assembled through the FFC package. Also, in order to interface DOLFIN with libadjoint there is the 
dolfin-adjoint software, that gets the adjoint model derived by the libadjoint and translates it to the same high-level language of the forward model, i.e., it does the connection between UFL and FFC.

\subsubsection{Solution of the Optimization Problem}

In this work, the topology optimization problem is implemented by using the framework formed by the FEniCS system, to solve the PDEs, the libadjoint library, to compute the adjoint model, and an optimizer, to update the domain. These software (FEniCS and libadjoint) are all coded in Python language, so, to simplify the implementation, the optimizer also has to be coded in this language. A series of optimizers offer compatibility with Python and FEniCS, such as the pyOpt package, that has some well-known optimizers as the MMA (SVANBERG, 1987) and the ALGENCAN. However, in this work another package is used, the IPOpt (WÄCHTER, 2002; WÄCHTER; BIEGLER, 2006, WÄCHTER, 2009), which implements the Internal Point Optimization algorithm.

The IPOpt software implements a primal-dual barrier method that solves a sequence of barrier problems. The complete algorithm can be found in (WÄCHTER, 2002), however, the approach for the barrier method is briefly described as follows. The generic minimization problem is given by Eq. 5.2 .

$$
\begin{aligned}
\text { Minimize : } & f(x) \\
x \in \mathbb{R}^{n} & \\
\text { subjected to : } & c(x)=0 \\
& x^{(i)} \geq 0 \quad \text { for } i \in I
\end{aligned}
$$

The barrier method replaces the bound constraints by a logarithmic barrier term which is added to the objective function giving:

$$
\begin{aligned}
\text { Minimize }: & \phi_{\mu}(x):=f(x)-\mu \sum_{i \in I} \ln \left(x^{(i)}\right) \\
x \in \mathbb{R}^{n} & \\
\text { subjected to : } & c(x)=0
\end{aligned}
$$

where $\mu>0$ is the barrier parameter, $\phi_{\mu}(x)$ is the barrier function and $-\mu \sum_{i \in I} \ln \left(x^{(i)}\right)$ is the barrier parameter.

The degree of influence of the barrier term is determined by the size of $\mu$, and the barrier problem solution eventually converges to the solution of the original problem as $\mu \rightarrow 0$. Thus, an initial value for the barrier parameter is defined and the barrier problem is solved, then the barrier parameter is updated and the the loop restarts. The loop is terminated when a tolerance for $\mu_{l+1}-\mu_{l}$ is reached. 
The solution of the barrier problem is done using two loops ( $l$ and $k$ ), each loop $(l)$ is done by an inner loop $(k)$ that computes the primal-dual search directions $\left(d_{k}, d_{k}^{v}\right)$ using a Newton's method, then define the step lengths $\left(\alpha_{k}, \alpha_{k}^{v} \in(0,1]\right)$ to obtain the next iterate $x_{k+1}:=x_{k}+\alpha_{k} d_{k}$ for the primal problem and $v_{k+1}:=v_{k}+\alpha_{k}^{v} d_{k}^{v}$ for the dual problem. The inner loop stops when $x_{k}$ is a local solution (or at least a stationary point) of the barrier problem, i.e., if it satisfies the KKT conditions (Karush-Kuhn-Tucker conditions).

Figure 5.2 - Topology optimization implementation flow chart.

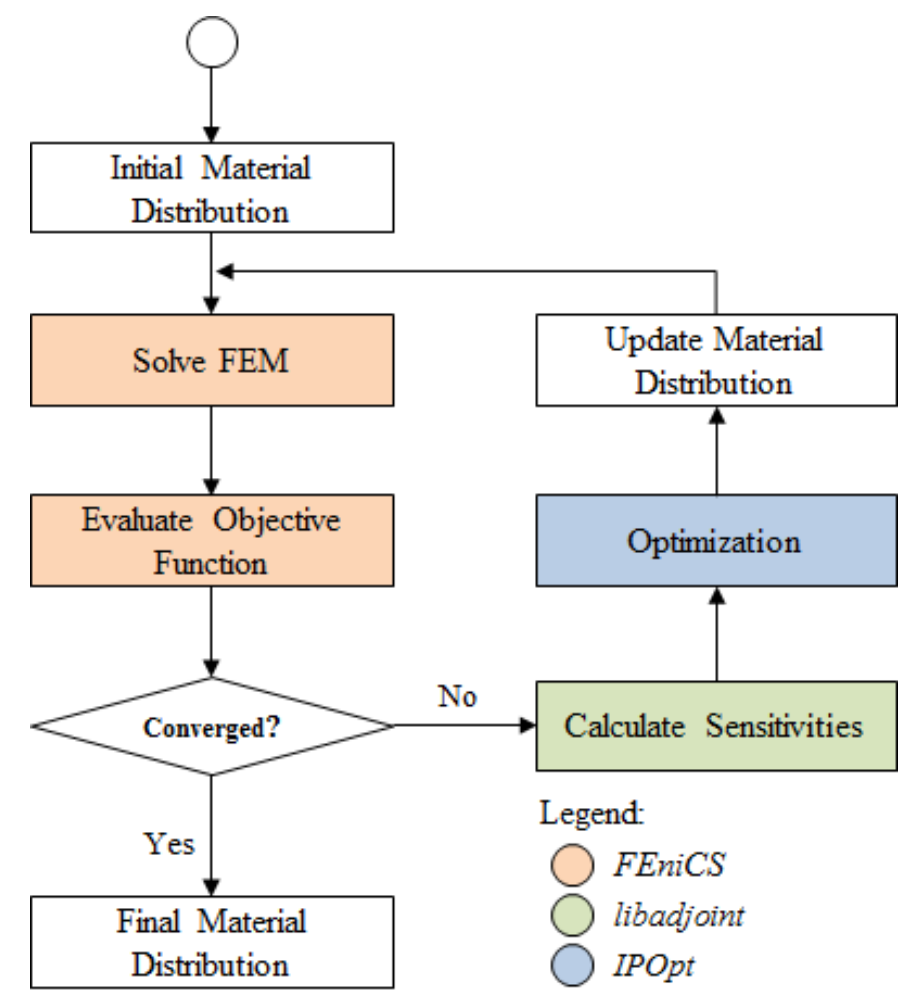

\subsubsection{Implementation of Flow Machine Rotor Optimization}

The sequence of steps to solve the optimization involves firstly defining an initial material distribution and the boundary conditions, then the FEniCS routines are called to solve the FEM system returning the solution vector $\left[\mathbf{u}_{1} \mathbf{u}_{2} \mathbf{p}\right]$. With this, the functional is evaluated and the dolfin-adjoint is called and performs the adjoint problem derivation, in order to calculate the sensitivity of the functional with respect to the design variable. Next, this information is passed to the optimizer IPOpt, that computes the next material distribution over the domain. The process is repeated until the functional value converges, in which case the optimum solution is reached. Figure 5.2 shows the steps cited above.

The FEniCS has an user-friendly interface, as cited above, where the implementation of the weak form problem can be done in a language similar to the math syntax. Thus, the implementation of Navier-Stokes in a rotating reference system equations (Eq. 2.4) can be done as shown in Listing 5.1 . 


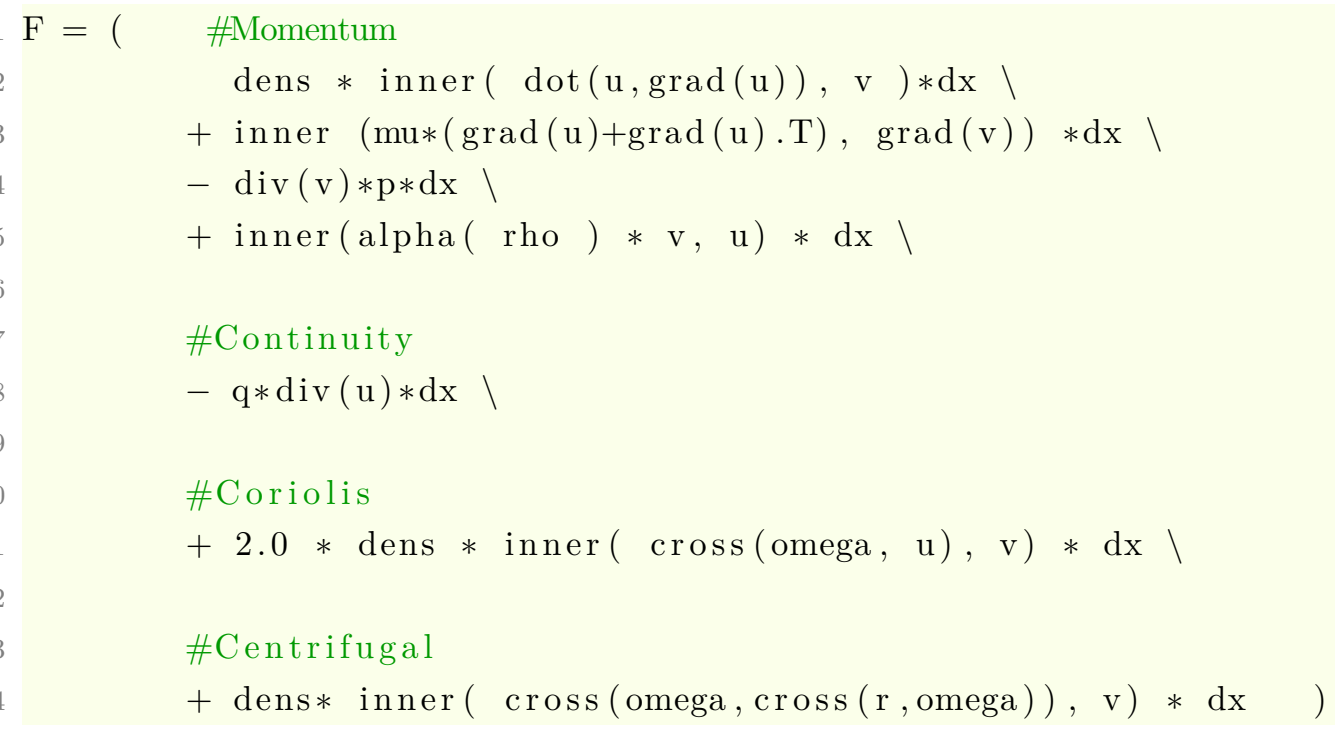

Listing 5.1 - Variational problem in FEniCS notation. 


\section{NUMERICAL RESULTS}

The following sections present the results obtained by using the methodology presented in Chapter 5. Some optimized pump topologies generated are shown and compared with the literature results of Romero and Silva (2014). Also in Section 6.2 some results for turbine optimization are shown.

Aiming to verify the effectiveness of the FEM algorithm, the FE results, implemented in the FEniCS environment, are compared with the results obtained with the commercial software $A N S Y S$ for different geometries. This comparison is presented in Appendix A and a qualitative analysis is performed.

An analysis of the influence of the volute considering the straight blade rotor is presented in Section 6.3. In which the pump characteristics, such as pressure head, energy dissipation, vorticity and torque, are evaluated to define the necessity of having, or not, a volute on the flow machine.

\subsection{Topology Optimization Results}

The topology optimization method is used by considering only the flow field between blades and without the volute influence. Even though the fluid in a real flow machine is three-dimensional, for the case of radial centrifugal impellers, the axial velocity component can be neglected in comparison to the radial and tangential components, hence the flow path can be approximated as a two-dimensional problem (ROMERO; SILVA, 2014).

The rotor is modelled as a half-circumference, given that the rotor has a radial symmetry, with the blade geometry being repeated in a radial pattern. Thus, for all cases shown in next sections, the design domain and boundary conditions presented in Figure 6.1 are used. The problem is solved by using arbitrary dimensions, thus, the domain $\Omega$ has an inner radius of 0.4 and an outer radius of 1.0. The fluid properties used are density equal to 1.0 and viscosity equal to 0.1 . The angular velocity $(\omega)$ used is $1000 \mathrm{rpm}$, unless specified other value. Also, the values for $k_{\max }$ and $k_{\text {min }}$, in Equation 3.1, are 10,000 and 0, respectively. These normalized properties are used in this work in order to avoid numerical issues, however, the optimization with real properties should be investigated in future works.

The following sections show the topology optimization results for different initial guesses, such as straight blade and the involute blade. In all following figures, the orange regions represent the fluid domain $(\alpha=1)$ and the blue regions represent the solid region $(\alpha=0)$. 
Figure 6.1 - Design domain and boundary conditions.

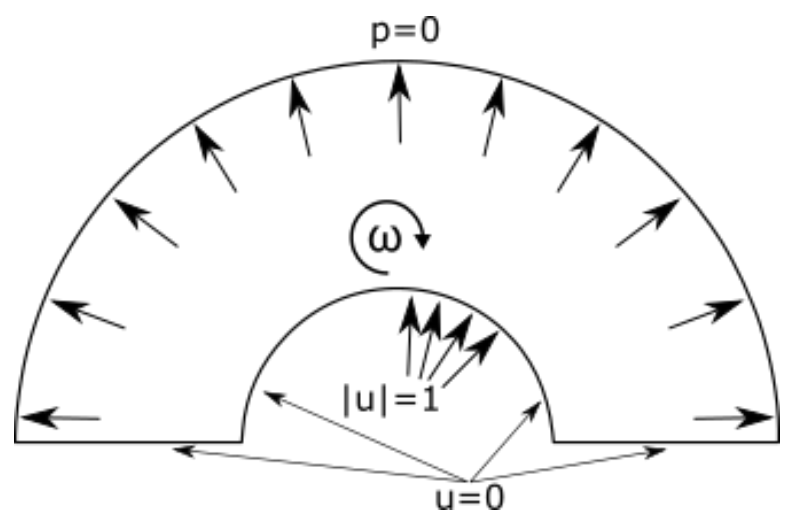

\subsubsection{Straight Blade Initial Guess}

Given the domain shown in Figure 6.1, it is possible to choose different initial conditions for the optimization process. Thus, in this section an initial domain distribution of a straight blade is used, as shown in Figure 6.2a, where the orange part represents fluid domain $(\alpha=1)$ and the blue part represents the solid domain $(\alpha=0)$. The velocity and pressure fields are shown in Figures 6.2b and 6.2c, respectively. It is worth to mention that the pressure field is calculated and plotted over all the domain due to the fact that the blue region is a porous domain and not a perfect solid.

Figure 6.2 - Straight blade initial guess.

(a) Initial domain.

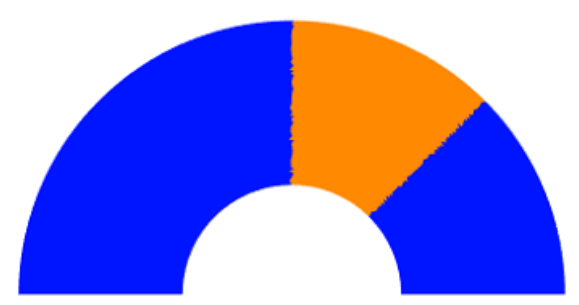

(b) Relative velocity field.

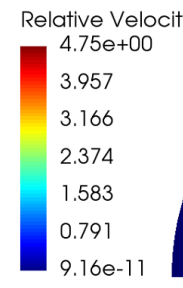

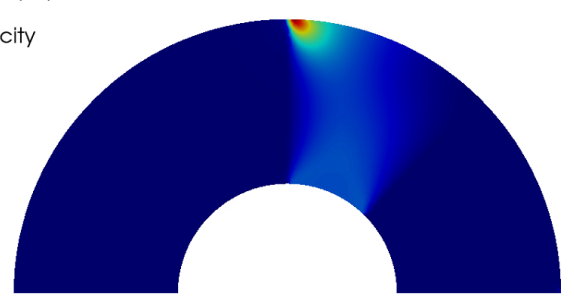

(c) Pressure field.

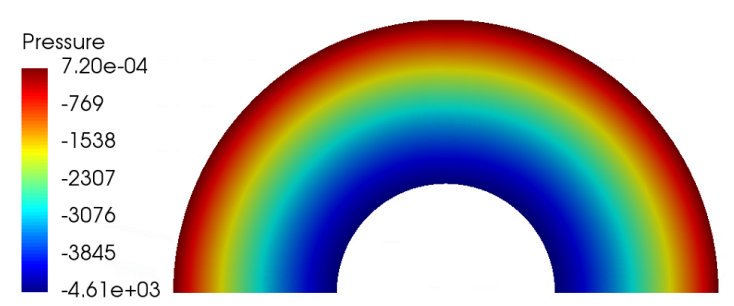

The topology optimization results considering Equation 3.16 with different values for the weight factors are shown in Figure 6.3. The corresponding rotor for each case is obtained by distributing the topologies over the rotor aiming to obtain the maximum number of blades without overlap, the rotors are shown in Figure 6.5. The velocity distribution for each blade is shown in Figure 6.4, the pressure distributions are very similar to the original 
blade (Fig.6.2c), thus they are not shown. The convergence curve for the optimization with energy dissipation functional only is shown in Figure 6.6, the curves for other cases is very similar, thus they are not show. The value for each functional, energy dissipation, vorticity and power, for each result is shown in Table 6.1. The results show that there is a Pareto relation between the functionals, i.e., one functional improves in detriment to other. For example, the case with energy and vorticity optimization $6.3 \mathrm{~b}$ ) has a better vorticity value (54.508), however, a worse energy dissipation (11.518), when compared with the case with energy dissipation only, which has an energy dissipation of 6.188 and vorticity of 65.339 .

The results show that the topology tends to grow around the geometry of the initial guess, given that the design variable is mainly distributed in the surrounds of the initial domain. The velocity profile indicates that regions with high porosity have velocities near to zero. The pressure distribution does not change much among the cases, indicating small pressure improvements. This can be observed in Table 6.1, in which the change in the pressure module is very small.

The result involving the energy functional as the only objective function (Figure 6.3a) imposes a division on the flow, which is very similar to the effect of adding splitters between the blades (GÖLCÜ; PANCAR; SEKMEN, 2006). This case has an improvement of the energy dissipation of $10.65 \%$ in comparison with the original blade.

The vorticity can be diminished by introducing regions with a porous domain, as it can be seen in Figure 6.3b, where the green region represents an intermediate material between fluid and solid. This also occurs in Figure 6.3d and both cases have a better value for the vorticity, showing improvements of $10.37 \%$ and $15.93 \%$, respectively.

Figure 6.3 - Topology optimization results by considering a straight blade as initial guess.

(a) $w_{e}=1.0, w_{v}=0.0, w_{p}=0.0$.

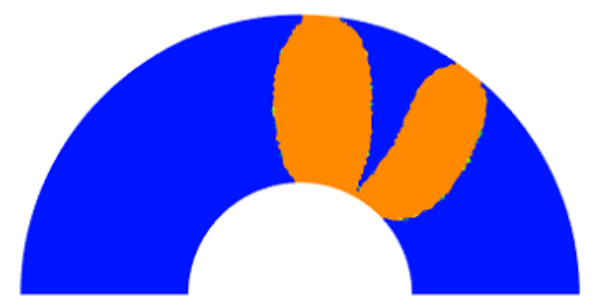

(c) $w_{e}=0.7, w_{v}=0.0, w_{p}=0.3$.

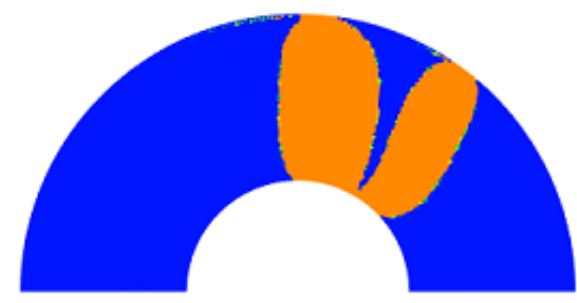

(b) $w_{e}=0.7, w_{v}=0.3, w_{p}=0.0$.

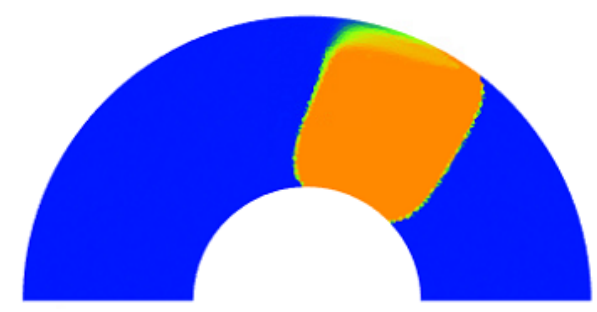

(d) $w_{e}=0.5, w_{v}=0.25, w_{p}=0.25$.

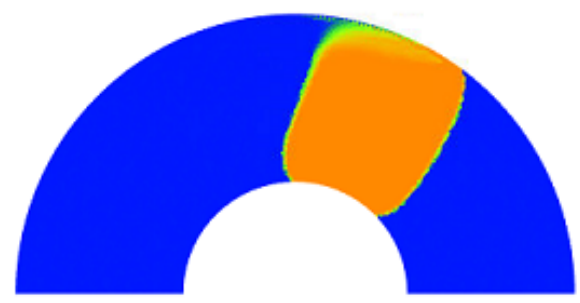


Figure 6.4 - Velocity fields for topology optimization results by considering a straight blade as initial guess.

(a) $w_{e}=1.0, w_{v}=0.0, w_{p}=0.0$.

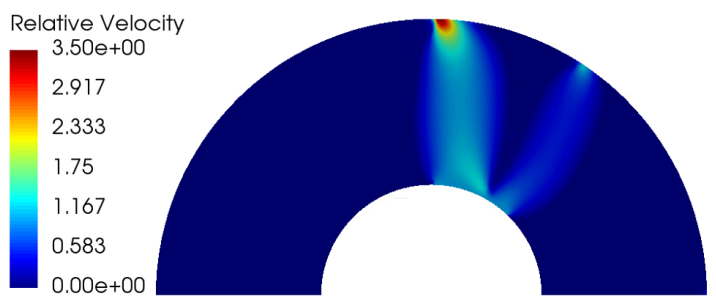

(c) $w_{e}=0.7, w_{v}=0.0, w_{p}=0.3$.

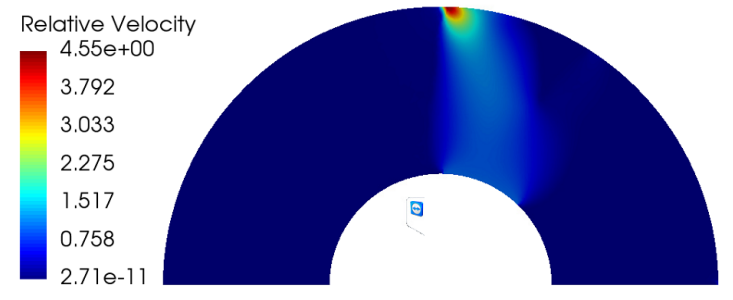

(b) $w_{e}=0.7, w_{v}=0.3, w_{p}=0.0$.

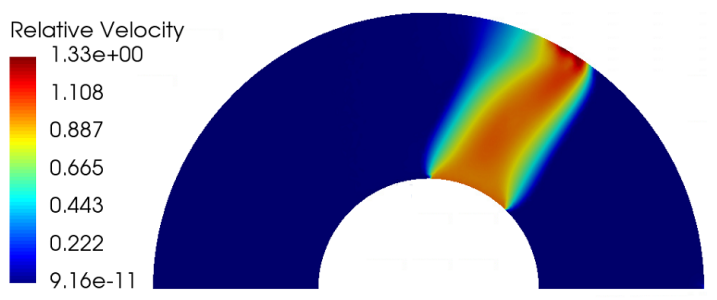

(d) $w_{e}=0.5, w_{v}=0.25, w_{p}=0.25$.

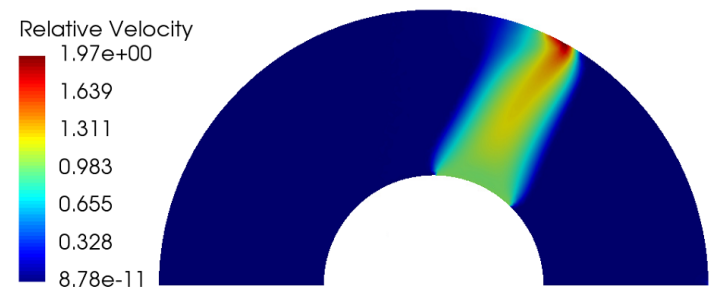

Figure 6.5 - Entire rotors corresponding to each topology optimization result by considering a straight blade as initial guess.

(a) $w_{e}=1.0, w_{v}=0.0, w_{p}=0.0$.

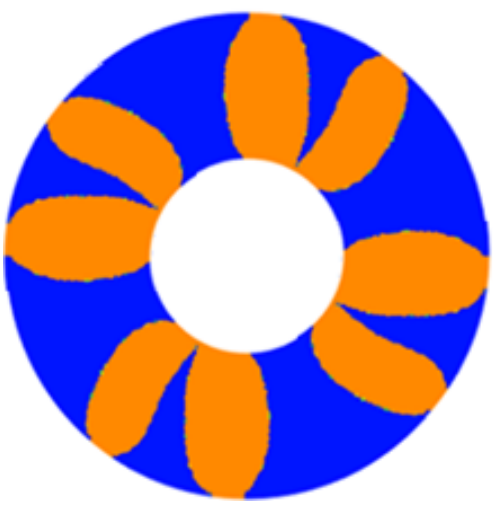

(c) $w_{e}=0.7, w_{v}=0.0, w_{p}=0.3$.

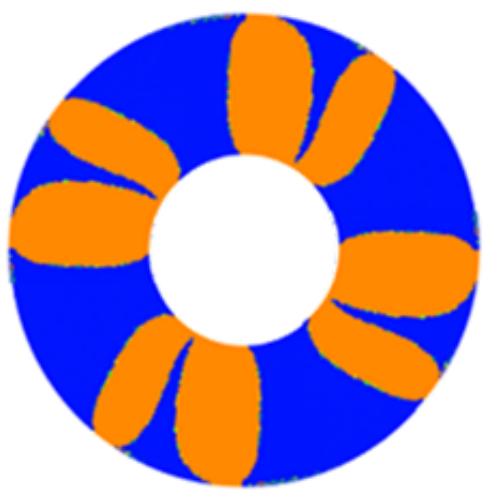

(b) $w_{e}=0.7, w_{v}=0.3, w_{p}=0.0$.

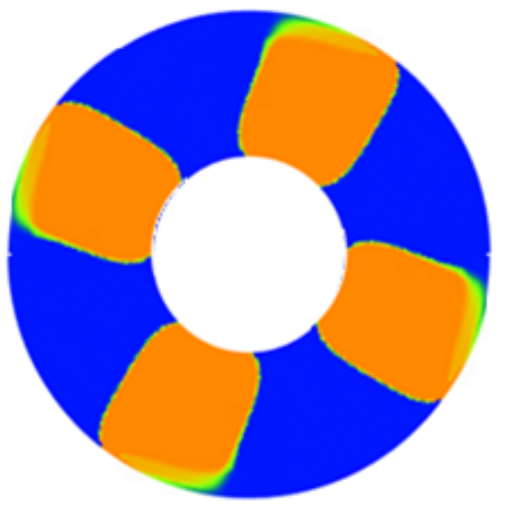

(d) $w_{e}=0.5, w_{v}=0.25, w_{p}=0.25$.

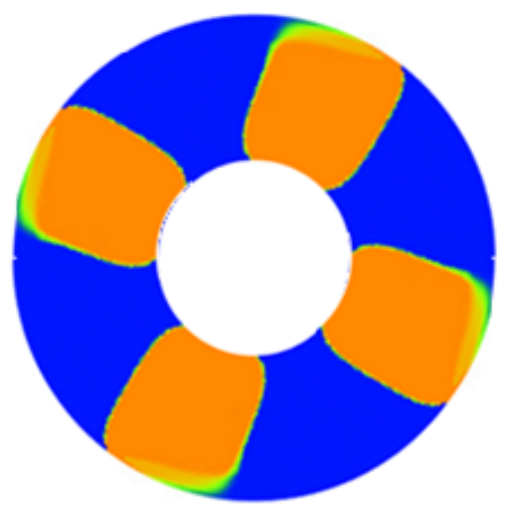


Figure 6.6 - Convergence curve for optimization with straight blade initial guess and $w_{e}=1$.

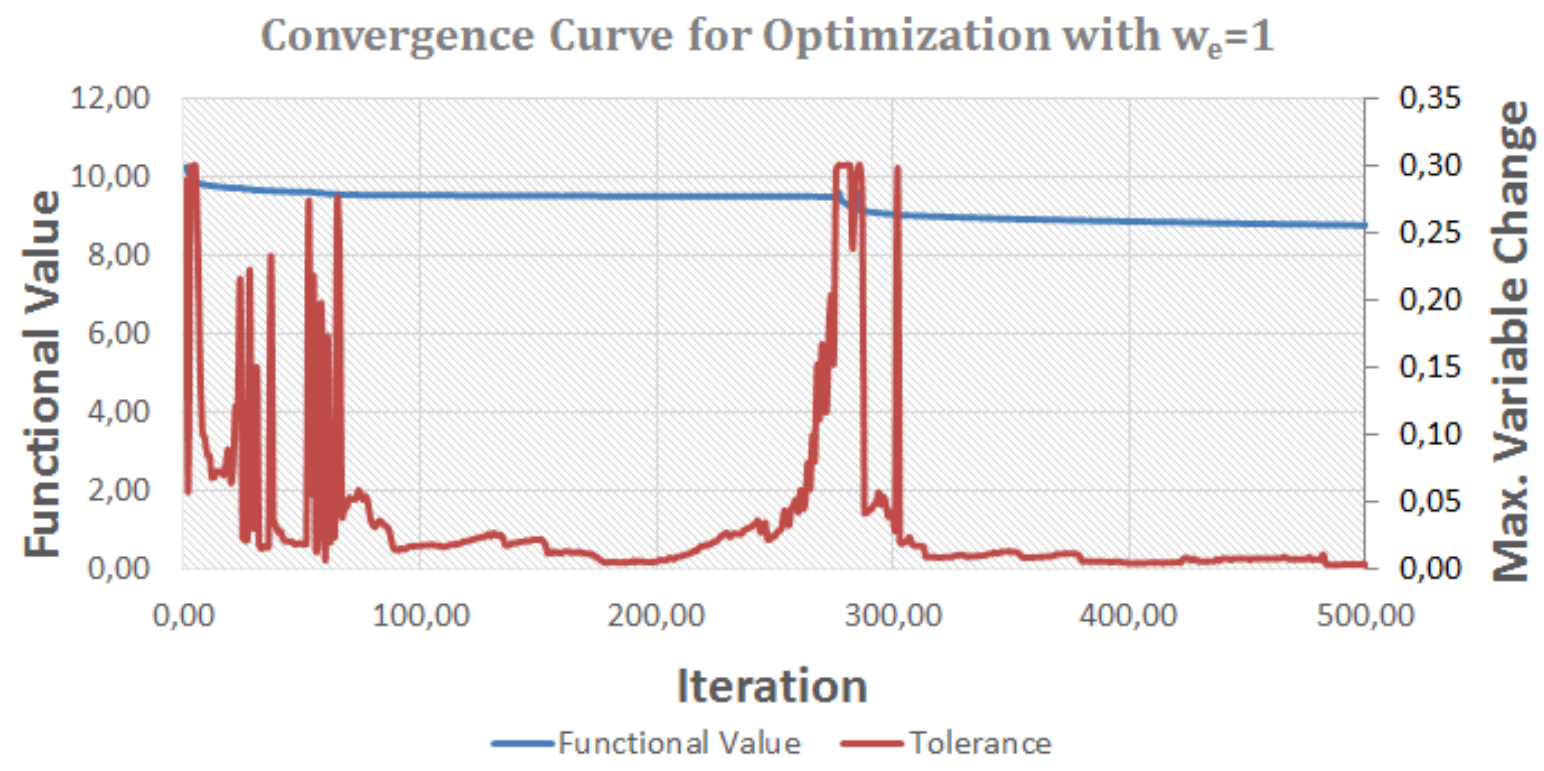

Table 6.1 - Functional values results by considering a straight blade as initial guess.

\begin{tabular}{|c|c|c|c|c|c|c|c|c|}
\hline & $w_{e}$ & $w_{v}$ & $w_{p}$ & $\begin{array}{c}\text { Volume } \\
(25 \%)\end{array}$ & $\begin{array}{l}\text { Pressure } \\
\text { (Inlet) }\end{array}$ & $\begin{array}{c}\text { Energy } \\
\text { Dissipation }\end{array}$ & Vorticity & Torque \\
\hline Straight blade & - & - & - & 0.34 & -4569.71 & 9.97 & 62.95 & 33.80 \\
\hline Figure $6.3 \mathrm{a}$ & 1.0 & - & - & 0.34 & -4578.24 & 8.59 & 60.09 & 28.40 \\
\hline Figure $6.3 \mathrm{~b}$ & 0.7 & 0.3 & - & 0.34 & -4571.80 & 11.50 & 8.27 & 28.72 \\
\hline Figure $6.3 \mathrm{c}$ & 0.7 & - & 0.3 & 0.34 & -4572.22 & 9.48 & 60.28 & 27.66 \\
\hline Figure $\overline{\mid \overline{6.3 \mathrm{~d}}}$ & 0.6 & 0.2 & 0.2 & 0.34 & -4568.15 & 12.56 & 10.20 & 27.73 \\
\hline
\end{tabular}

\subsubsection{Involute Blade Initial Guess}

Given the domain shown in Figure 6.1 and the initial distribution for the design variable of an involute blade, as shown in Figure 6.7a, the velocity and pressure fields shown in Figures 6.7b and 6.7c respectively, can be obtained. It is important to notice that Figure 6.7a the orange part represents the design variable with value 1 (fluid domain) and the blue part represents the design variable with value 0 (solid domain). 
Figure 6.7 - Involute blade optimization.

(a) Initial domain.

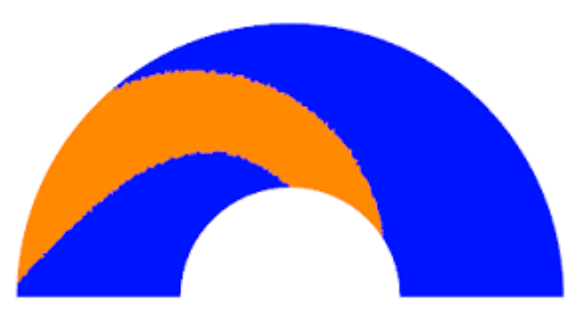

(b) Relative velocity field.

(c) Pressure field.

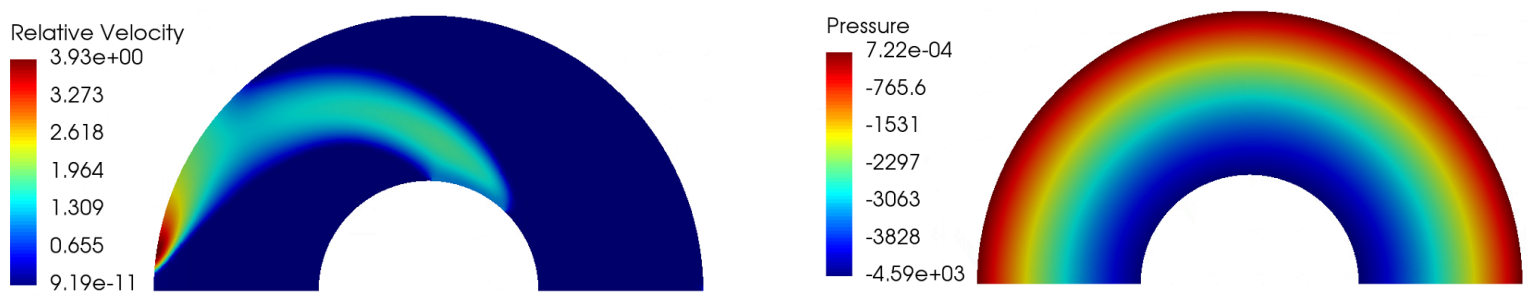

The topology optimization results considering Equation 3.16 with different values for the weight factors and a volume restriction of $1 / 3$ of the volume are shown in Figure 6.8 and the corresponding rotor for each case is shown in Figure 6.10. The velocity distribution for each blade is shown in Figure 6.9, the pressure distributions are very similar to the original involute blade (Fig.6.7c), thus they are not shown. The value for each functional, energy dissipation, vorticity and power, for each result is shown in Table 6.2.

The results show that the final topology is influenced by the different initial guess, given that the final topologies are different from the obtained before. The topologies obtained for the energy dissipation minimization only (Fig.6.8a) and for the minimization of energy dissipation and torque (Fig 6.8c) are very similar.

The results show, again, that there is a Pareto relation among the functionals, where the improvement of a objective function occurs in detriment of another. For example, the case with energy and vorticity optimization (6.8b has a better vorticity value (10.19) however a worse energy dissipation (36.49), when compared with the case designed with energy dissipation only, that has an energy dissipation of 11.78 and vorticity of 84.33 . 
Figure 6.8 - Optimization results by considering a involute blade as initial guess.

(a) $w_{e}=1.0, w_{v}=0.0, w_{p}=0.0$.

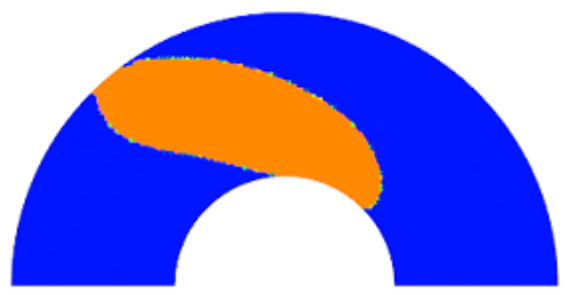

(c) $w_{e}=0.5, w_{v}=0.0, w_{p}=0.5$.

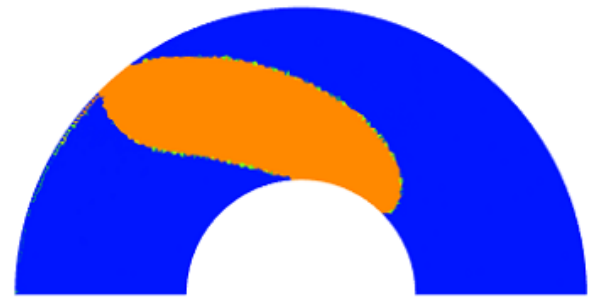

(b) $w_{e}=0.7, w_{v}=0.3, w_{p}=0.0$.

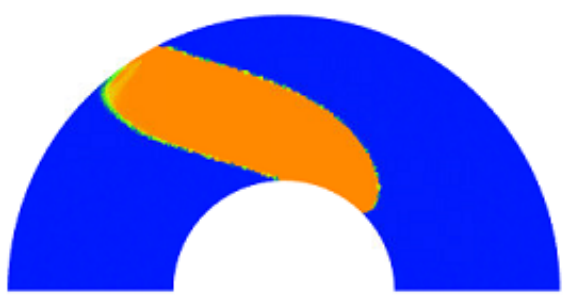

(d) $w_{e}=0.5, w_{v}=0.25, w_{p}=0.25$.

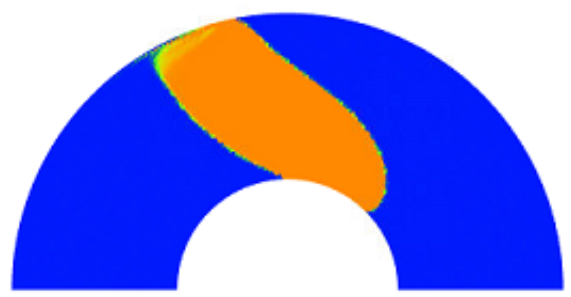

Table 6.2 - Functional values results by considering a involute blade as initial guess.

\begin{tabular}{|c|c|c|c|c|c|c|c|}
\hline & $w_{e}$ & $w_{v}$ & $w_{p}$ & Volume & Energy dissipation & Vorticity & Torque \\
\hline Involute blade & - & - & - & 0.38 & 14.01 & 130.77 & 33.94 \\
\hline Figure $6.8 \mathrm{a}$ & 1.0 & - & - & 0.44 & 11.78 & 84.33 & 34.22 \\
\hline Figure $6.8 \mathrm{~b}$ & 0.7 & 0.3 & - & 0.44 & 36.49 & 10.19 & 33.27 \\
\hline Figure $\overline{6.8 \mathrm{c}}$ & 0.5 & - & 0.5 & 0.44 & 12.02 & 84.28 & 33.66 \\
\hline Figure $\overrightarrow{\overline{6.8 \mathrm{~d}}}$ & 0.5 & 0.25 & 0.25 & 0.44 & 14.86 & 14.93 & 33.50 \\
\hline
\end{tabular}

Figure 6.9 - Velocity fields for topology optimization results by considering a involute blade as initial guess.
(a) $w_{e}=1.0, w_{v}=0.0, w_{p}=0.0$.

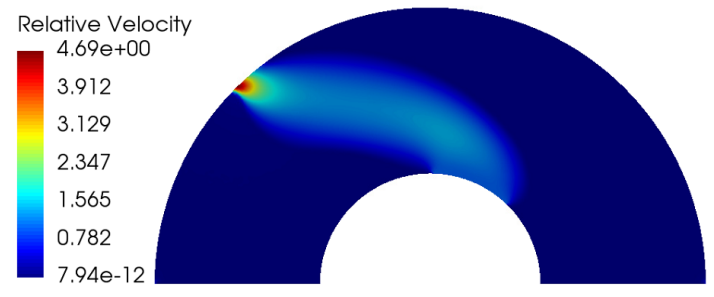

(c) $w_{e}=0.5, w_{v}=0.0, w_{p}=0.5$.

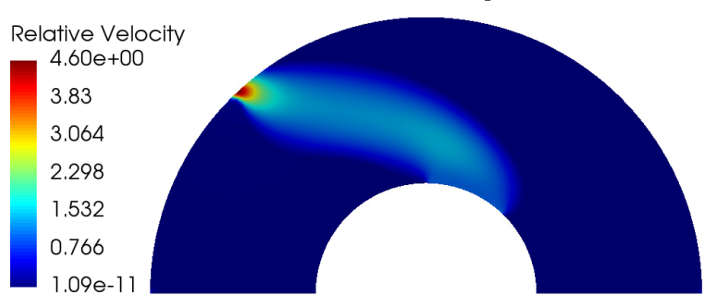

(b) $w_{e}=0.7, w_{v}=0.3, w_{p}=0.0$.

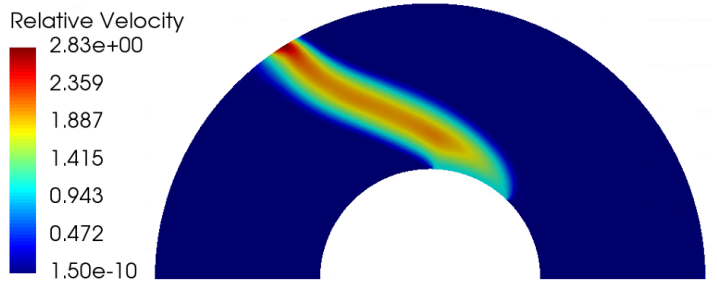

(d) $w_{e}=0.5, w_{v}=0.25, w_{p}=0.25$.

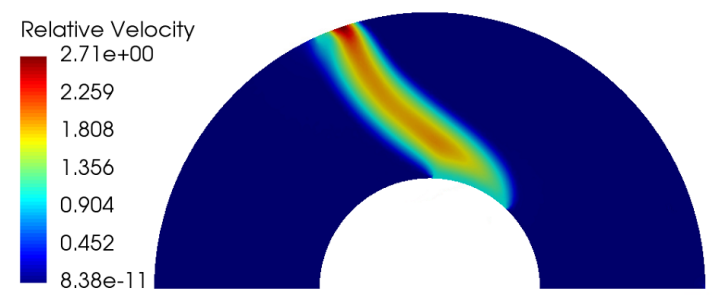


Figure 6.10 - Entire rotors corresponding to each topology optimization result by considering a involute blade as initial guess.

(a) $w_{e}=1.0, w_{v}=0.0, w_{p}=0.0$.

(b) $w_{e}=0.7, w_{v}=0.3, w_{p}=0.0$.
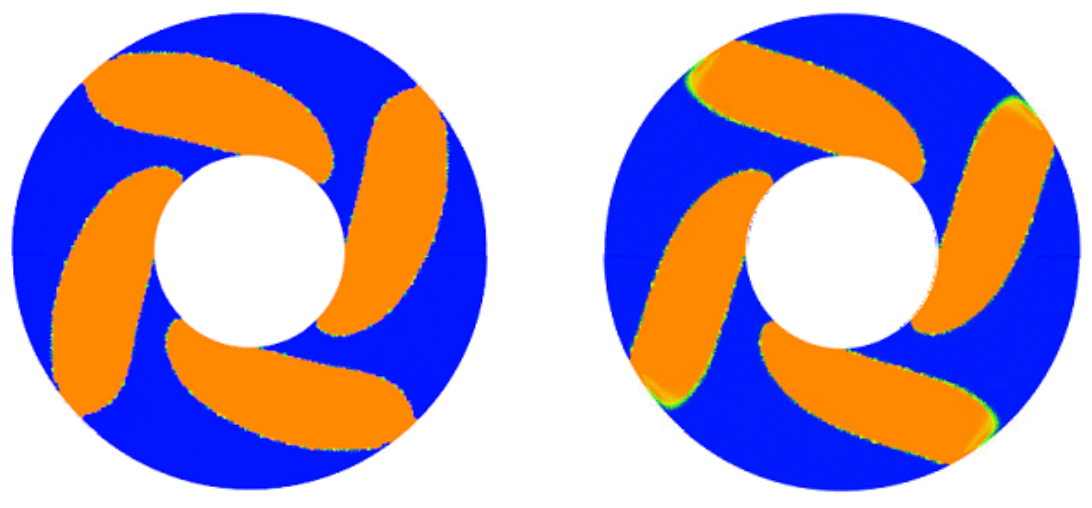

(c) $w_{e}=0.5, w_{v}=0.0, w_{p}=0.5$.

(d) $w_{e}=0.5, w_{v}=0.25, w_{p}=$ 0.25 .
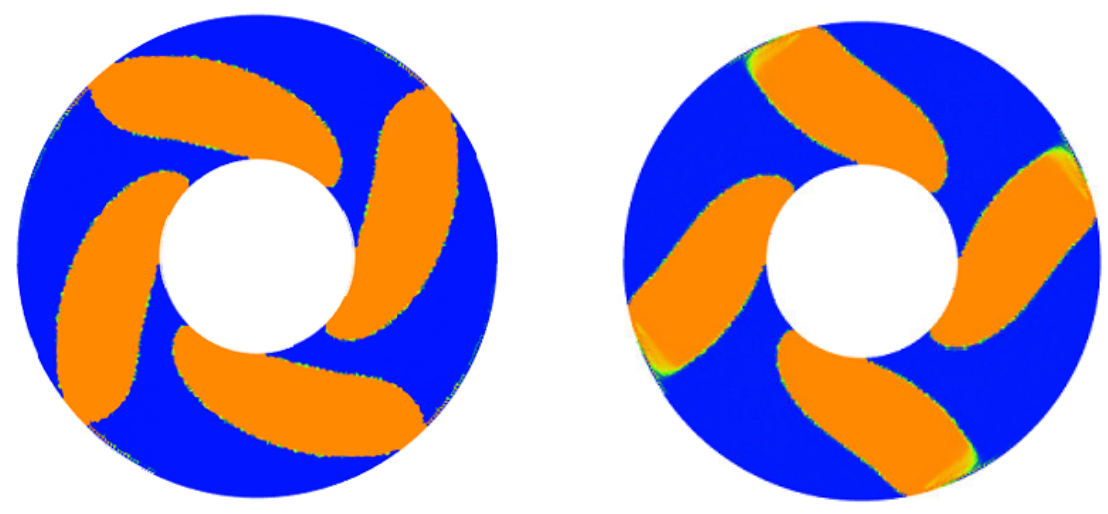


\subsection{Topology Results for Turbine}

The topology optimization method is used by considering only the flow field between blades and without the volute influence. The rotor is modelled as a half-circumference, given that the rotor has a radial symmetry, with the blade geometry being repeated in a radial pattern. The design domain and boundary conditions presented in Figure 6.11.

Again, the problem is solved by using arbitrary dimensions, thus, the domain $\Omega$ has an inner radius of 0.4 and an outer radius of 1.0. The fluid properties used are density equal to 1.0 and viscosity equal to 0.1 . The angular velocities $(\omega)$ used are $20 \mathrm{rpm}$ and $100 \mathrm{rpm}$.

Figure 6.11 - Design domain and boundary conditions for turbine.

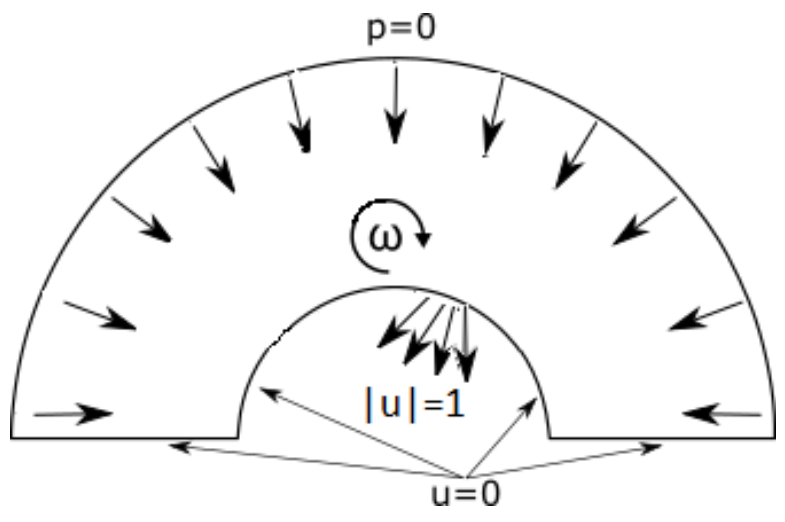

\subsubsection{Involute Blade Initial Guess}

Given the domain shown in Figure 6.11 and the initial distribution for the design variable of an involute blade, as shown in Figure 6.12, the velocity and pressure fields shown in Figures 6.13 and 6.14, respectively, can be obtained for both rotations (20 and $100[\mathrm{rpm}])$. It is important to notice that Figure 6.12 the orange part represents the design variable with value 1 (fluid domain) and the blue part represents the design variable with value 0 (solid domain).

Figure 6.12 - Involute blade optimization Initial domain.

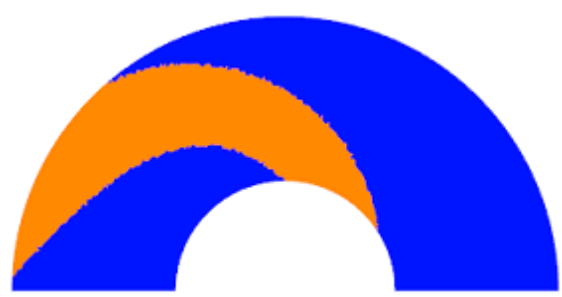

The pressure fields indicate that for low rotations the solid - fluid interaction at the blade contour causes a local increase in the pressure at these points. This is observed by comparing Figures 6.14a and 6.14b the effect of this local increase is more pronounced at the rotation of $20[\mathrm{rpm}]$. 
Figure 6.13 - Velocity fields for turbine initial guess with rotations: (a) $20[\mathrm{rpm}]$ and (b) $100[\mathrm{rpm}]$.

(a) $20 \mathrm{rpm}$.

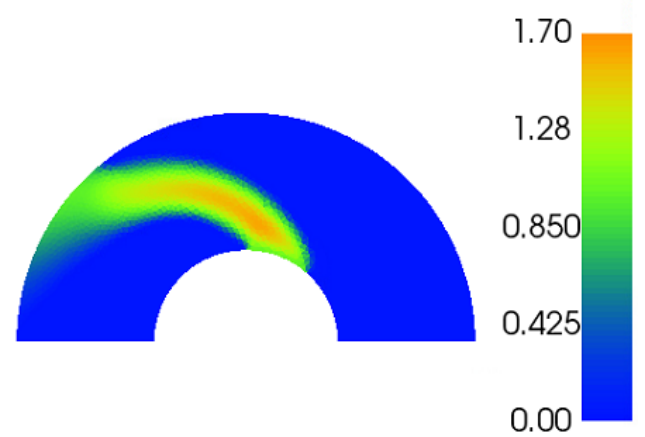

(b) $100 \mathrm{rpm}$.

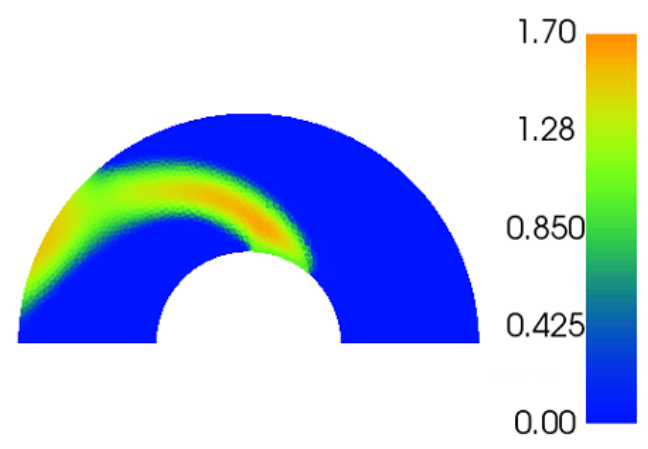

Figure 6.14 - Pressure fields for turbine initial guess with rotations: (a) 20[rpm] and (b) $100[\mathrm{rpm}]$.

(a) $20 \mathrm{rpm}$.
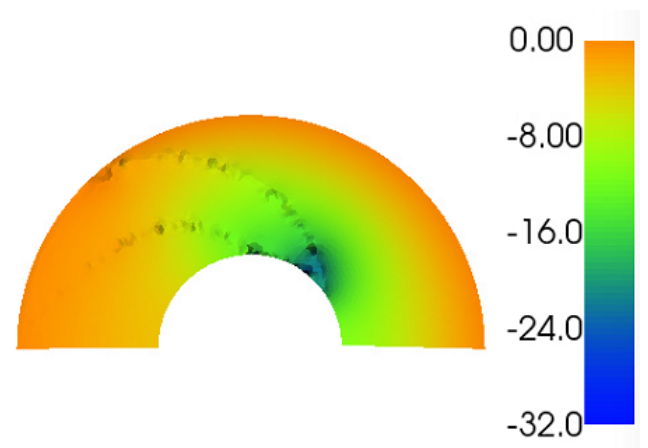

(b) $100 \mathrm{rpm}$.

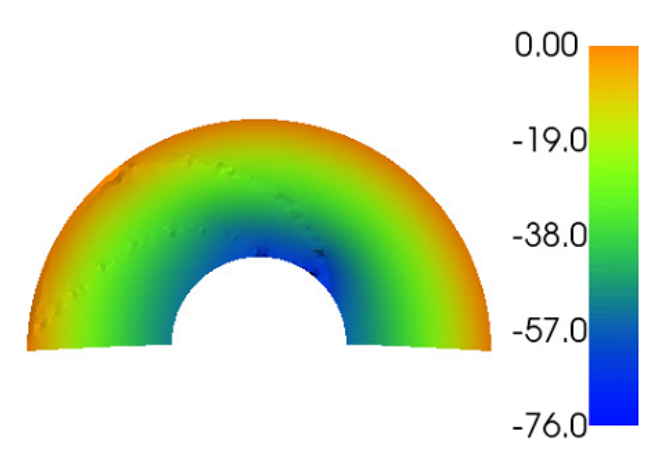

The topology optimization results considering only the power maximization function is shown in Figure 6.15. The respective entire rotor for each rotation is shown in Figure 6.18. The value for each functional, energy dissipation, vorticity and power, for each topology is shown in Table 6.3. The optimized topologies, again, are very similar to the ones of Romero and Silva (2014). Also, we observe that the final topologies are very influenced by the rotation amplitude.

Figure 6.15 - Topology optimization results by considering a involute blade as initial guess and power maximization with: (a) $20[\mathrm{rpm}]$ and (b) $100[\mathrm{rpm}]$.

(a) $20 \mathrm{rpm}$.

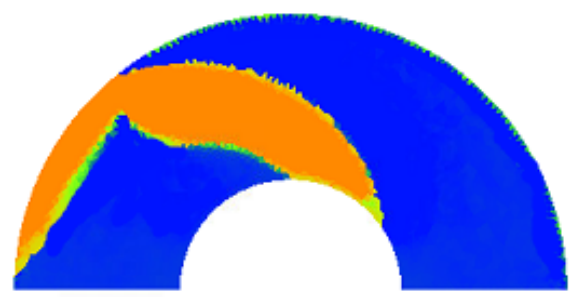

(b) $100 \mathrm{rpm}$.

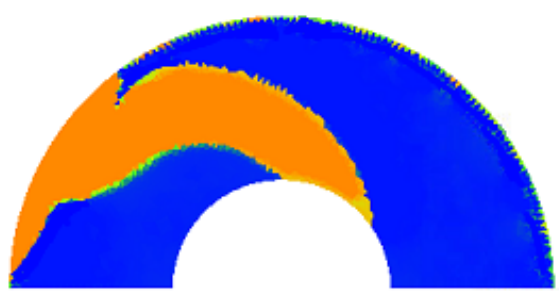


Table 6.3 show that the optimized topologies have a higher power generation than the initial involute blade for both rotations. However, they also show an increase in the functional values for energy dissipation and vorticity. The topology obtained with $20 \mathrm{rpm}$ has an increase of $8 \%$ in torque generation while the topology obtained with $100 \mathrm{rpm}$ has an increase of $2.7 \%$.

Table 6.3 - Functional values results by considering a involute blade as initial guess and power maximization.

\begin{tabular}{lccccc}
\hline & $\omega[\mathrm{rpm}]$ & Volume & Energy dissipation & Vorticity & Torque \\
\hline Involute blade & 20 & 0.38 & 4.36 & 38.23 & 0.75 \\
Figure 6.15a & 20 & 0.41 & 5.75 & 46.22 & 0.81 \\
Involute blade & 100 & 0.38 & 4.98 & 43.17 & 3.26 \\
Figure 6.15b & 100 & 0.44 & 5.43 & 42.05 & 3.35 \\
\hline
\end{tabular}

Figure 6.16 - Velocity fields for turbine results by considering a involute blade as initial guess and power maximization with: (a) 20[rpm] and (b) 100[rpm].
(a) $20 \mathrm{rpm}$.
(b) $100 \mathrm{rpm}$.
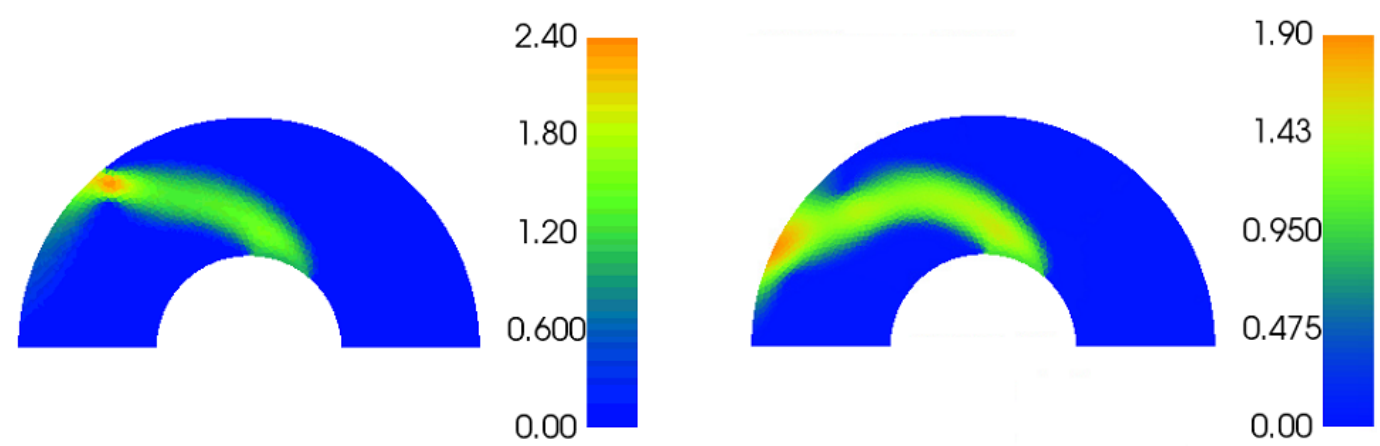

Figure 6.17 - Pressure fields for turbine results by considering a involute blade as initial guess and power maximization with: (a) $20[\mathrm{rpm}]$ and (b) $100[\mathrm{rpm}]$.

(a) $20 \mathrm{rpm}$.
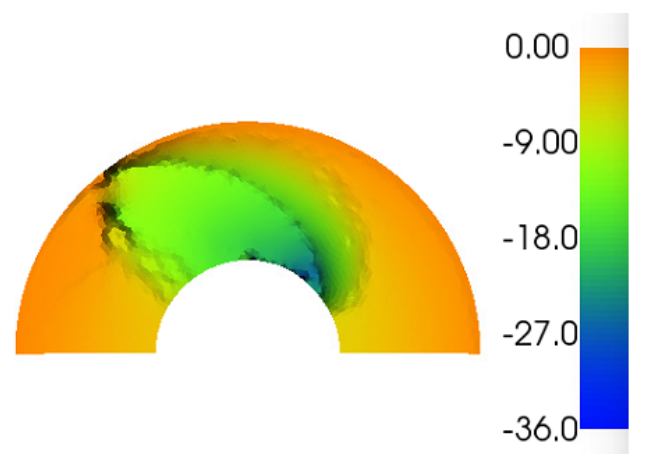

(b) $100 \mathrm{rpm}$.

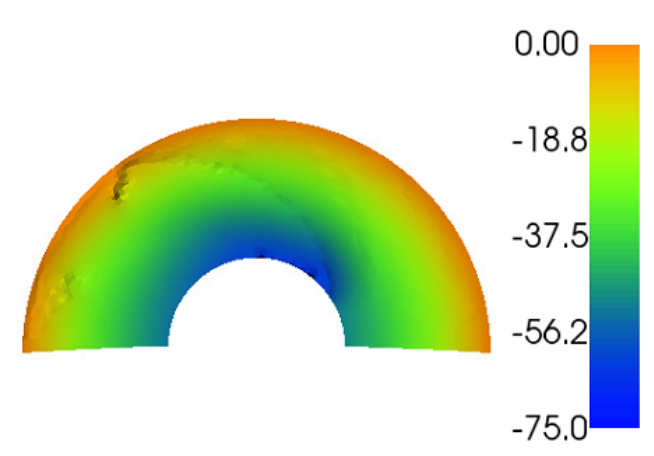


Figure 6.18 - Entire rotor for turbine results by considering a involute blade as initial guess and power maximization with: (a) $20[\mathrm{rpm}]$ and (b) $100[\mathrm{rpm}]$.

(a) $20 \mathrm{rpm}$.

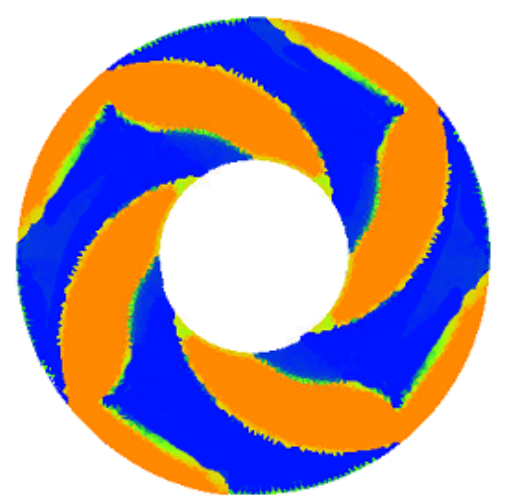

(b) $100 \mathrm{rpm}$.

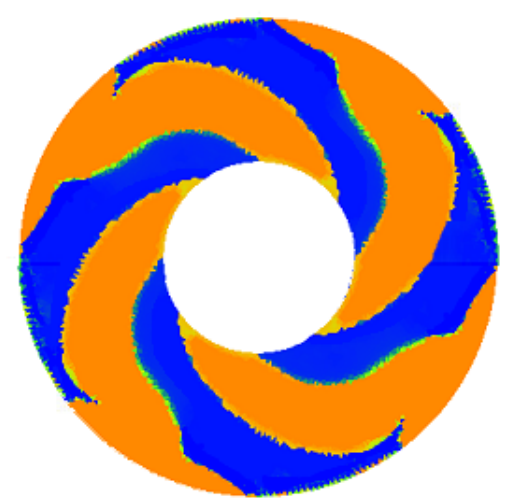

\subsection{Volute Simulation Analysis}

This work main objective is the optimization of the rotor of flow machine, however, other parts such as the volute can have a great influence on the overall performance of these machine. Thus, this chapter presents a brief analysis of the impact that the presence, or absence, of the volute causes in the machine operation.

The modeling is done by using the software ANSYS. The geometry is composed of a straight blade rotor, formed by six blades, and uses water as fluid (Table 6.4). The dimensions are based on small scale pumps, such as ventricular assist pumps. Two models are developed, the first one has a case without the volute, as presented in Figures 6.19c and 6.19d, and other has the case with the volute, as shown on Figures 6.19e and 6.19f.

Table 6.4 - Fluid Properties.

\begin{tabular}{ccc}
\hline Material & Density $\left[\mathrm{kg} / \mathrm{m}^{3}\right]$ & Viscosity $\left[N \cdot \mathrm{s} / \mathrm{m}^{3}\right]$ \\
\hline Fluid 1 & 1.0 & 0.1 \\
Water & 997.0 & 0.001 \\
\hline
\end{tabular}

It is important to notice that in fluid analysis the geometry defined is for the fluid domain, i.e., the volume created represents the part occupied by fluid. In this case, the structures shown on Figure 6.19 are the water domain.

The boundary conditions in this case are null velocity on the walls, relative pressure equals to zero on the nozzle, and velocity normal to surface with module $0.23[\mathrm{~m} / \mathrm{s}]$ in the inner side of the blades, as shown in Figure 6.20a, Figure 6.20b. The green part has the rotation of $\omega=1000 \mathrm{rpm}$ while the grey part is stationary, thus, the interface between these parts have conditions of "frozen rotor". 
Figure 6.19 - Rotor Model (Fluid Domain) with a Case with and without Volute.

(a) Rotor Isometric View.

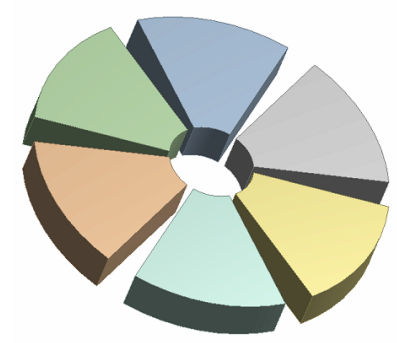

(c) Case without Volute - Isometric View.

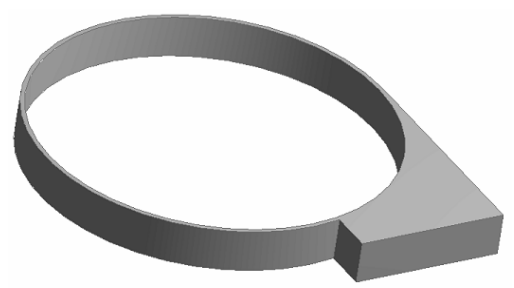

(e) Case with Volute - Isometric View.

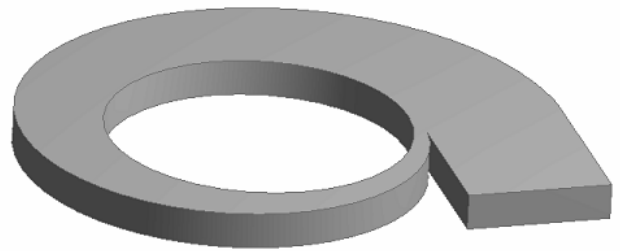

(b) Rotor Dimensions.

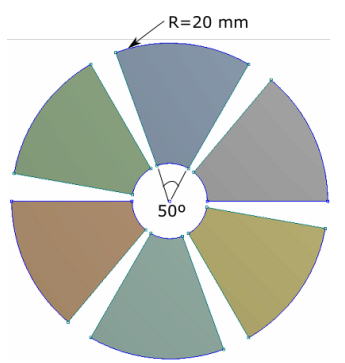

(d) Case without Volute Dimensions.

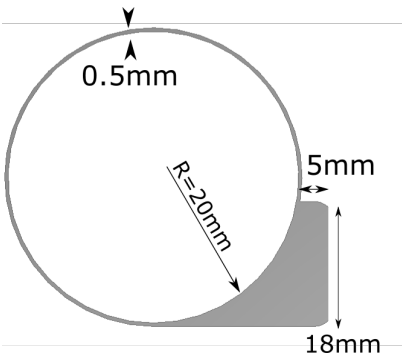

(f) Case with Volute Dimensions.

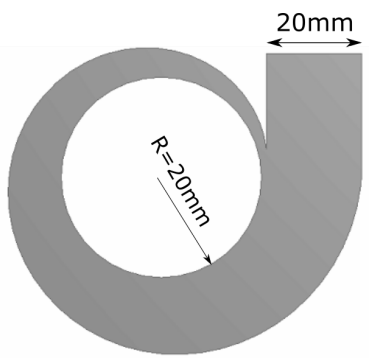

Figure 6.20 - Rotor Model with a Case with and without Volute Boundary Conditions.

(a) Rotor without Volute.

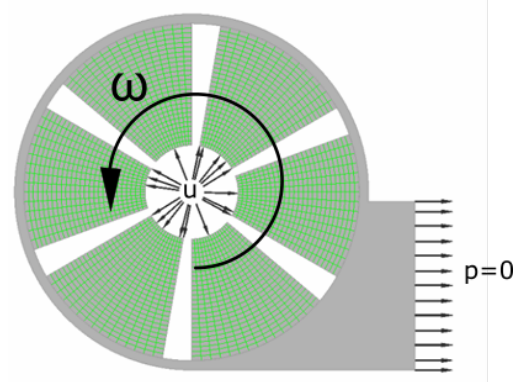

(b) Rotor with Volute.

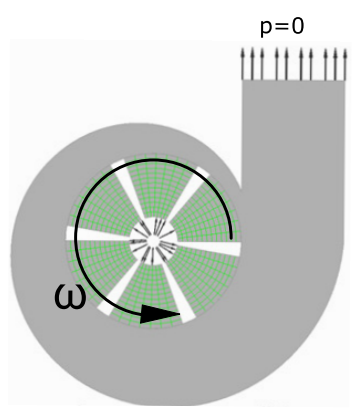

The absolute velocity and pressure fields, for both cases, are shown in Figures 6.21 and 6.22 . It is possible to notice that the stream lines for the case without volute (Fig.6.23a) shows vortex formation on almost all blades, while in the case with the volute (Fig 6.23b this effect is less pronounced. The case with the volute (Fig 6.22b) presents a more homogeneous pressure field and the stream lines are smoother. 
Figure 6.21 - Absolute Velocity and Pressure field for Case without the volute.

(a) Absolute Velocity Field (without volute).

(b) Pressure Field (without volute).
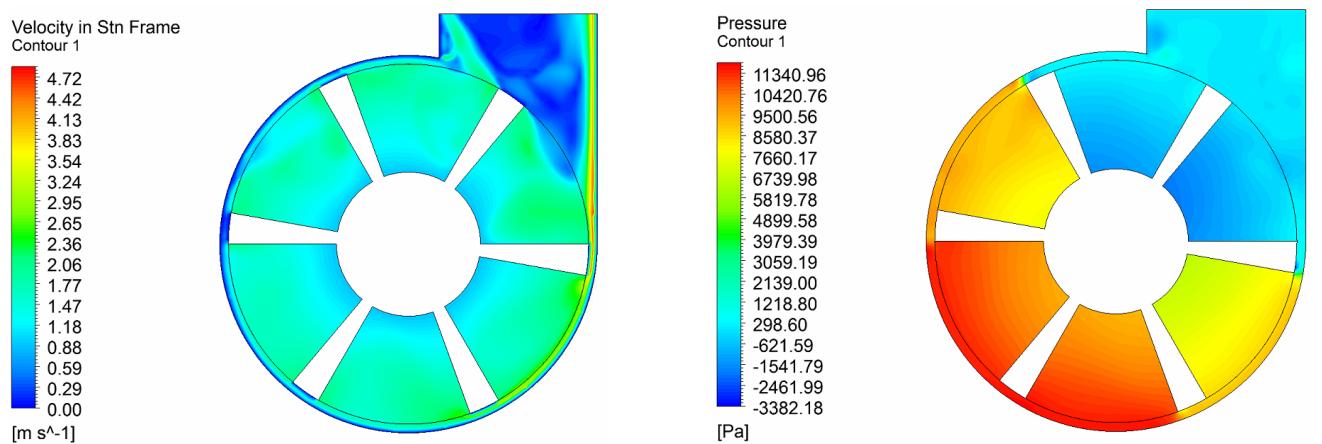

Figure 6.22 - Absolute Velocity and Pressure field for Case with the volute.

(a) Absolute Velocity Field (with volute).

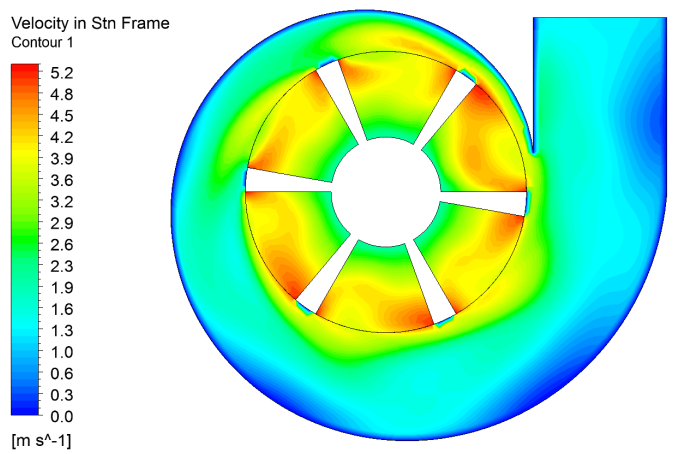

(b) Pressure Field (with volute).

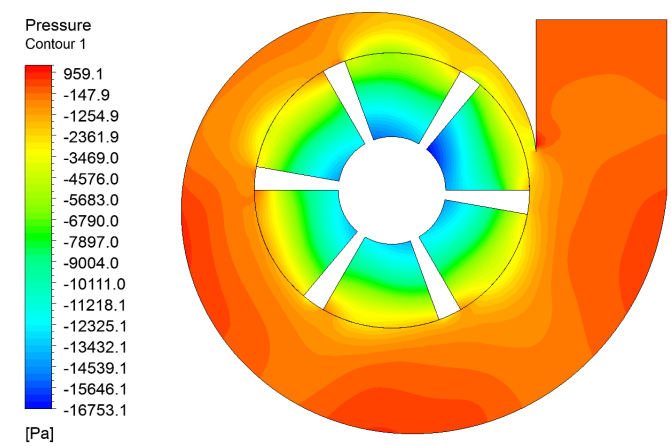

Figure 6.23 - Stream lines for both cases.

(a) Stream line for case without volute.

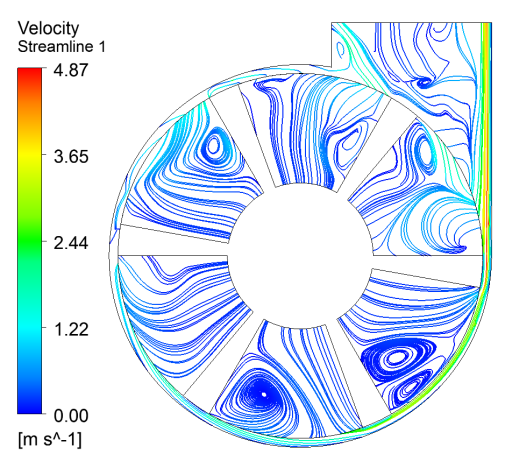

(b) Stream lines for case with volute.

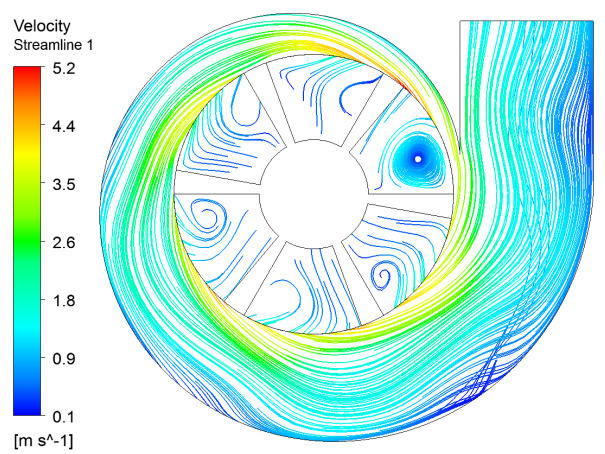

However, considering the pressure head for the pump, which is given by the difference between the pressure at the outlet and the minimum pressure at the inlet, the volute case has a lower pressure head. This occurs due to the fact that the fluid flows only through the gap between the rotor and the case (Fig. 6.21a), causing a increase in the local pressure in regions that do not connect directly with the nozzle (Fig. 6.24).

The simulation indicates that the pump with the volute has a velocity field with less 
recirculation (Fig, 6.23b) and a pressure field more homogeneous among the blades.

In the steady state, the dissipation of viscous energy is equivalent to the sum of the power held in the system by the external forces and variation of kinetic energy through the contour (OLESEN; OKKELS; BRUUS, 2006). That is, the pressure jump between the inlet and the outlet of the pump is related to the dissipation of energy (flow dissipation). Thus, the higher dissipation, the higher pressure jump. Hence, the energy dissipation is lower in the case with the volute.

The model used in the optimization considers that the flow behavior in the blades are similar to each other. Thus, only the case with the volute will represent well the simulations made with the single blade.

Figure 6.24 - Pressure Field (without volute).

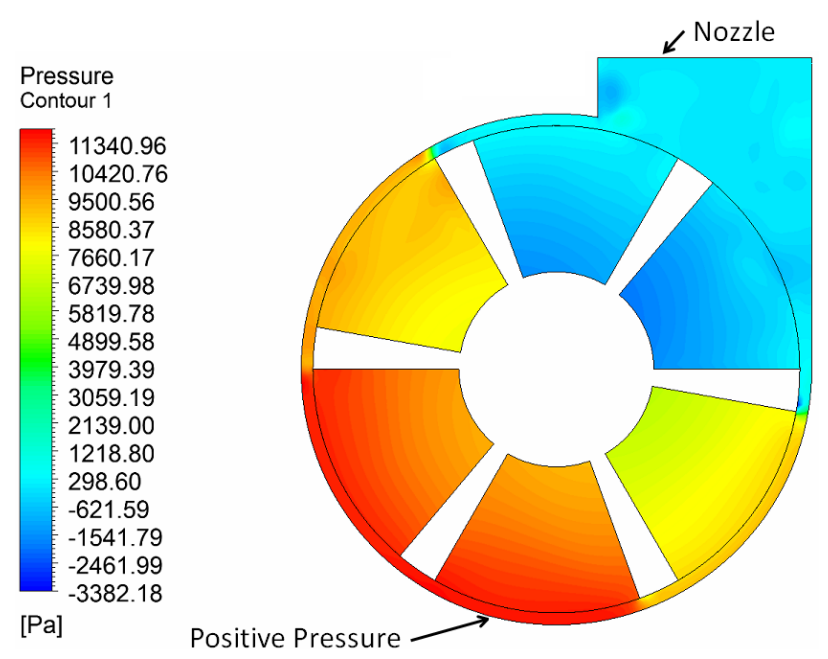




\section{EXPERIMENTAL METHODOLOGY}

This chapter presents the prototype design for a radial flow pump and also the experimental setup to perform the pump characterization. The prototype built is based on ventricular assist pumps, so it is a small scale pump. The characterization is made by measuring the pump output (pressure and mass flow).

\subsection{Pump Prototype Design}

The prototype is based on a ventricular assist pump, so its dimensions are smaller than the dimensions of industrial pumps. The machine consists essentially of three parts: the case, the motor and the rotor. These components are presented in the next sections.

\subsubsection{Pump Case Design}

The case is composed of a base with the outlet and a lid with the inlet. In order to evaluate the effects of the base in the fluid flow, two types of bases are proposed, one with a volute and other without a volute. These two models and the lid are shown in Figure 7.1

Figure 7.1 - Case models for prototypes.

(a) Model with Volute.

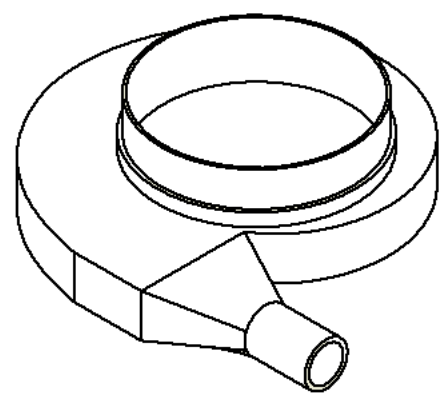

(b) Model without Volute.

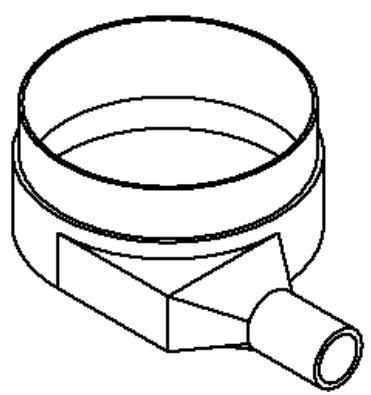

(c) Model Lid Inlet.

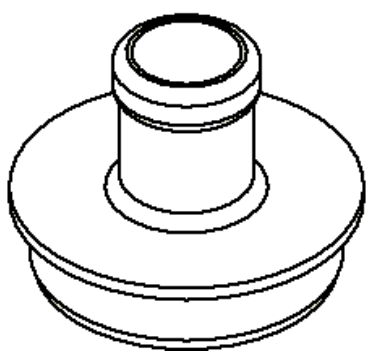

\subsubsection{Motor Design}

The motor consists of an electric brushless motor composed of six silicon steel rings with a coil (sixty turns) and a ring with embedded magnets to be coupled to the rotor. The pole change, on the motor, is done by using a controller and three hall sensors distributed $60^{\circ}$ apart of each other, as shown in Figure 7.2. The silicon steel rings and the ring of magnets are shown in Figure 7.3 . 
Figure 7.2 - Hall sensor position.

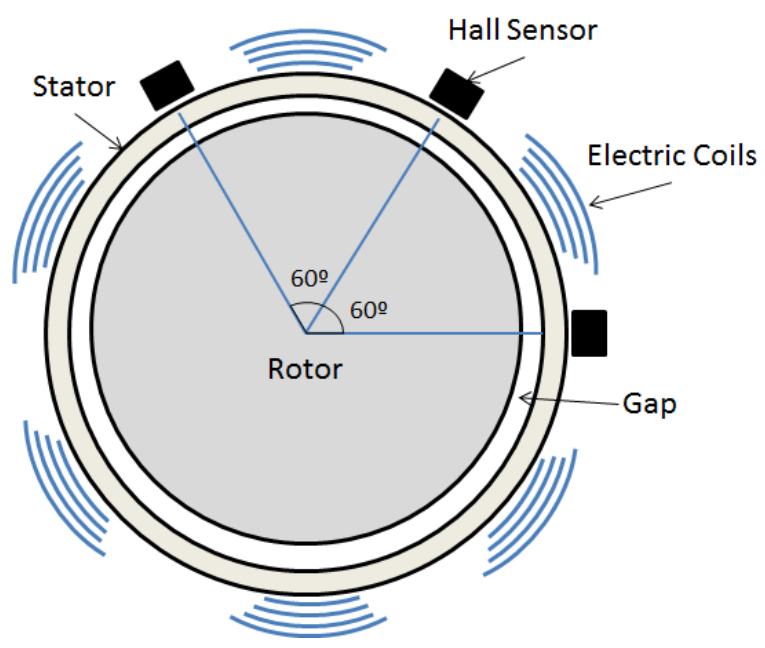

Figure 7.3 - Motor prototype.

(a) Stator: External Silicon Steel

Ring.

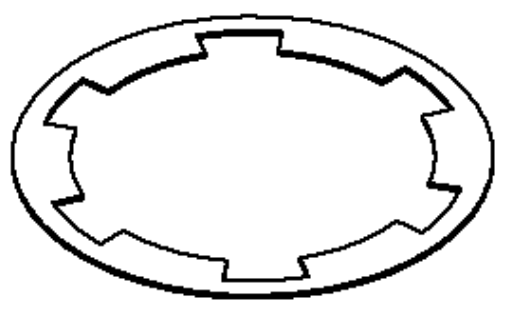

(b) Rotor: Ring of magnets.

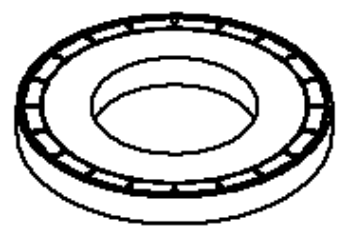

The ring of magnets is composed of a base material, in this case Polyvinyl chloride $(\mathrm{PVC})$, an internal iron ring and the magnets. The complete assemble is shown in Figure 7.4.

Figure 7.4 - Ring of magnets assemble.

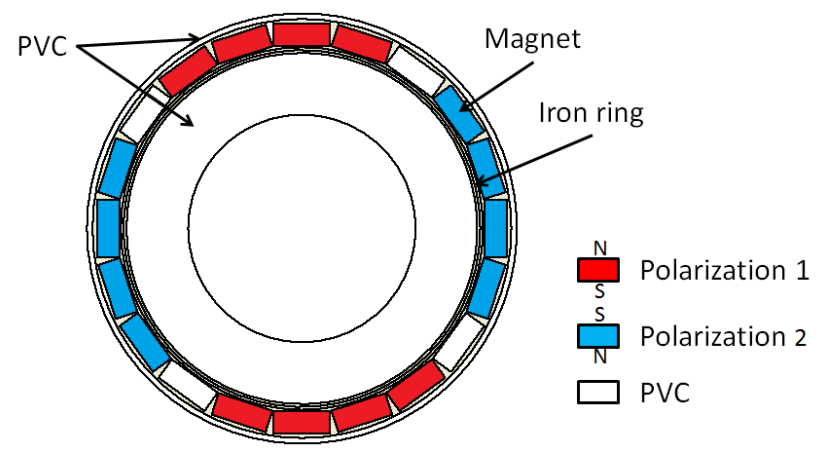

\subsubsection{Rotor Design}

The pump rotor (or impeller) is the focus of this optimization work, so many geometries are proposed for comparison purposes. The rotors initially proposed have six blades with different blade geometries for each rotor. The blades are also designed to follow the volute 
geometry, thus if the volute growths in a counter-clockwise fashion the rotor is designed to rotate counter-clockwise. The blades geometries are straight blades, curved blade and involute blade, as shown in Figure 7.5.

Figure 7.5 - Traditional rotor prototypes.

(a) Straight Blade Top view.

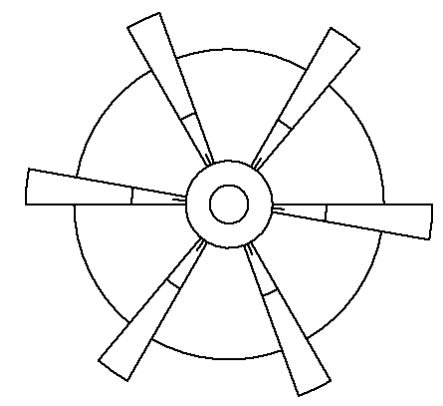

(d) Straight Blade Design.

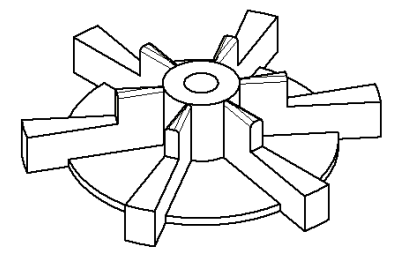

(b) Curved Blade Top view.

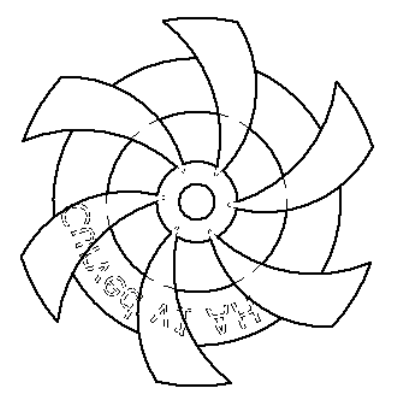

(e) Curved Blade Design.

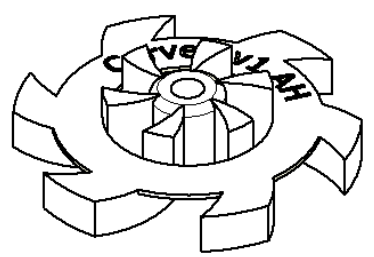

(c) Involute Blade Top view.

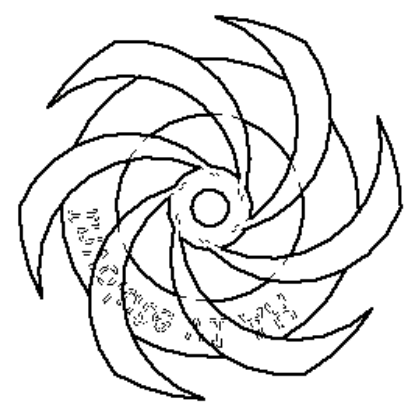

(f) Involute Blade Design.

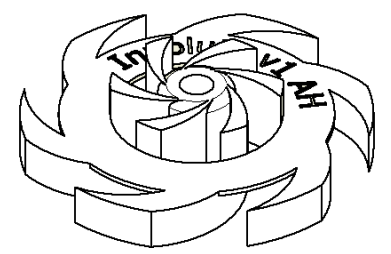

\subsubsection{Pump Design Assemble}

The complete pump assemble is shown in Figure 7.6 .

Figure 7.6 - Complete pump case assemble.

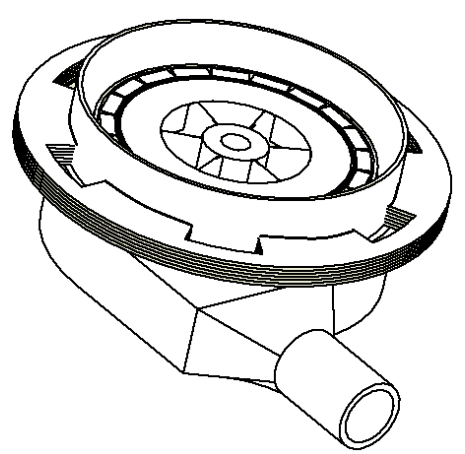

\subsection{Pump Prototype Manufacturing}

\subsubsection{D Printed Parts}

The prototype case, lid and rotors are built by using a 3D printer Stratasys Objet Connex350. The materials used are Stratasys commercial materials. Two types are used the translucent material VeroClear-RGD810, which grants a good dimensional stability, 
translucency and is rigid, and the opaque rigid material VeroWhitePlusRGD835 with similar properties. The case, lid and rotors are shown in Figure 7.7 .

Figure $7.7-3 \mathrm{D}$ printed parts.

(a) Case with volute.

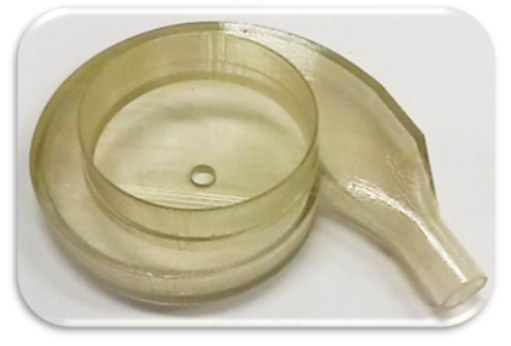

(d) Straight Blade Rotor.

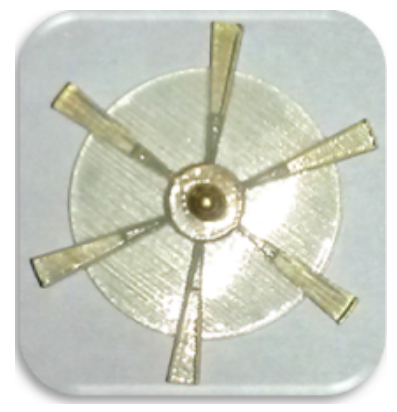

(b) Case without volute.

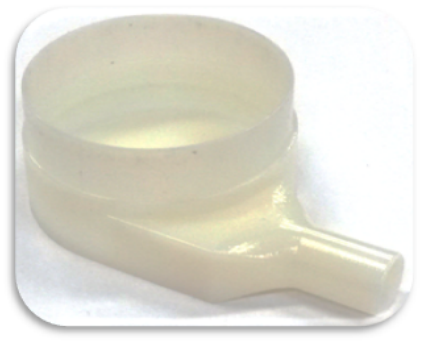

(e) Curved Blade Rotor.

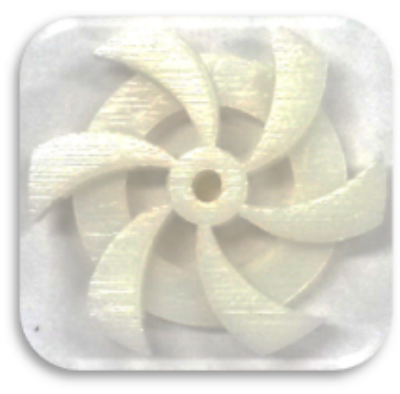

(c) Lid Inlet.

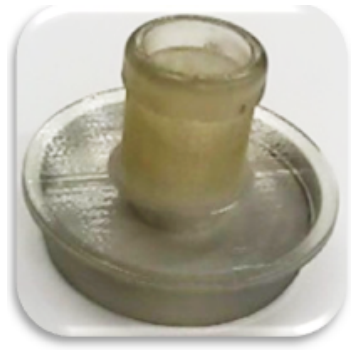

(f) Involute Blade Rotor.

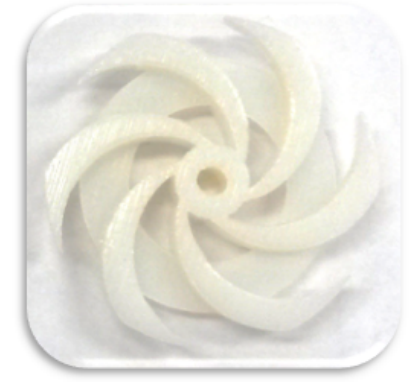

\subsubsection{Machined Parts}

The magnet ring and the silicon steel rings are built by machining. The magnet ring, as stated in Section 7.1.2 is built by assembling a PVC ring, an iron ring and the magnets. Each of these parts is made by a different process, the silicon steel rings are machined by a wire EDM (Electrical Discharge Machine) process and the PVC ring is machined on a lathe. The resulting assemble for the magnet ring is shown in Figure $7.8 \mathrm{a}$ and the external silicon steel rings are shown in Figure $7.8 \mathrm{~b}$.

Figure 7.8 - Magnet ring and silicon steel ring built.
(a) Rotor: Magnet ring assemble.
(b) Stator: External silicon steel ring.
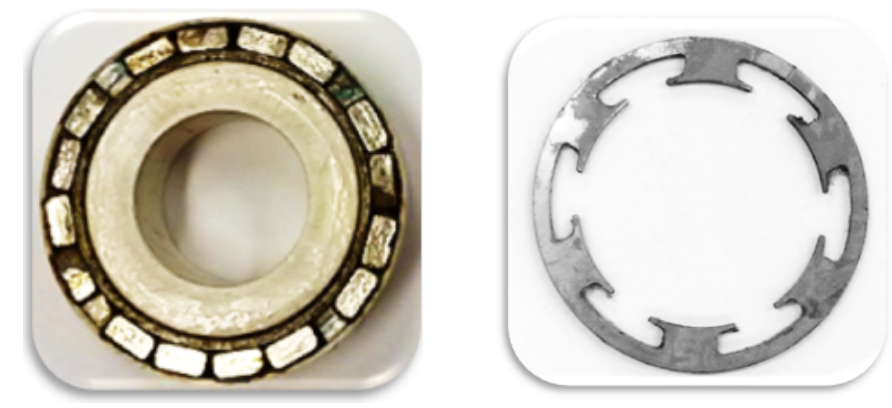


\subsubsection{Prototype Assemble}

The complete assemble of the parts shown in previous section, for both cases (with and without the volute) can be seen in Figure 7.9 .

Figure 7.9 - Prototype assemble.

(a) Prototype with volute.

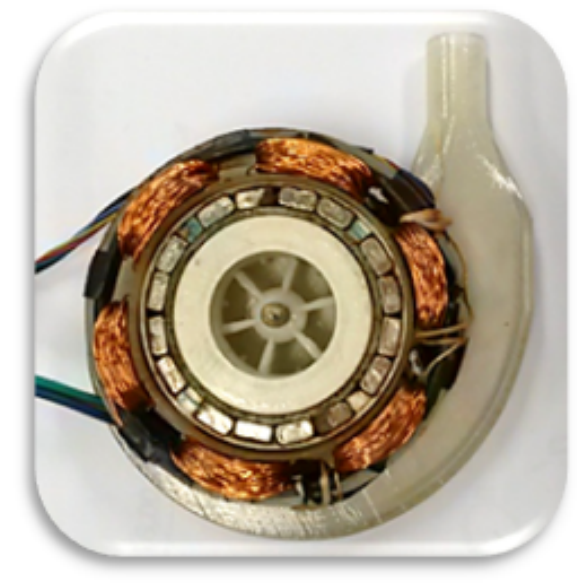

(b) Prototype without volute.

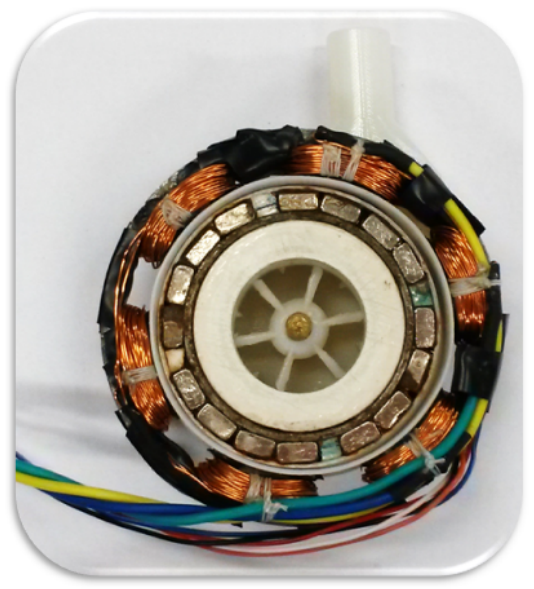

\subsection{Experimental Setup}

\subsubsection{Experimental Scheme}

In order to test the built prototype an experiment is performed. The experiment aims to measure the pressure head and the mass flow given by the pump. The experiment consist of a water tank, a flow sensor and a manometer coupled to the pump. The pump rotation speed is controlled by a commercial controller that uses a DC source. The flow sensor and the hall sensor outputs are obtained by using an oscilloscope. The scheme representing this assemble is shown in Figure 7.10 .

Figure 7.10 - Experimental setup scheme.

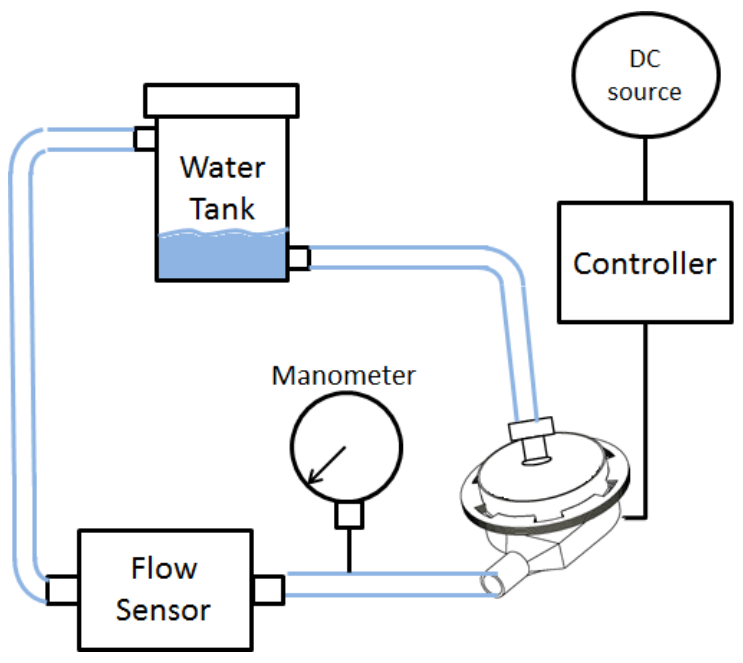




\subsubsection{Flow Sensor}

The flow sensor used is the digital sensor Flownetix 100series Smart Ultrasonic Flowmeter, which has features such as automatic viscosity and temperature compensation, low pressure drop and no moving parts. The sensor has an electric output in voltage, so, in order to measure the sensor output in relevant units, such as $l / \mathrm{min}$, an experimental calibration curve is used (Figure 7.12). The flow sensor is shown in Figure 7.11.

Figure 7.11 - Flow sensor: Flownetix 100series Smart Ultrasonic Flowmeter.

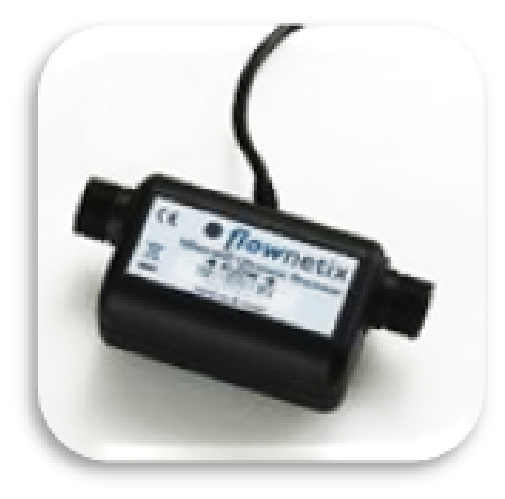

Figure 7.12 - Flow sensor experimental curve.

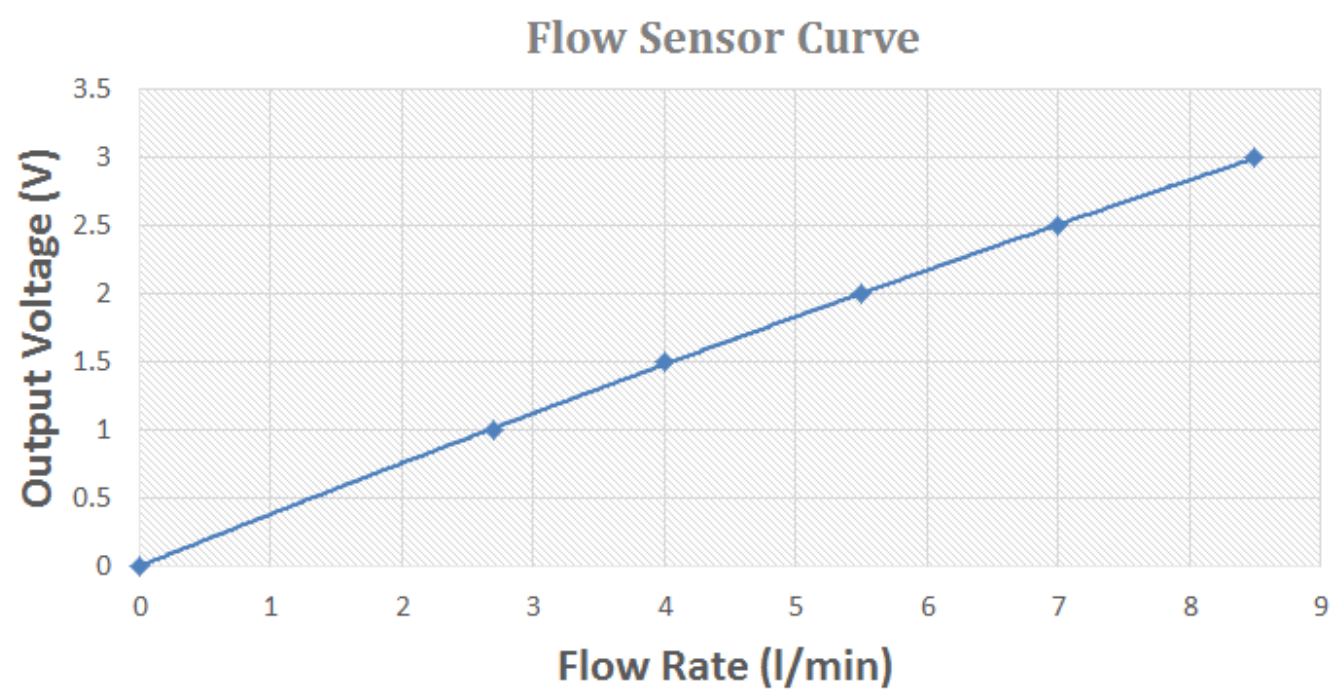

\subsubsection{Pressure Sensor}

The pressure sensor used is an analog manometer. The pressure output is given in millimeters of mercury $(\mathrm{mmHg})$ and the sensor is shown in Figure 7.13. 
Figure 7.13 - Analog manometer.

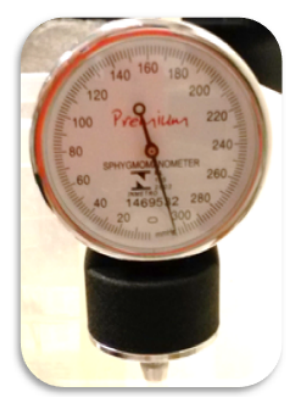

\subsection{Pump Similarity Laws}

Since pumps characteristics are usually determined experimentally, the dimensional analysis and similitude considerations are useful in the documentation of the pump characteristics. The main dependent pump variables are the head rise $\left(h_{a}\right)$, shaft power $(\dot{W})$, and efficiency $(\eta)$. These variables will depend on the geometrical configuration, which can be represented by some characteristic diameter, $D$, other pertinent lengths $l_{i}$, and surface roughness $\epsilon$. In addition, the other important variables are flowrate $(Q)$, the pump shaft rotational speed $(\omega)$, fluid viscosity $(\mu)$, and fluid density $(\rho)$.

The application of dimensional analysis gives:

$$
\text { Dependent } \pi \text { term }=\Phi\left(\frac{l_{i}}{D}, \frac{\epsilon}{D}, \frac{Q}{\omega D^{3}}, \frac{\rho \omega D^{2}}{\mu}\right)
$$

The dependent $\pi$ term involving the head $\left(h_{a}\right)$ is usually expressed as $C_{H}$, this dimensionless parameter is called the head rise coefficient. The dependent $\pi$ term involving the shaft power $\dot{W}$ is expressed as $C_{P}$ and is termed the power coefficient. The final dependent $\pi$ term is the efficiency, which is already dimensionless. These $\pi$ terms are given by (MUNSON et al., 2012):

$$
\begin{aligned}
C_{H} & =\frac{g h_{a}}{\omega^{2} D^{2}}=\Phi_{1}\left(\frac{l_{i}}{D}, \frac{\epsilon}{D}, \frac{Q}{\omega D^{3}}, \frac{\rho \omega D^{2}}{\mu}\right) \\
C_{P} & =\frac{\dot{W}}{\rho \omega^{3} D^{5}}=\Phi_{2}\left(\frac{l_{i}}{D}, \frac{\epsilon}{D}, \frac{Q}{\omega D^{3}}, \frac{\rho \omega D^{2}}{\mu}\right) \\
\eta & =\frac{\rho g Q h_{a}}{\dot{W}}=\Phi_{3}\left(\frac{l_{i}}{D}, \frac{\epsilon}{D}, \frac{Q}{\omega D^{3}}, \frac{\rho \omega D^{2}}{\mu}\right)
\end{aligned}
$$

The last $\pi$ term in each of the above equations is a form of Reynolds number that represents the relative influence of viscous effects. When the pump flow involves high Reynolds numbers, as it is usually the case, experience has shown that the effect of the Reynolds number can be neglected. For simplicity, the relative roughness $\epsilon / D$, can 
also be neglected in pumps since the highly irregular shape of the pump chamber is usually the dominant geometric factor rather than the surface roughness. Thus, with these simplifications and for geometrically similar pumps (all pertinent dimensions $l_{i}$, scaled by a common length scale), the dependent $\pi$ terms are functions of only $Q / \omega D^{3}$ so that

$$
\begin{aligned}
C_{H} & =\frac{g h_{a}}{\omega^{2} D^{2}}=\Phi_{1}\left(\frac{Q}{\omega D^{3}}\right) \\
C_{P} & =\frac{\dot{W}}{\rho \omega^{3} D^{5}}=\Phi_{2}\left(\frac{Q}{\omega D^{3}}\right) \\
\eta & =\frac{\rho g Q h_{a}}{\dot{W}}=\Phi_{3}\left(\frac{Q}{\omega D^{3}}\right)
\end{aligned}
$$

The dimensionless parameter $C_{Q}=Q / \omega D^{3}$ is called the flow coefficient. These three equations provide the desired similarity relationships among a family of geometrically similar pumps. If two pumps from the family are operated at the same value of flow coefficient

$$
\left(\frac{Q}{\omega D^{3}}\right)_{1}=\left(\frac{Q}{\omega D^{3}}\right)_{2}
$$

then follows that

$$
\begin{aligned}
\left(\frac{g h_{a}}{\omega^{2} D^{2}}\right)_{1} & =\left(\frac{g h_{a}}{\omega^{2} D^{2}}\right)_{2} \\
\left(\frac{\dot{W}}{\rho \omega^{3} D^{5}}\right)_{1} & =\left(\frac{\dot{W}}{\rho \omega^{3} D^{5}}\right)_{2} \\
\left(\frac{\rho g Q h_{a}}{\dot{W}}\right)_{1} & =\left(\frac{\rho g Q h_{a}}{\dot{W}}\right)_{2}
\end{aligned}
$$

where the subscripts 1 and 2 refer to any two pumps from the family of geometrically similar pumps.

With these pump scaling laws it is possible to experimentally determine the performance characteristics of one pump in the laboratory and then use these data to predict the corresponding characteristics for other pumps within the family and/or under different operating conditions.

In this work there is a situation in which using the scaling law is specially useful, that is predicting how the pump characteristics are affected given a change in the operating speed $\omega$. It follows from Eqs. 7.8 and 7.9 that for the same pump $\left(D_{1}=D_{2}\right)$ :

$$
\begin{aligned}
\frac{Q_{1}}{Q_{2}} & =\frac{\omega_{1}}{\omega_{2}} \\
\frac{h_{a 1}}{h_{a 2}} & =\frac{\omega_{1}^{2}}{\omega_{2}^{2}}
\end{aligned}
$$


With these relations it is necessary to measure the pressure head and mass flow for only one rotation and collect the pump characteristic curve and then use scaling laws to predict the curves for other rotations. The effect in the curve for lower and higher rotations is illustrated in Figure 7.14.

Figure 7.14 - Example of the scaling law for changes in the operating speed.

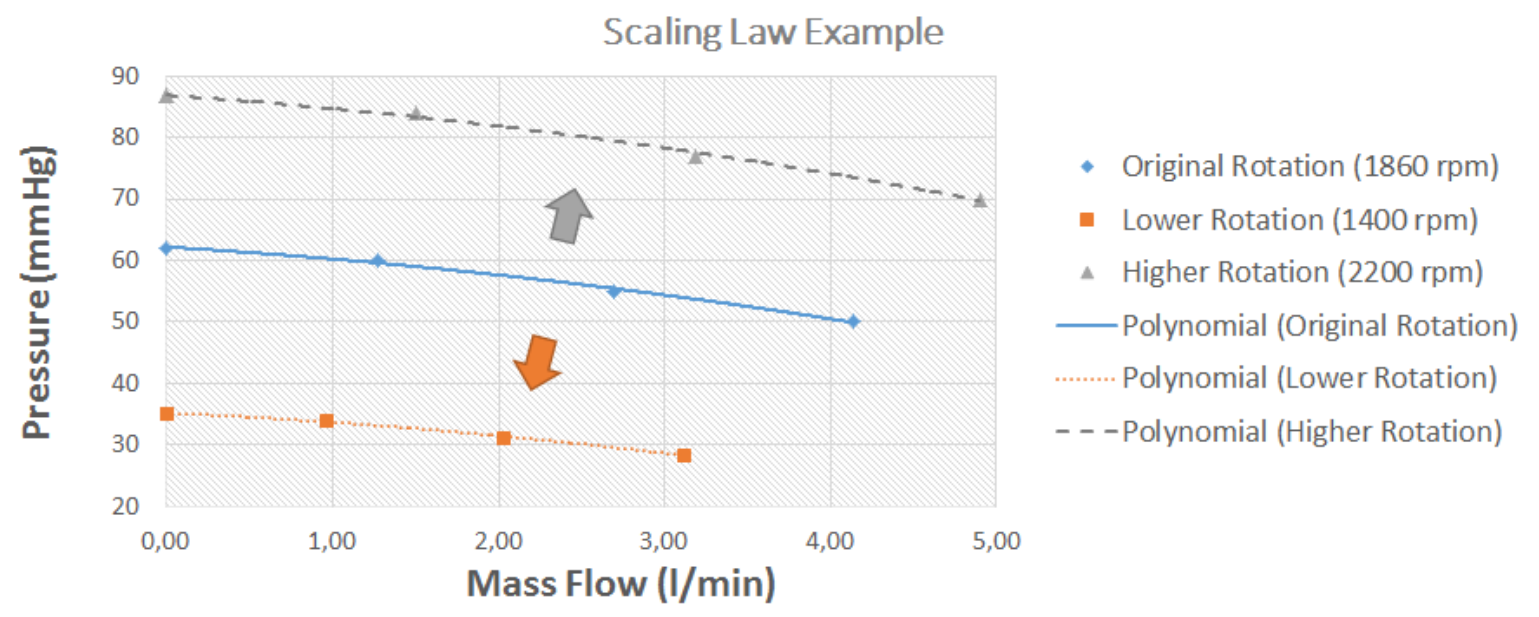




\section{EXPERIMENTAL RESULTS}

This section presents the results obtained by using the experimental site and the optimized topologies obtained. Also, results presented here are related to experiments performed by using the case with the volute (Figure 7.9a). The experimental data is collected and used as input for a computational model. This model is necessary in order to evaluate the objective function values, given that, in the experiment, only the pressure head and mass flow are measured. The section starts presenting the experimental results for three rotors and then a comparison among them is done. The three rotor are: the straight blade rotor (Fig.6.2a), the optimized for energy dissipation only (Fig.6.3a, addressed here as "Optimized $w_{e}=1^{\prime \prime}$ ) and the optimized for energy dissipation and vorticity (Fig.6.3b, addressed here as "Optimized $w_{e}=0.7 w_{v}=0.3$ ")

\subsection{Rotor Design Interpretation}

The rotors presented in this section are post-processed designs of the results shown in Section 6. Thus, a scale change is done due to the fact that the numerical results are obtained by using arbitrary dimensions (inner radius of 0.4 and outer radius of 1.0) and the prototype has smaller dimensions (inner radius of $8[\mathrm{~mm}]$ and outer radius of $21[\mathrm{~mm}]$ ), however the aspect ratio is maintained by sustaining the center of the straight blade rotor. Also, looking forward to obtain a complete rotor the resulting topologies are reproduced in a pattern in order to get a circular rotor, as shown in Figure 8.1 .

Figure 8.1 - Rotor post-processed design.

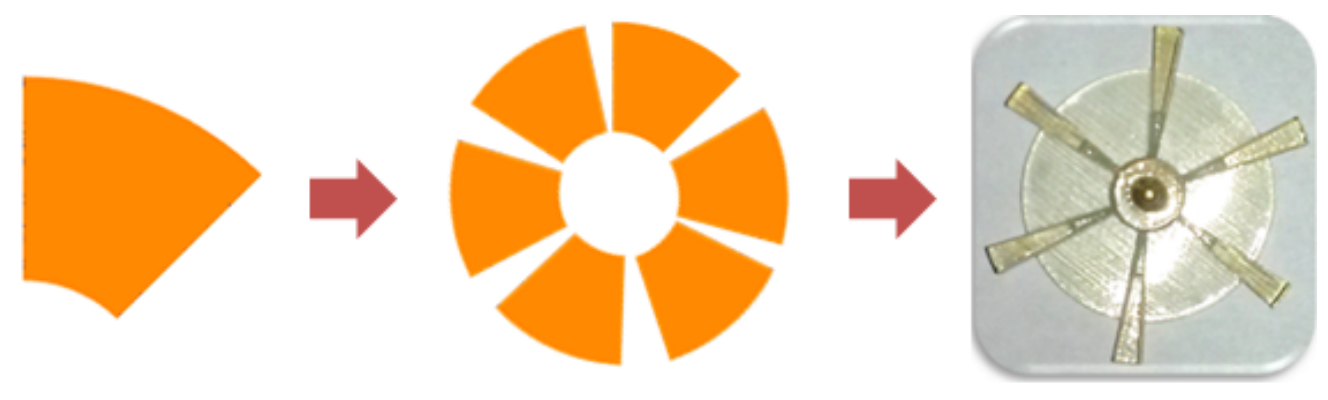

\subsection{Straight Blade Experimental Result}

The experiment measures, as said in Chapter 7, only the pump pressure head and mass flow. Thus, a curve having the mass flow in the $\mathrm{x}$ axis and the pressure in the $\mathrm{y}$ axis is built. The curve for the straight blade considering different rotations and an unrestricted flow, is shown in Figure 8.2 . The points in the curve are collected by using water and 
considering no restriction on station ducts and increasing the rotation speed. Thus, the blue curve represents the demand curve of the experimental site, i.e. it considers all the pressure loss on the channels and also the loss in the pump and is identified here as the Station curve.

The station curve has deviation bars relative to imprecision in the sensors, and then we adopted a deviation of $4[\mathrm{mmHg}]$ for the manometer and $50[\mathrm{mV}]$ for the flow sensor. The pump curve also has the same deviation. However they are not represented in this chart.

The pump curve is obtained by fixing a rotation and increasing the restriction on the station ducts by pressing them, reducing its diameter. It's important to notice that given a curve we can use the scaling laws (Eqs. 7.12 and 7.13 presented before to obtain the curves for other rotations. For example, in Figure 8.2, two curves for different rotations (1850 and $1230[\mathrm{rpm}])$ were collected.

Figure 8.2 - Experimental curves for straight blade rotor.

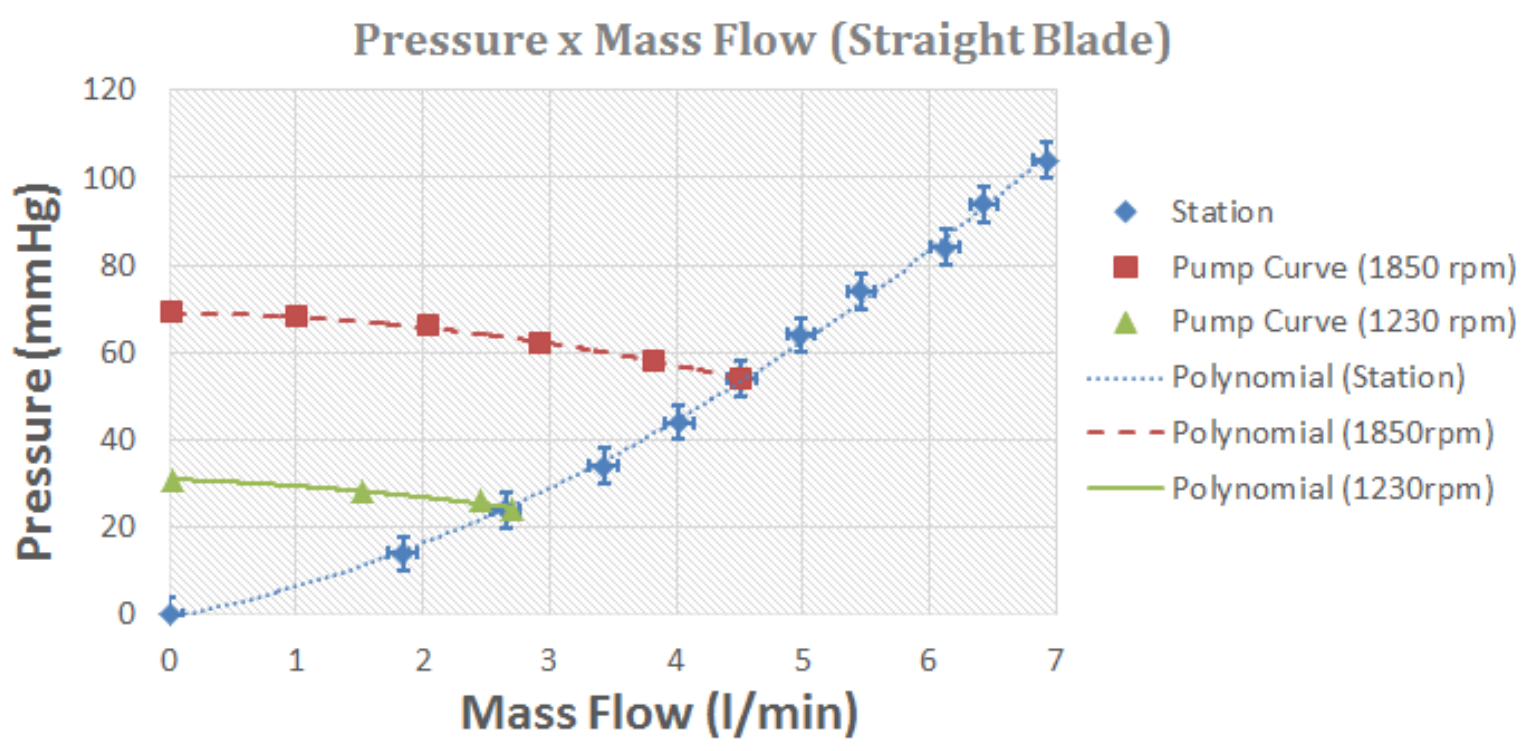

The experimental data can be used to define the boundary conditions of the computational model, by using the prototype mass flow information to define the model inlet velocity. Thus, it is possible to verify the simulation results by comparing the experimental data and the results of a complete pump model with six blades and case in the commercial software ANSYS (Fig. 8.3). The simulations are performed by using only the case with the volute, given that the model for the case without the volute do not represent well the reality, as presented in the Section 6.3. The boundary conditions are defined as: the inlet velocity (green area) is adjusted to have the mass flow of the experimental data and pressure equal to zero at the outlet, while all other faces have a no slip wall condition. Also, the ANSYS built-in k- $\epsilon$ turbulence model is used to get better results at the verification. 
The number of elements in the mesh is defined by performing a mesh convergence test. The test verify the change in a control value, in this case the pressure at the inlet, with respect to the number of elements in the mesh. Thus, the curve of Table 8.1 shows the mesh convergence for the mass flow of $4.5[\mathrm{l} / \mathrm{min}]$ and rotation of $1720[\mathrm{rpm}]$.

Table 8.1 - Mesh convergence of ANSYS model.

\begin{tabular}{ccccccc}
\hline $\begin{array}{c}\text { Number of } \\
\text { elements }\end{array}$ & $\begin{array}{c}\text { Pressure } \\
\text { (Inlet) }\end{array}$ & \% Change & $\begin{array}{c}\text { Energy } \\
\text { Dissipation }\end{array}$ & \% Change & Vorticity & \% Change \\
\hline 20,254 & $-7,729$ & - & 0.25 & - & 199.75 & - \\
162,452 & $-7,878$ & $1.9 \%$ & 0.54 & $119.3 \%$ & 503.19 & $151.9 \%$ \\
328,163 & $-7,741$ & $-1.7 \%$ & 0.59 & $8.8 \%$ & 544.16 & $8.1 \%$ \\
496,959 & $-7,348$ & $-5.1 \%$ & 0.58 & $-1.5 \%$ & 555.65 & $2.1 \%$ \\
768,820 & $-7,479$ & $1.8 \%$ & 0.60 & $3.3 \%$ & 575.00 & $3.5 \%$ \\
\hline
\end{tabular}

The simulation results are illustrated in Figure 8.4, which shows the absolute velocity and pressure fields for the mass flow of $4.5[\mathrm{l} / \mathrm{min}]$, a mesh with 770,000 elements and using water as fluid. Repeating the process for some experimental points we can build the computational pressure versus mass flow curve, as shown in Figure 8.5. We can see that both curves have a good agreement in general.

The functional values for the computational model are shown in Table 8.2, these values are calculated by using a horizontal cutting plane at the middle of the pump to incorporate the effects of the rotor and volute.

It is important to notice that the ANSYS model built can only represent the unrestricted flow situations, thus, only the station curve can be reproduced and compared. In order to simulate the pump curve, that has a fixed rotation and variable mass flows, it is necessary to create a more complex model that considers the pressure drop over the ducts, which is out of the scope of the present work but can be explored in future work.

Figure 8.3 - Complete pump ANSYS model for the straight blade.

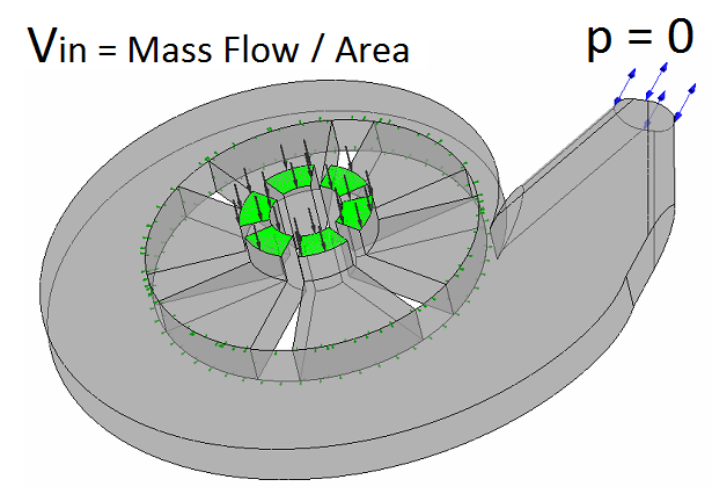


Figure 8.4 - ANSYS result for straight blade rotor at $1815[\mathrm{rpm}]$ and $4.5[\mathrm{l} / \mathrm{min}]$.

(a) Absolute Velocity Field.

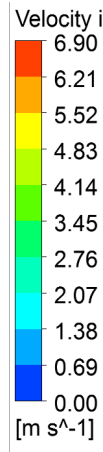

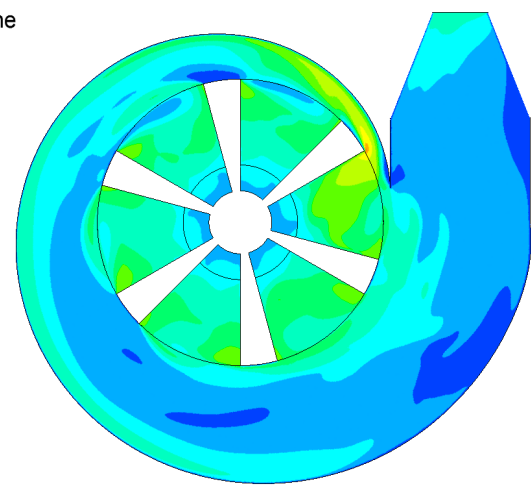

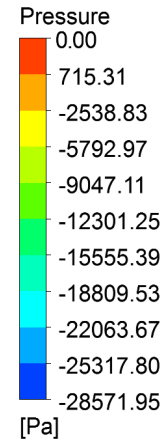

(b) Pressure Field.

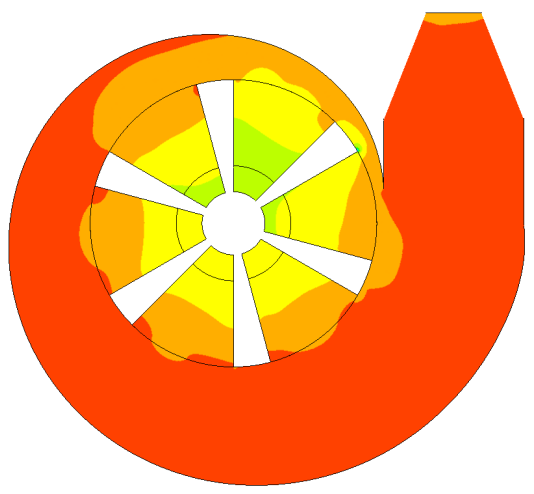

Figure 8.5 - Results comparison for Straight blade.

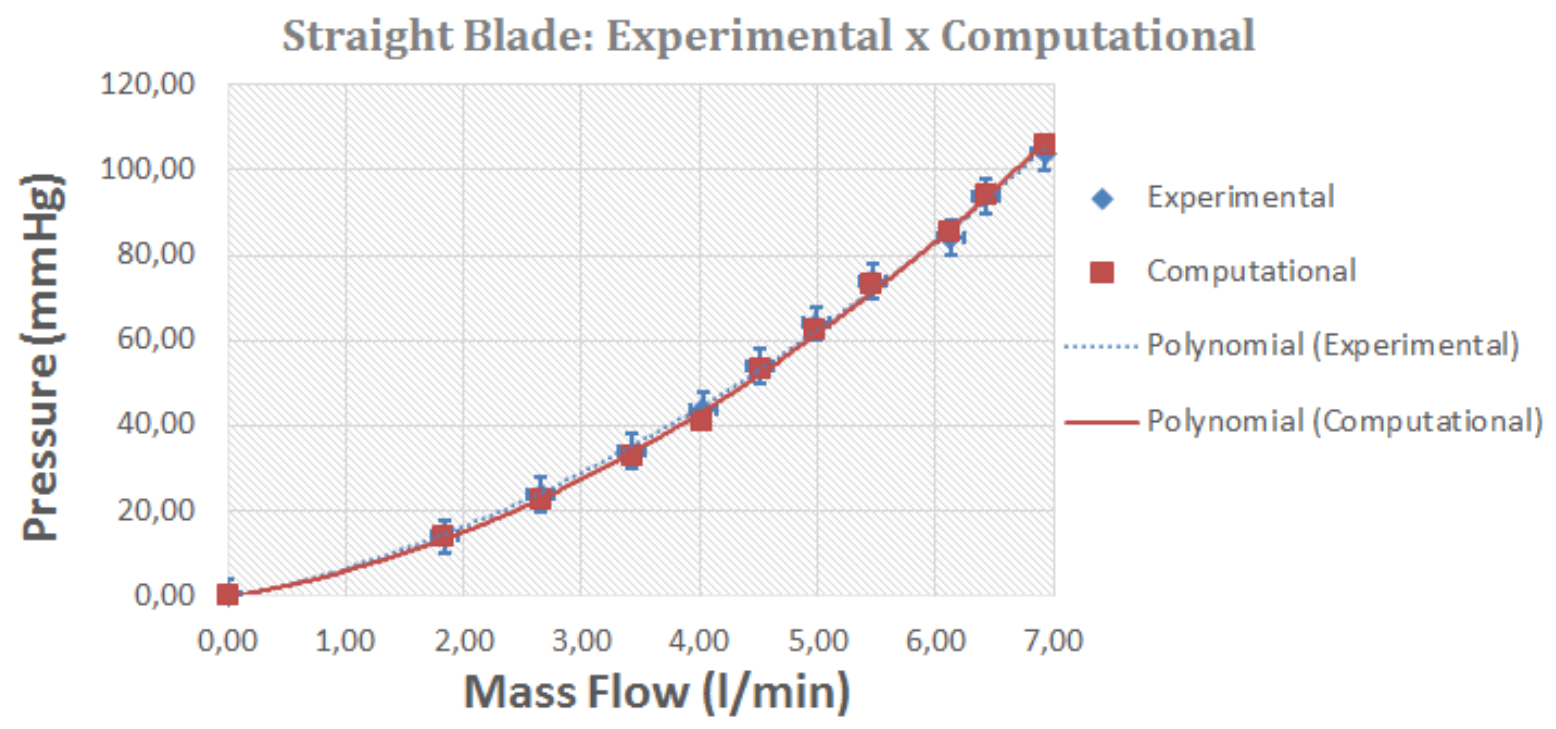

Table 8.2 - Functional values results for straight blade ANSYS model..

\begin{tabular}{ccccc}
\hline $\begin{array}{c}\text { Rotation } \\
{[\mathrm{rpm}]}\end{array}$ & $\begin{array}{c}\text { Mass Flow } \\
{[\mathrm{l} / \mathrm{min}]}\end{array}$ & $\begin{array}{c}\text { Pressure } \\
{[\mathrm{mmHg}]}\end{array}$ & $\begin{array}{c}\text { Energy } \\
\text { Dissipation }\end{array}$ & Vorticity \\
\hline 920 & 1,84 & 13,76 & 0,06 & 63,13 \\
1.208 & 2,65 & 22,74 & 0,11 & 120,40 \\
1.485 & 3,43 & 32,83 & 0,15 & 166,14 \\
1.655 & 4,03 & 41,17 & 0,22 & 233,31 \\
1.815 & 4,52 & 53,00 & 0,28 & 299,48 \\
2.010 & 4,99 & 62,61 & 0,34 & 370,22 \\
2.190 & 5,46 & 73,39 & 0,41 & 428,36 \\
2.340 & 6,13 & 85,32 & 0,52 & 554,09 \\
2.498 & 6,43 & 93,92 & 0,58 & 607,57 \\
2.643 & 6,93 & 105,67 & 0,66 & 693,83 \\
\hline
\end{tabular}




\subsection{Optimized Rotor for Energy Dissipation}

Considering the optimized blade obtained by using a straight blade as initial guess and only the energy dissipation functional (Figure 6.3) and the interpretation process cited above (Figure 8.1) the rotor shown in Figure 8.6 is built. The curve pressure versus mass flow for this rotor considering the same conditions of the previous section, i.e., increasing rotations and an unrestricted flow, is shown in Figure 8.7. Again in this figure two curves for the pump with two rotation are shown and the curve for any other rotation can be calculated using the similarity laws.

Figure 8.6 - Rotors built from topology optimization results considering straight blade as initial guess and energy dissipation functional shown in Fig. 6.3a.

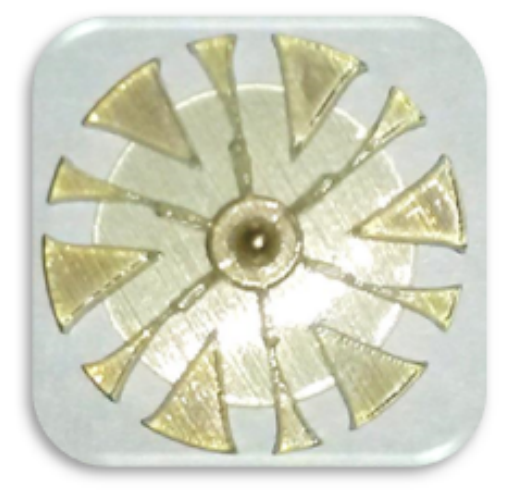

Figure 8.7 - Pump experimental curve for rotor of Fig. 8.6

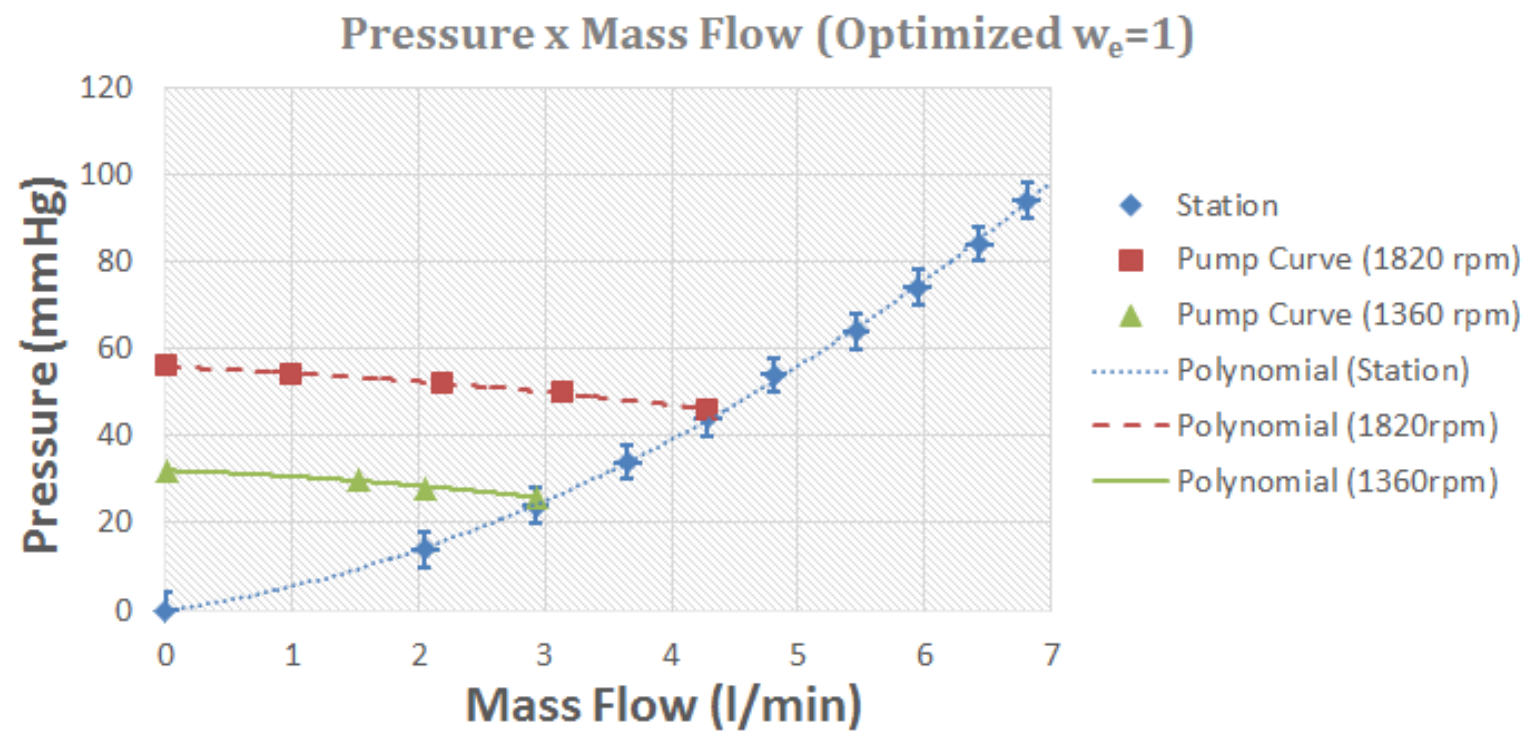

Following the same procedure presented in sections above, the boundary conditions of the computational model are defined. The boundary conditions are defined as: the inlet velocity (green area) is adjusted to have the mass flow of the experimental data and pressure equal to zero at the outlet, while all other faces have a no slip wall condition. 
Also, to define the mesh refinement a similar mesh convergence process, shown in the previous section, was performed.

The complete pump model with six blades is built, as shown in Figure 8.8, and the absolute velocity and pressure fields for the mass flow of $4.29[\mathrm{l} / \mathrm{min}]$, rotation of $1833[\mathrm{rpm}]$ and a mesh with 1 million elements are shown in Figure 8.9. The complete curve for some experimental points and the comparison with the original points are shown in Figure 8.10 Again, we can see that both experimental and computational curves are close to overlap. The difference in the curves is related to the fact that the model do not comprise all losses in the pump station, hence, only the rotor dissipation is considered.

Table 8.3 shows the rotations and mass flows used to build the comparison curve and also the objective function values for energy dissipation and vorticity, given that power is a function of pressure and mass flow. These function values are calculated only in the rotor.

Figure 8.8 - Complete pump ANSYS model of optimized rotor for energy dissipation and straight blade as initial guess (Fig. 6.3a).

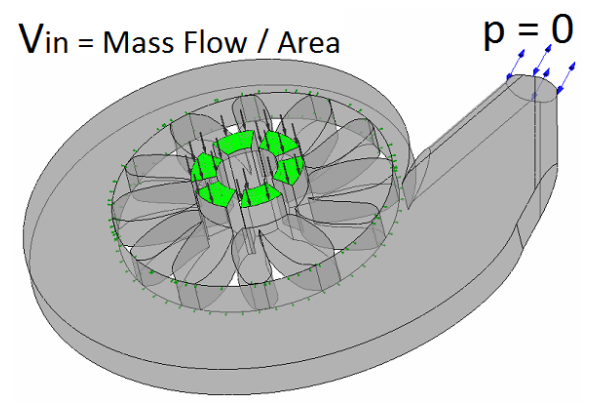

Figure 8.9 - ANSYS result for optimized rotor obtained considering energy dissipation only (Fig. 6.3a), 1833[rpm] and 4.29[l/min $]$.

(a) Absolute Velocity Field.

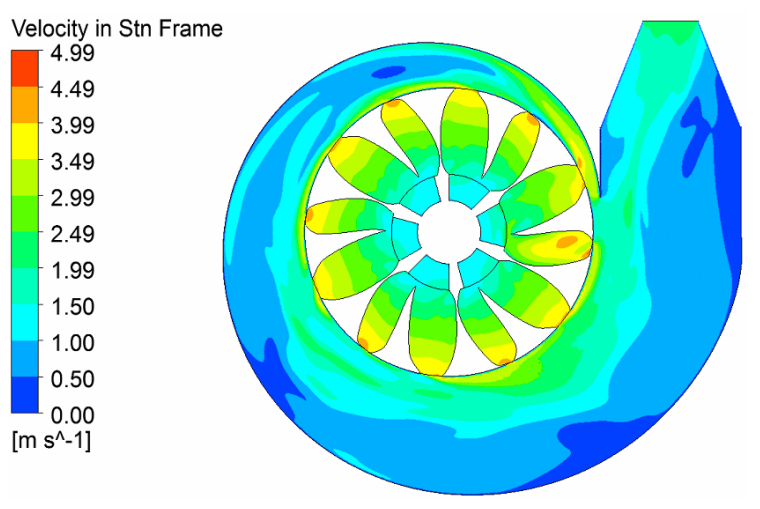

(b) Pressure Field.

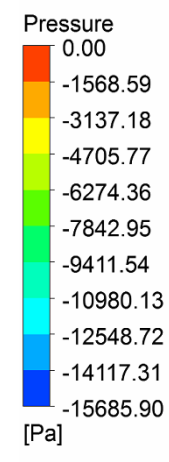

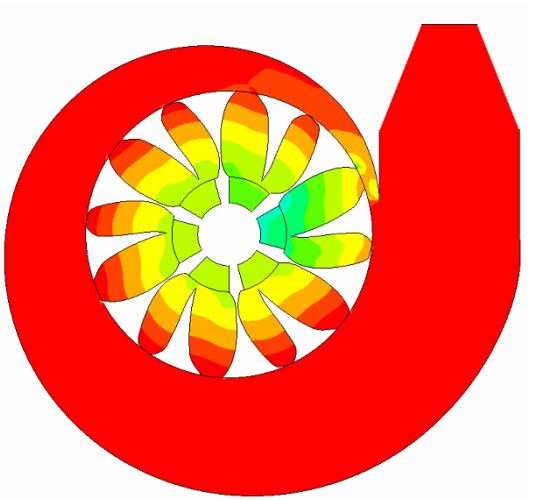


Figure 8.10 - Comparison between experimental and computational results for Optimized blade obtained by considering energy dissipation only.

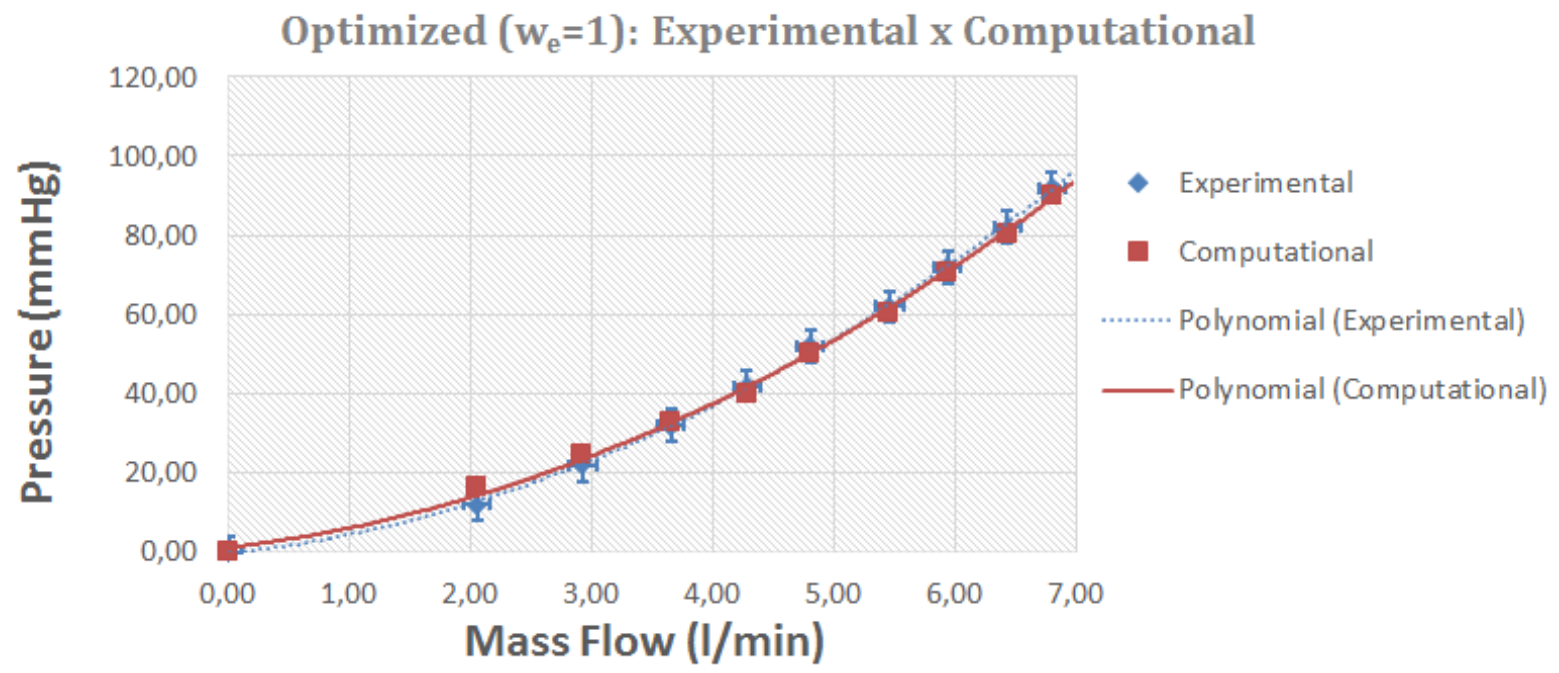

Table 8.3 - Functional values results for the ANSYS model of optimized rotor considering straight blade as initial guess and energy dissipation only as objective function.

\begin{tabular}{ccccc}
\hline $\begin{array}{c}\text { Rotation } \\
{[\mathrm{rpm}]}\end{array}$ & $\begin{array}{c}\text { Mass Flow } \\
{[\mathrm{l} / \mathrm{min}]}\end{array}$ & $\begin{array}{c}\text { Pressure } \\
{[\mathrm{mmHg}]}\end{array}$ & $\begin{array}{c}\text { Energy } \\
\text { Dissipation }\end{array}$ & Vorticity \\
\hline 1.091 & 2,05 & 16,22 & 0,03 & 26,10 \\
1.386 & 2,93 & 24,85 & 0,05 & 49,83 \\
1.631 & 3,66 & 32,88 & 0,09 & 79,54 \\
1.833 & 4,29 & 40,20 & 0,12 & 110,89 \\
1.923 & 4,81 & 50,27 & 0,15 & 137,65 \\
2.132 & 5,46 & 60,45 & 0,19 & 177,81 \\
2.279 & 5,94 & 70,40 & 0,23 & 209,31 \\
2.433 & 6,43 & 80,26 & 0,27 & 240,64 \\
2.585 & 6,80 & 90,09 & 0,32 & 278,74 \\
2.718 & 7,21 & 100,06 & 0,36 & 320,29 \\
\hline
\end{tabular}

\subsection{Optimized Rotor for Energy Dissipation and Vorticity}

Considering, now, the optimized result shown in Figure 6.3 and the interpretation process cited before (Figure 8.1) the rotor for the energy dissipation and vorticity minimization is obtained. However, the post-processing in this case is more complex, given that the numerical blade result presents a porous region with intermediary values for the design variable, therefore, the final blade contour is obtained by using an average value for the inverse permeability to define the final geometry. This process changes the optimized topology and also the velocity and pressure distributions, which affects the fluid flow and the functional values as a consequence. 
The built rotor is shown in Figure 8.11. The curve pressure versus mass flow for this case considering different rotations and unrestricted flow (Station curve) and two pump curves for 1810 and $1220[\mathrm{rpm}]$ are shown in Figure 8.12 .

Figure 8.11 - Rotors built from topology optimization results considering straight blade as initial guess and energy dissipation and vorticity functionals (Fig.6.3b).

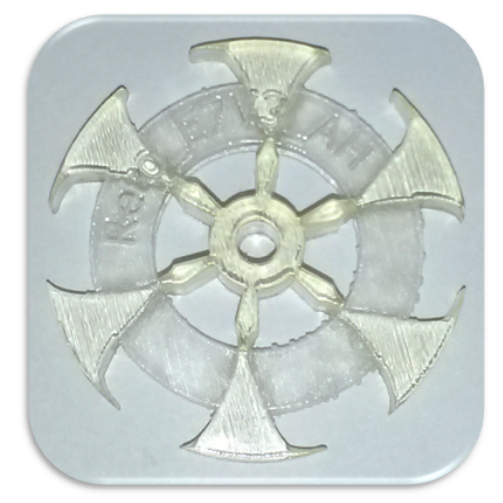

Figure 8.12 - Pump experimental curve for rotor of Fig, 8.11

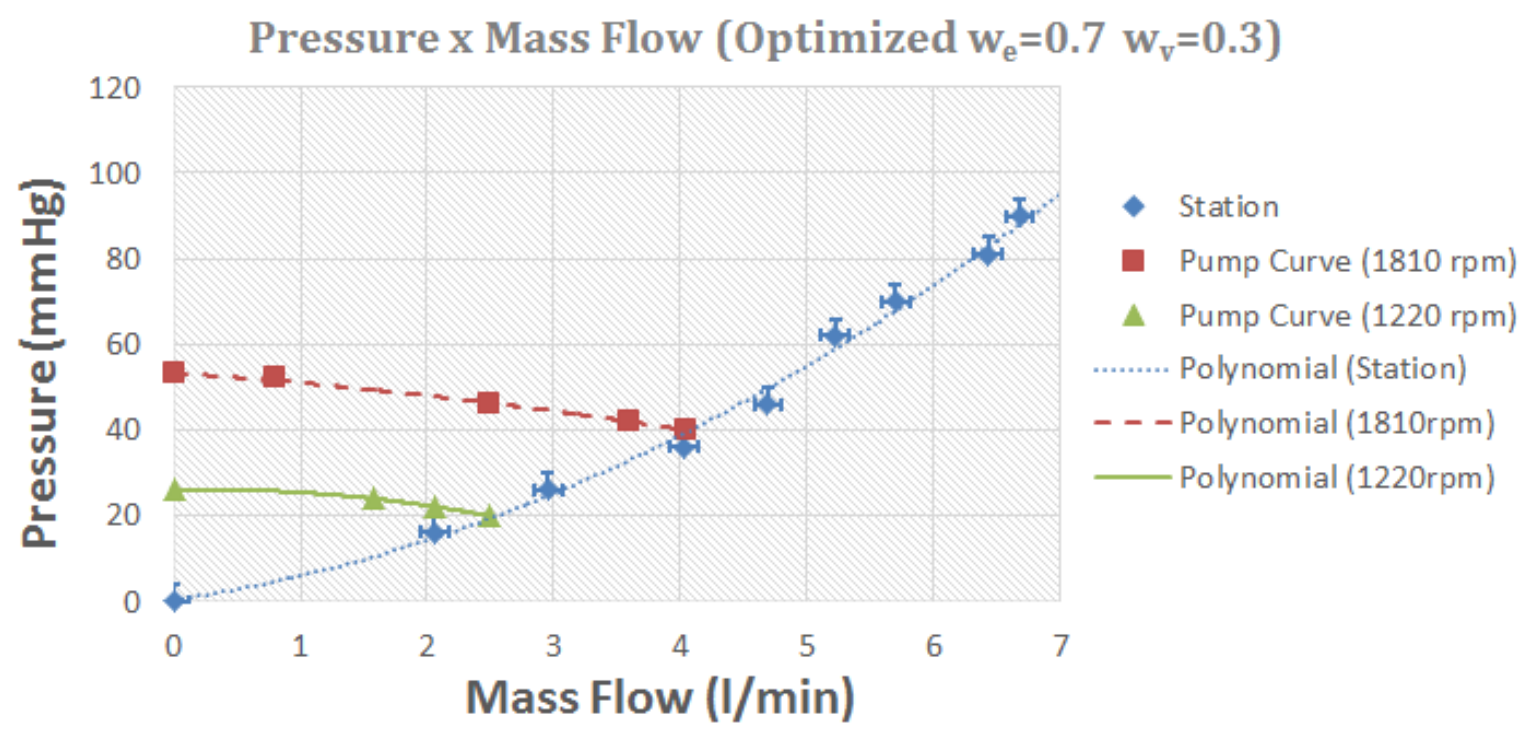

Following the same process shown in sections above, the boundary conditions of the computational model are defined by using the experimental data. The complete pump model, including rotor and volute, is shown in Figure 8.13, and the absolute velocity and pressure fields for the mass flow of $4.69[\mathrm{l} / \mathrm{min}]$ and a mesh with 800,000 elements are shown in Figure 8.14. The complete curve for some experimental points and the comparison with the computational points are shown in Figure 8.15. Again, we can see that both curves have a good agreement. Table 8.4 shows the values for energy dissipation and vorticity for different rotations and mass flows. 
Figure 8.13 - Complete pump ANSYS model of optimized rotor for energy dissipation and vorticity (Fig. 8.11).

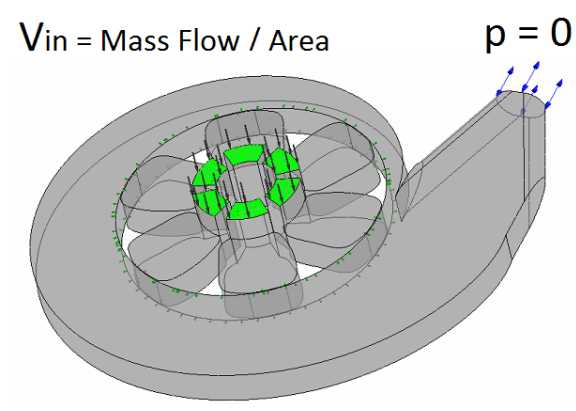

Figure 8.14 - ANSYS result for optimized rotor of Fig. 8.11. 2006 [rpm $]$ and 4.69[l/min $]$.

(a) Absolute Velocity Field.

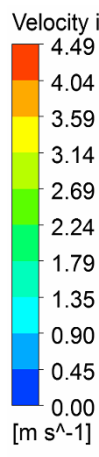

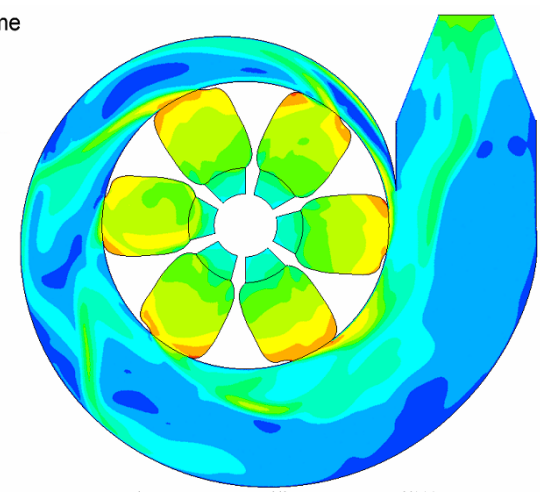

(b) Pressure Field.
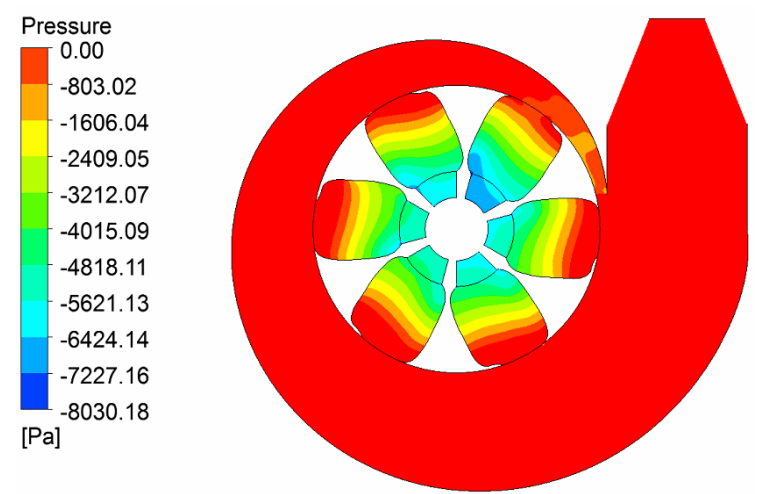

Figure 8.15 - Comparison between experimental and computational results for Optimized blade obtained by considering energy dissipation and vorticity.

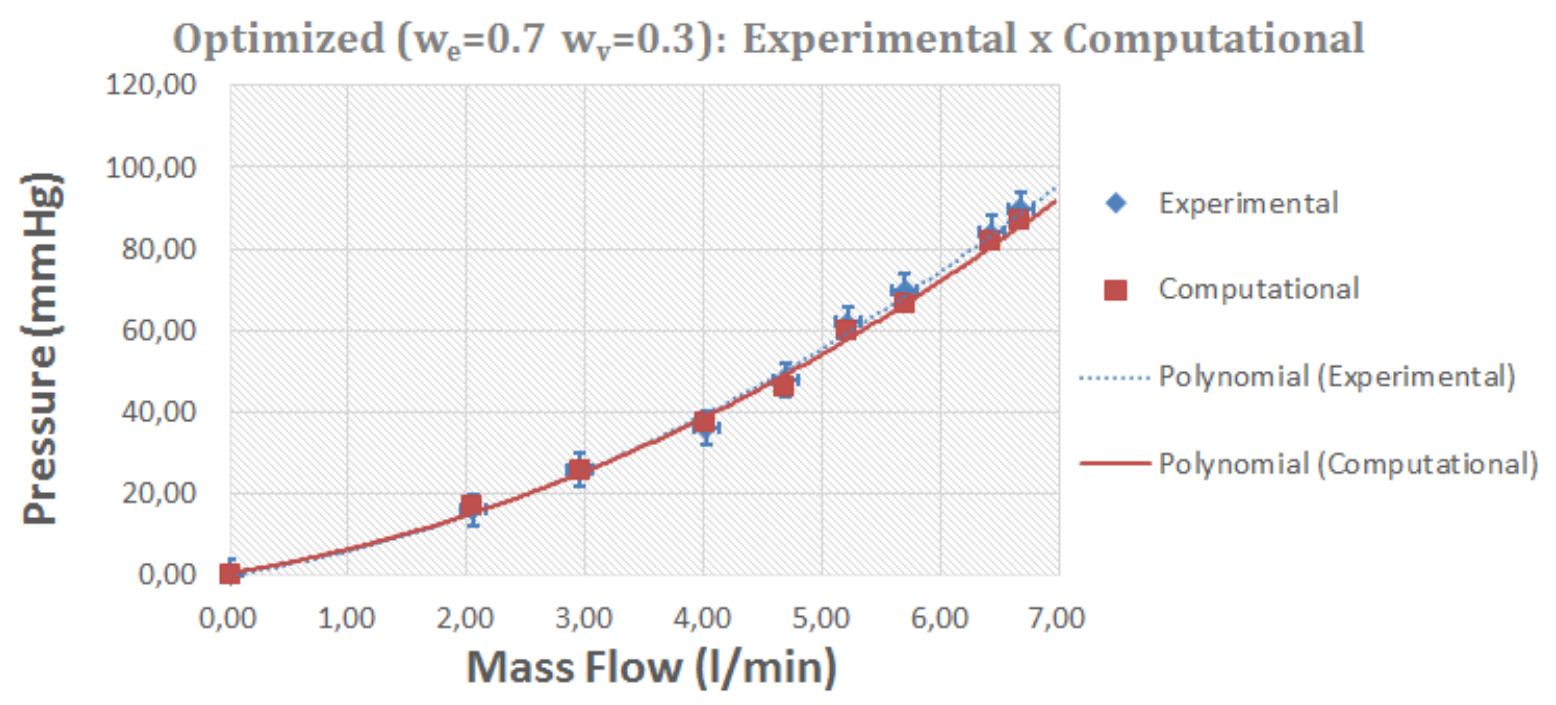


Table 8.4 - Functional values results for the ANSYS model of optimized rotor considering straight blade as initial guess and energy dissipation and vorticity.

\begin{tabular}{ccccc}
\hline $\begin{array}{c}\text { Rotation } \\
{[\mathrm{rpm}]}\end{array}$ & $\begin{array}{c}\text { Mass Flow } \\
{[\mathrm{l} / \mathrm{min}]}\end{array}$ & $\begin{array}{c}\text { Pressure } \\
{[\mathrm{mmHg}]}\end{array}$ & $\begin{array}{c}\text { Energy } \\
\text { Dissipation }\end{array}$ & Vorticity \\
\hline 1125 & 2,05 & 16,99 & 0,05 & 54,49 \\
1427 & 2,95 & 25,62 & 0,10 & 110,63 \\
1779 & 4,03 & 37,17 & 0,18 & 186,84 \\
2006 & 4,69 & 46,06 & 0,23 & 241,02 \\
2222 & 5,22 & 59,84 & 0,31 & 325,69 \\
2372 & 5,70 & 66,77 & 0,37 & 391,27 \\
2574 & 6,43 & 81,77 & 0,46 & 485,10 \\
2733 & 6,68 & 86,81 & 0,54 & 568,19 \\
2898 & 7,18 & 94,60 & 0,51 & 529,81 \\
\hline
\end{tabular}

\subsection{Experimental Results Analysis}

This section presents the comparisons among the three rotors by using the simulation data. Firstly, the pressure comparison is done to verify the simulations, then the objective functions are compared. Also, to illustrate this approach for a blood pump the comparison of wall shear stress for all the cases is presented in Appendix B.

\subsubsection{Pressure comparison}

With the results obtained from the computational model, some conclusions can be stated. The first comparison is made in term of pressure and mass flow, as shown in Figure 8.16. In this chart we observe that the three rotors have approximately same pressure output for each mass flow value, however with few deviations for high flow rates. This is due to the fact that the points listed in the chart are obtained for the free-flow condition, i.e., without flow restrictions on the duct and then they represent the pressure demand curve of the system. Thus, the higher the system resistance higher the pressure jump. Hence, the differences can be related to the energy loss inside the rotor and to the interaction between rotor and volute, indicating that the optimized rotors have lower values of viscous energy dissipation. 
Figure 8.16 - Comparison of experimental pressure measured for the three rotors.

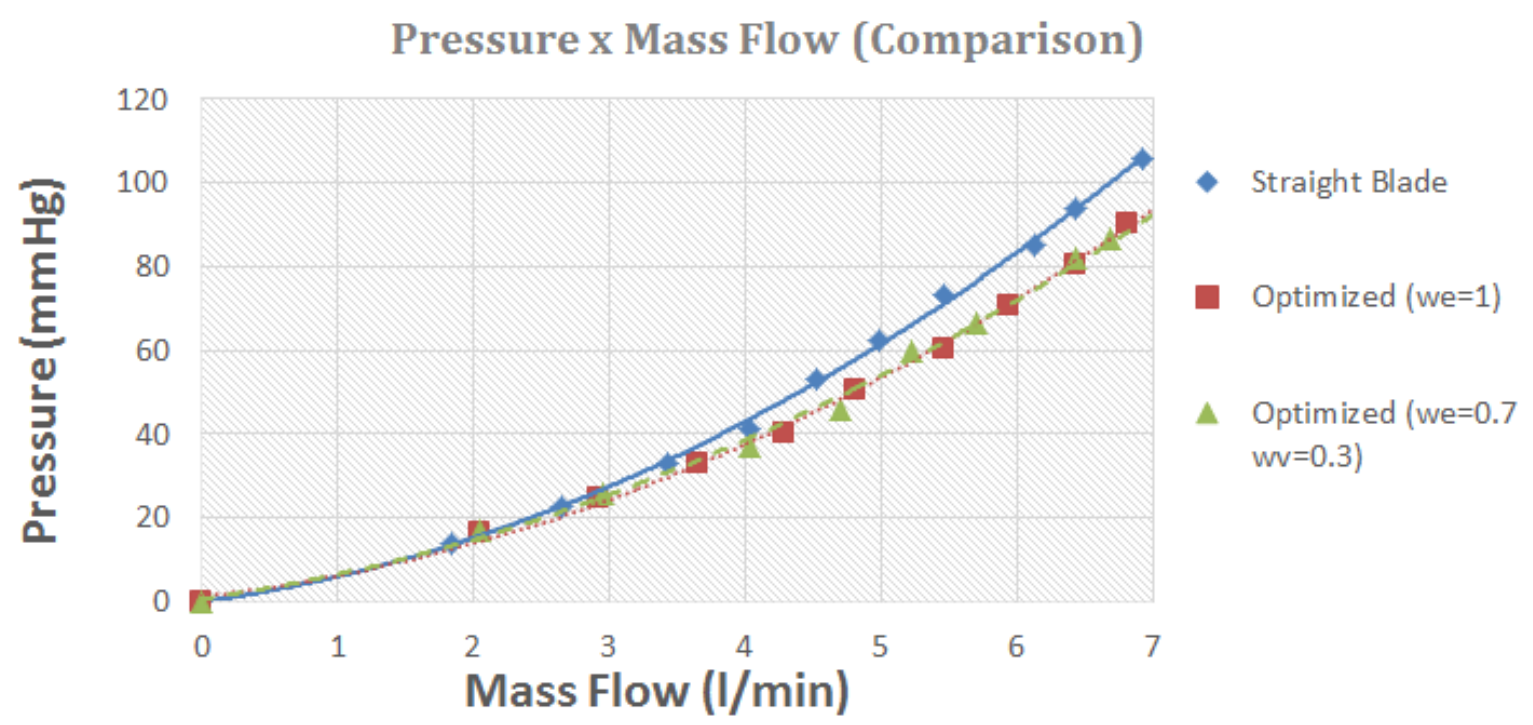

\subsubsection{Energy dissipation comparison}

Analysing the charts presented in Figures 8.5, 8.10 and 8.15 it is possible to infer that the models are coherent with the experimental results for mass flows lower than $5[l / \mathrm{min}]$. Hence, given that the computational model is verified, the simulations done in the model will reproduce the behavior of the prototype. Next, the second comparison is done by compiling the energy dissipation information of Tables 8.2, 8.3 and 8.4 and building the chart shown in Figure 8.17. These functional values are calculated by using a horizontal cutting plane at the middle of the pump and calculating the functional value given by Eq. 3.4. The middle plane is used to incorporate the effects of the rotor and volute.

Figure 8.17 shows that both optimized rotors have a lower energy dissipation value for all points collected. Using the interpolation equations for each curve (Eq 8.1) and establishing a series of fixed values of mass flow it is possible to compare the energy dissipation values in an equal scale.

$$
\begin{aligned}
& y=0,0141 x^{2}-0,0027 x+0,0061 \quad \text { Straight Blade } \\
& y=0,0074 x^{2}-0,0048 x+0,0024 \quad \text { Optimized }\left(w_{e}=1.0\right) \\
& y=0,0099 x^{2}+0,0072 x-0,0045 \quad \text { Optimized }\left(w_{e}=0.7, w_{v}=0.3\right)
\end{aligned}
$$


Figure 8.17 - Comparison of computational energy dissipation for the three rotors.

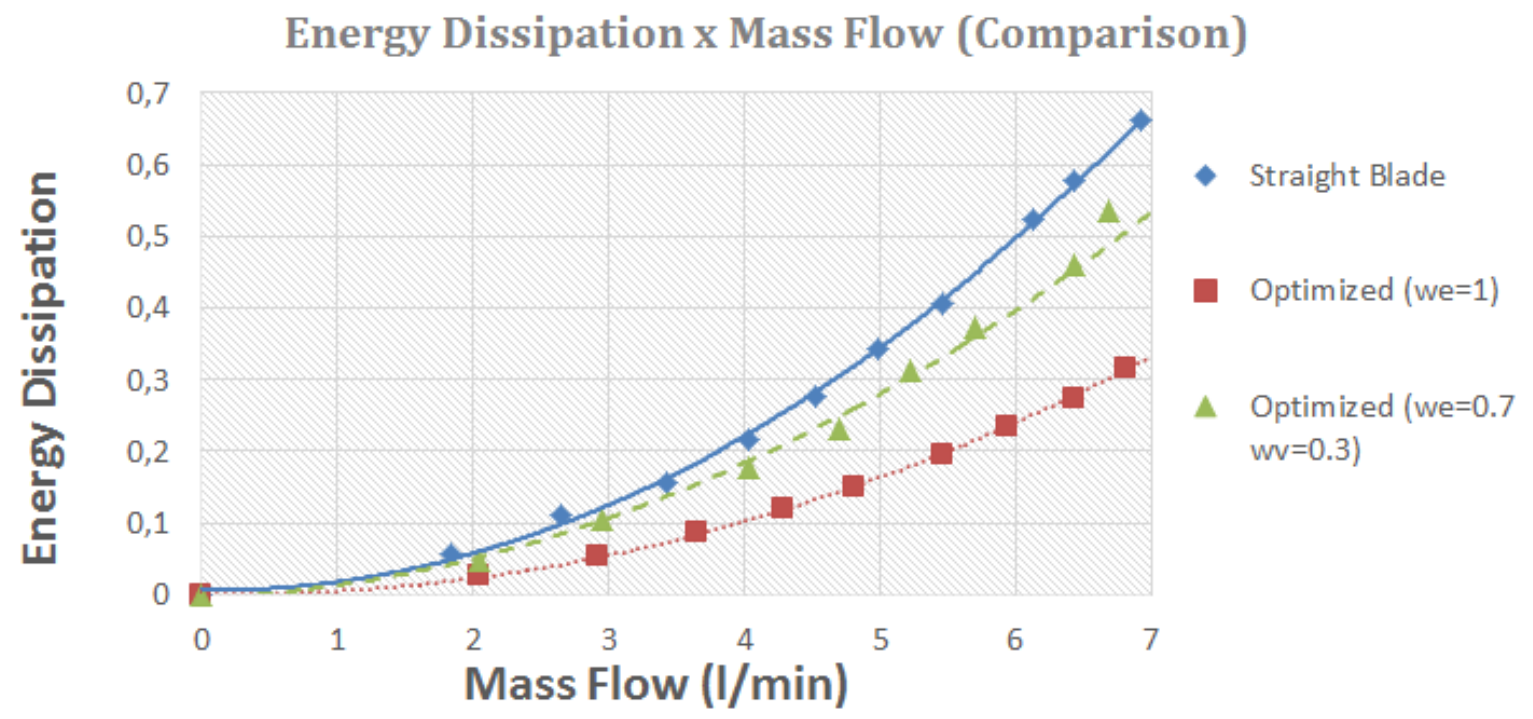

Table 8.5 shows the interpolated values for the three rotors, and, also, a column with the percentage change from the original rotor. It is possible to notice that the optimized rotor for energy dissipation only (Figure 6.3a) has an average decrease of $57 \%$ for the energy dissipation function, while the rotor optimized for energy dissipation and vorticity has an average decrease of $19 \%$ when compared with the straight blade rotor.

Table 8.5 - Interpolated values for energy dissipation.

\begin{tabular}{|c|c|c|c|c|c|}
\hline \multicolumn{6}{|c|}{ Energy Dissipation } \\
\hline Mass Flow & Straight Blade & \multicolumn{2}{|c|}{ Optimized $\left(w_{e}=1\right)$} & \multicolumn{2}{|c|}{ Optimized $\left(w_{e}=0.7, w_{v}=0.3\right)$} \\
\hline$[l / \min ]$ & Value & Value & $\%$ change & Value & $\%$ change \\
\hline 1 & 0,02 & 0,00 & $-72 \%$ & 0,01 & $-27 \%$ \\
\hline 2 & 0,06 & 0,02 & $-61 \%$ & 0,05 & $-13 \%$ \\
\hline 3 & 0,12 & 0,05 & $-56 \%$ & 0,11 & $-15 \%$ \\
\hline 4 & 0,22 & 0,10 & $-54 \%$ & 0,18 & $-17 \%$ \\
\hline 5 & 0,35 & 0,16 & $-53 \%$ & 0,28 & $-19 \%$ \\
\hline 6 & 0.50 & 0,24 & $-52 \%$ & 0,40 & $-20 \%$ \\
\hline 7 & 0,68 & 0,33 & $-51 \%$ & 0.53 & $-21 \%$ \\
\hline Average & & & $-57 \%$ & & $-19 \%$ \\
\hline
\end{tabular}

\subsubsection{Vorticity comparison}

The third comparison is done by compiling the vorticity information of Tables 8.2 , 8.3 and 8.4 and building the chart shown in Figure 8.18. These functional values are calculated by using the middle plane, as said in the previous section, and by calculating the functional value given by Eq. 3.7 . 
Figure 8.18 - Comparison of computational vorticity for the three rotors.

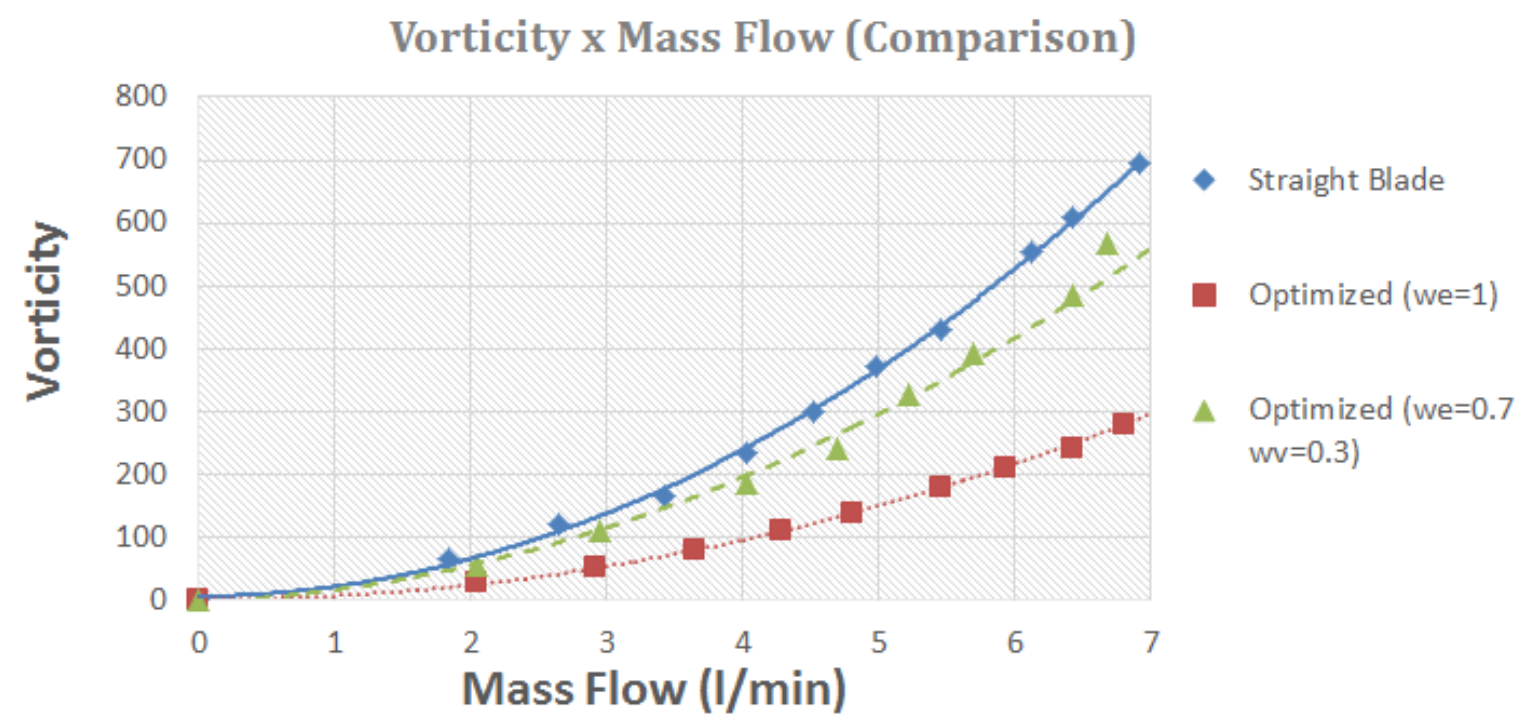

This chart (Figure 8.18), again, shows that both optimized rotors have better value for the objective function, in this case vorticity. In order to compare the changes in the functional the interpolation equations for each curve are used $(\mathrm{Eq} .8 .2$ ) and the vorticity values are compared in an equal basis.

$$
\begin{aligned}
& y=14,2166 x^{2}+1,4825 x+4,5363 \quad \text { Straight Blade } \\
& y=6,2368 x^{2}-1,6866 x+1,3697 \quad \text { Optimized }\left(w_{e}=1.0\right) \\
& y=10,2312 x^{2}+8,7767 x-4,4047 \quad \text { Optimized }\left(w_{e}=0.7, w_{v}=0.3\right)
\end{aligned}
$$

Table 8.6 shows the interpolated values for the three rotors, and, also, a column with the percentage change from the original rotor. It is possible to notice that the optimized rotor for energy dissipation $\left(w_{e}=1.0\right.$ - Figure 6.3a) has an average decrease of $62 \%$ for the vorticity function, while the rotor optimized for energy dissipation and vorticity $\left(w_{e}=0.7, w_{v}=0.3\right.$ - Figure 6.3b has an average decrease of $20 \%$ when compared with the straight blade rotor. The small improvement in the second case can be related to the post-processing procedure, where the green region (representing an intermediary porosity) is removed and replaced with an interpolated spline, which changes the topology and also the flow behavior, thus, influencing the blade characteristics. 
Table 8.6 - Interpolated values for vorticity.

\begin{tabular}{c|c|cc|cc}
\hline \multicolumn{7}{c}{ Vorticity } \\
\hline $\begin{array}{c}\text { Mass Flow } \\
{[l / \text { min }]}\end{array}$ & $\begin{array}{c}\text { Straight Blade } \\
\text { Value }\end{array}$ & \multicolumn{2}{c}{ Optimized $\left(w_{e}=1\right)$} & \multicolumn{2}{l}{ Optimized $\left(w_{e}=0.7, w_{v}=0.3\right)$} \\
Value & $\%$ change & Value & \% change \\
\hline 1 & 20,24 & 5,92 & $-71 \%$ & 14,60 & $-28 \%$ \\
2 & 64,37 & 22,94 & $-64 \%$ & 54,07 & $-16 \%$ \\
3 & 136,93 & 52,44 & $-62 \%$ & 114,01 & $-17 \%$ \\
4 & 237,93 & 94,41 & $-60 \%$ & 194,40 & $-18 \%$ \\
5 & 367,36 & 148,86 & $-59 \%$ & 295,26 & $-20 \%$ \\
6 & 525,23 & 215,78 & $-59 \%$ & 416,58 & $-21 \%$ \\
7 & 711,53 & 295,17 & $-59 \%$ & 558,36 & $-22 \%$ \\
\hline Average & \multicolumn{5}{c}{$-62 \%$} \\
\hline
\end{tabular}

\subsubsection{Power comparison}

The last comparison is done indirectly by using the relations Power $=$ Pressure . MassFlow. However, given that the curves pressure versus mass flow for each rotor are very close, the power value shows little information about the improvement.

The torque curve has the same shape that the power one $($ Power $=$ Torque Rotation $)$. Thus, a torque comparison is more suitable, given that the points are collected for different rotations.

The chart (Figure 8.19) shows that both optimized rotors have better value for the torque for almost all values of mass flow. Again interpolating the curves (Eq.8.3) it is possible to compare the values in an equal basis.

$$
\begin{array}{ll}
y=0,00010 x^{2}+0,00013 x+0.00000 & \text { Straight Blade } \\
y=0,00009 x^{2}+0,00010 x+0.00004 & \text { Optimized }\left(w_{e}=1.0\right) \\
y=0,00008 x^{2}+0,00014 x+0.00001 & \text { Optimized }\left(w_{e}=0.7, w_{v}=0.3\right)
\end{array}
$$

Table 8.7 shows the interpolated values for the three rotors, and has a column with the percentage change from the original rotor. It is possible to notice that the optimized rotor for energy dissipation $\left(w_{e}=1.0\right.$ - Figure 6.3a) has an average torque decrease of $10 \%$, while the rotor optimized for energy dissipation and vorticity $\left(w_{e}=0.7, w_{v}=0.3\right.$ - Figure 6.3b has an average torque decrease of $12 \%$ when compared with the straight blade rotor. This can be explained due to the higher pressure on the straight blade rotor and not necessarily due to the topology. 
Figure 8.19 - Comparison of computational torque for the three rotors.

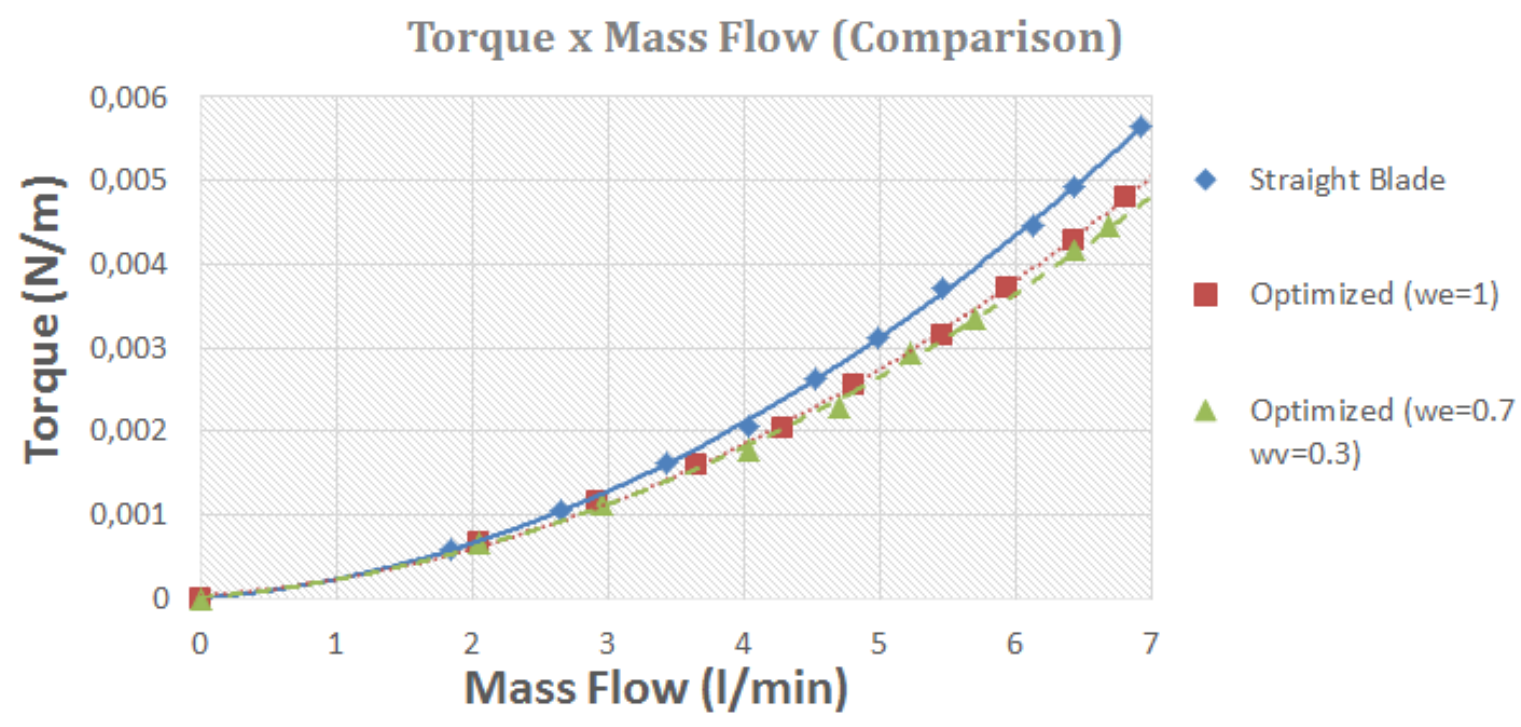

Table 8.7 - Interpolated values for torque.

\begin{tabular}{|c|c|c|c|c|c|}
\hline \multicolumn{6}{|c|}{ Torque } \\
\hline Mass Flow & Straight Blade & \multicolumn{2}{|c|}{ Optimized $\left(w_{e}=1\right)$} & \multicolumn{2}{|c|}{ Optimized $\left(w_{e}=0.7, w_{v}=0.3\right)$} \\
\hline$[l / \min ]$ & Value & Value & $\%$ change & Value & $\%$ change \\
\hline 1 & $2.32 \cdot 10^{-4}$ & $2.30 \cdot 10^{-4}$ & $-1 \%$ & $2.27 \cdot 10^{-4}$ & $-2 \%$ \\
\hline 2 & $6.61 \cdot 10^{-4}$ & $5.95 \cdot 10^{-4}$ & $-10 \%$ & $6.00 \cdot 10^{-4}$ & $-9 \%$ \\
\hline 3 & $1.29 \cdot 10^{-3}$ & $1.14 \cdot 10^{-3}$ & $-12 \%$ & $1.13 \cdot 10^{-3}$ & $-12 \%$ \\
\hline 4 & $2.11 \cdot 10^{-3}$ & $1.85 \cdot 10^{-3}$ & $-12 \%$ & $1.81 \cdot 10^{-3}$ & $-14 \%$ \\
\hline 5 & $3.13 \cdot 10^{-3}$ & $2.74 \cdot 10^{-3}$ & $-12 \%$ & $2.66 \cdot 10^{-3}$ & $-15 \%$ \\
\hline 6 & $4.34 \cdot 10^{-3}$ & $3.81 \cdot 10^{-3}$ & $-12 \%$ & $3.66 \cdot 10^{-3}$ & $-16 \%$ \\
\hline 7 & $5.75 \cdot 10^{-3}$ & $5.05 \cdot 10^{-3}$ & $-12 \%$ & $4.81 \cdot 10^{-3}$ & $-16 \%$ \\
\hline Average & & & $-10 \%$ & & $-12 \%$ \\
\hline
\end{tabular}




\section{CONCLUSION}

The complete development cycle of designing a radial flow machine by using the topology optimization is performed, aiming to increase the overall performance and decrease the vorticity. In order to verify the effectiveness of this approach, a steady state model of a rotary flow machine is implemented and a small scale prototype is built and characterized.

The numerical results show that non-intuitive topologies can be obtained by setting different objective functions with various weighting coefficients. It is noticeable that vorticity can be minimized by establishing a domain with intermediary porosity around the outlet. However, this domain can not be built.

Furthermore, the FEniCS environment jointly with the Dolfin-Adjoint package have shown to be an effective approach for implementing the topology optimization of fluid flow problems in a rotary domain. Also, the IPOpt optimizer has proved to be suitable for the solution of the proposed optimization problems.

The proposed process to design radial flow machine rotors was successfully implemented and is composed of: definition of the pump requirements; solution of the FEM model; performing of the topology optimization; post-processing of the results by using splines; creation of the final design in a CAD software; 3D printing of the prototype; testing of the final prototype and verification of the experimental-computational discrepancy.

The studied example of the straight blade optimization shows that the experimental results are coherent with the computational model, indicating that analysis done in the model would represent the prototype behavior. Also, the optimization shows that the optimized rotors have better values for the objective functions. The rotor designed for energy dissipation minimization presented an average improvement in the order of $68 \%$ for the energy dissipation functional and $62 \%$ for the vorticity functional.

The pump power optimization results have topologies very similar to the pump energy dissipation results. Thus, the final topologies resulting of the post-process are almost identical. Hence, rotors of the power optimization results were not built.

The turbine topologies for power optimization showed improvements in the power value, however, the optimized topologies have higher value for energy dissipation and vorticity.

The simulation indicates that the pump with a volute have similar velocity and pressure profiles among the blades. Also, the volute promotes less fluid recirculation and 
consequently presents lower energy dissipation and vorticity.

\subsection{Future Work}

Based on the aforementioned conclusions the author suggests that future work should focus on using real fluid properties and real dimensions on the optimization phase. Also, on performing the optimization by using 3D models.

Besides, considering other applications it is important to include models that consider high-Reynolds numbers, turbulence models and non-newtonian flows. For example, in blood pumps it is interesting to include a non-newtonian fluid model and to define other objective functions that are more relevant for this specific case, such as, hemolysis and wall shear stress. 


\section{REFERENCES}

AAROnSON, K. D.; SLAUGHTER, M. S.; MILLER, L. W.; MCGEE, E. C.; COTTS, W. G.; ACKER, M. a.; JESSUP, M. L.; GREGORIC, I. D.; LOYALKA, P.; FRAZIER, O. H.; JEEVANANDAM, V.; ANDERSON, A. S.; KORMOS, R. L.; TEUTEBERG, J. J.; LEVY, W. C.; NAFTEL, D. C.; BITTMAN, R. M.; PAGANI, F. D.; HATHAWAY, D. R.; BOYCE, S. W. Use of an Intrapericardial, Continuous-Flow, Centrifugal Pump in Patients Awaiting Heart Transplantation. Circulation, v. 125, n. 25, p. 3191-3200, jun 2012. ISSN 0009-7322. Available from Internet: $<$ http://circ.ahajournals.org/cgi/doi/10.1161/CIRCULATIONAHA.111.058412>. Cited in page 18 .

ABRAHAM, F.; BEHR, M.; HEINKENSCHLOSS, M. The effect of stabilization in finite element methods for the optimal boundary control of the Oseen equations. Finite Elements in Analysis and Design, v. 41, n. 3, p. 229-251, 2004. ISSN 0168874X. Cited in page 37 .

ALNAES, M.; LOGG, A.; MARDAL, K. A.; SKAVHAUG, O.; LANGTANGEN, H. Unified framework for finite element assembly. International Journal of Computational Science and Engineering, v. 4, n. 4, p. 231, may 2009. ISSN 1742-7185. Available from Internet: <http://arxiv.org/abs/1205.3039http://dx.doi.org/10.1504/IJCSE.2009. 029160http://www.inderscience.com/link.php?id=29160>. Cited in page 51.

ALNÆS, M. S.; LOGG, A.; ØLGAARD, K. B.; ROGNES, M. E.; WELLS, G. N. Unified form language. ACM Transactions on Mathematical Software, v. 40, n. 2, p. 1-37, mar 2014. ISSN 00983500. Available from Internet: <http://arxiv.org/abs/1005.3014http: //arxiv.org/abs/1211.4047http://dl.acm.org/citation.cfm?doid=2594412.2566630>. Cited in page 51 .

AMESTOY, P. R.; DUFF, I. S.; L'EXCELLENT, J.-Y.; KOSTER, J. A Fully Asynchronous Multifrontal Solver Using Distributed Dynamic Scheduling. SIAM Journal on Matrix Analysis and Applications, v. 23, n. 1, p. 15-41, jan 2001. ISSN 0895-4798. Available from Internet: <http://epubs.siam.org/doi/abs/10.1137/S0895479899358194>. Cited in page 51 .

BALONI, B. D.; PATHAK, Y.; CHANNIWALA, S. Centrifugal blower volute optimization based on Taguchi method. Computers \& Fluids, Elsevier Ltd, v. 112, n. February, p. 72-78, may 2015. ISSN 00457930. Available from Internet: <http://linkinghub.elsevier.com/retrieve/pii/S0045793015000444>. Cited in page 19 .

BORRVALL, T.; PETERSSON, J. Topology optimization of fluids in Stokes flow. International Journal for Numerical Methods in Fluids, v. 41, n. 1, p. 77-107, jan 2003. ISSN 0271-2091. Available from Internet: <http://onlinelibrary.wiley.com/doi/ 10.1002/fld.426/abstracthttp://doi.wiley.com/10.1002/fld.426>. Cited 5 times in pages 26, 31, 34, 35, and 36.

CASAS, V.; PENA, F.; DURO, R. Automatic Design and Optimization of Wind Turbine Blades. In: 2006 International Conference on Computational Inteligence for Modelling Control and Automation and International Conference 
on Intelligent Agents Web Technologies and International Commerce (CIMCA'06). IEEE, 2006. p. 205-205. ISBN 0-7695-2731-0. Available from Internet: $<$ http://ieeexplore.ieee.org/lpdocs/epic03/wrapper.htm?arnumber $=4052821>$. Cited in page 19 .

DENG, Y.; LIU, Z.; WU, J.; WU, Y. Topology optimization of steady Navier-Stokes flow with body force. Computer Methods in Applied Mechanics and Engineering, Elsevier B.V., v. 255, p. 306-321, mar 2013. ISSN 00457825. Available from Internet: <http://linkinghub.elsevier.com/retrieve/pii/S0045782512003532>. Cited in page 32 .

DERAKHSHAN, S.; POURMAHDAVI, M.; ABDOLAHNEJAD, E.; REIHANI, A.; OJAGHI, A. Numerical shape optimization of a centrifugal pump impeller using artificial bee colony algorithm. Computers \& Fluids, v. 81, p. 145-151, 2013. ISSN 00457930. Available from Internet: <http://linkinghub.elsevier.com/retrieve/pii/ S0045793013001588>. Cited 2 times in pages 19 and 21.

EVGRAFOV, A. Topology optimization of slightly compressible fluids.

Doktorsavhandlingar vid Chalmers Tekniska Hogskola, v. 62, n. 1, p. 55-81, 2004. ISSN 0346718X. Cited in page 31.

EVGRAFOV, A. The Limits of Porous Materials in the Topology Optimization of Stokes Flows. Applied Mathematics and Optimization, v. 52, n. 3, p. 263-277, oct 2005. ISSN 0095-4616. Available from Internet: <http://link.springer.com/10.1007/ s00245-005-0828-z>. Cited in page 31 .

FARRELL, P. E.; HAM, D. A.; FUNKE, S. W.; ROGNES, M. E. Automated Derivation of the Adjoint of High-Level Transient Finite Element Programs.

SIAM Journal on Scientific Computing, v. 35, n. 4, p. C369-C393, jan 2013.

ISSN 1064-8275. Available from Internet: <http://arxiv.org/abs/1204.5577http: //epubs.siam.org/doi/abs/10.1137/120873558>. Cited 2 times in pages 43 and 52 .

FOX, R. W.; PRITCHARD, P. J.; MCDONALD, A. T. Introduction to Fluid Mechanics. 7. ed. [S.l.]: Wiley, 2008. 768 p. ISBN 0471742996. Cited in page 37.

FRASER, W. H. Flow Recirculation in Centrifugal Pumps. [S.l.]: TENTH TURBOMACHINERY SYMPOSIUM, 2001. Cited in page 36.

FUNKE, S. W.; FARRELL, P. E. A framework for automated PDE-constrained optimisation. arXiv preprint arXiv:1302.3894, feb 2013. Available from Internet: <http://arxiv.org/abs/1302.3894>. Cited in page 52 .

GERSBORG-HANSEN, A. Topology optimization of incompressible Newtonian flows at moderate Reynolds numbers. Tese (Doutorado), 2003. Cited 2 times in pages 31 and 34 .

GERSBORG-HANSEN, a.; SIGMUND, O.; HABER, R. Topology optimization of channel flow problems. Structural and Multidisciplinary Optimization, v. 30, n. 3, p. 181-192, sep 2005. ISSN 1615-147X. Available from Internet: <http://link.springer.com/10.1007/s00158-004-0508-7>. Cited in page 31.

GÖLCÜ, M.; PANCAR, Y.; SEKMEN, Y. Energy saving in a deep well pump with splitter blade. Energy Conversion and Management, v. 47, n. 5, p. 638-651, mar 2006. ISSN 01968904. Available from Internet: <http: 
//linkinghub.elsevier.com/retrieve/pii/S0196890405001287> Cited 2 times in pages 20 and 58 .

HANSEN, M. O. L. Aerodynamics of Wind Turbines. 2. ed. [S.l.]: Routledge, 2007. 208 p. ISBN 978-1844074389. Cited in page 19.

JAFARZADEH, B.; HAJARI, A.; ALISHAHI, M.; AKBARI, M. The flow simulation of a low-specific-speed high-speed centrifugal pump. Applied Mathematical

Modelling, v. 35, n. 1, p. 242-249, jan 2011. ISSN 0307904X. Available from Internet: <http://linkinghub.elsevier.com/retrieve/pii/S0307904X10002271>. Cited in page 18 .

KOGA, A. A. Projeto de Dispositivos de Microcanais Utilizando o Método de Otimização Topológica. 2010. Cited 2 times in pages 21 and 23.

KUNDU, P. K.; COHEN, I. M.; DOWLING, D. R. Fluid mechanics. 5. ed. [S.l.]: Academic Press, 2013. 920 p. ISBN 9780123821003. Cited 2 times in pages 26 and 27.

LEE, Y.-T.; AHUJA, V.; HOSANGADI, A.; SLIPPER, M. E.; MULVIHILL, L. P.; BIRKBECK, R.; COLEMAN, R. M. Impeller Design of a Centrifugal Fan with Blade Optimization. International Journal of Rotating Machinery, v. 2011, p. 1-16, 2011. ISSN 1023-621X. Available from Internet: <http://www.hindawi.com/journals/ijrm/2011/ $537824 />$. Cited in page 18 .

LOGG, A.; WELLS, G. N. DOLFIN. ACM Transactions on Mathematical Software, v. 37, n. 2, p. 1-28, apr 2010. ISSN 00983500. Available from Internet: $<$ http://portal.acm.org/citation.cfm?doid $=1731022.1731030>$. Cited in page 51 .

LOGG, A.; WELLS, G. N.; BOOK, T. F. Automated Solution of Differential Equations by the Finite Element Method. Berlin, Heidelberg: Springer Berlin Heidelberg, 2012. v. 84. ISBN 978-3-642-23098-1. Available from Internet: <http://link.springer.com/10.1007/978-3-642-23099-8>. Cited in page 51 .

MONTEVECCHI, F.; INZOLI, F.; REDAELLI, A.; MAMMANA, M. Preliminary Design and Optimization of an ECC Blood Pump by Means of a Parametric Approach. Artificial Organs, v. 19, n. 7, p. 685-690, jul 1995. ISSN 0160564X. Available from Internet: <http://doi.wiley.com/10.1111/j.1525-1594.1995.tb02403.x>. Cited in page 20

MUNSON, B. R.; ROTHMAYER, A. P.; OKIISHI, T. H.; HUEBSCH, W. W. Fundamentals of Fluid Mechanics. 7. ed. [S.l.]: John Wiley \& Sons, Inc., 2012. 792 p. ISBN 9781118116135. Cited in page 77

OLESEN, L. H.; OKKELS, F.; BRUUS, H. A high-level programming-language implementation of topology optimization applied to steady-state Navier-Stokes flow. International Journal for Numerical Methods in Engineering, v. 65, n. 7, p. 975-1001, feb 2006. ISSN 0029-5981. Available from Internet: <http://doi.wiley.com/10.1002/nme.1468>, Cited 2 times in pages 31 and 70.

PINGEN, G.; MAUTE, K. Optimal design for non-Newtonian flows using a topology optimization approach. Computers \& Mathematics with Applications, Elsevier Ltd, v. 59, n. 7, p. 2340-2350, apr 2010. ISSN 08981221. Available from Internet: <http://dx.doi.org/10.1016/j.camwa.2009.08.044http://linkinghub.elsevier.com/retrieve/ pii/S0898122109006336>. Cited in page 31. 
QUARTERONI, A.; ROZZA, G. Optimal Control and Shape Optimization of Aorto-Coronaric Bypass Anastomoses. Mathematical Models and Methods in Applied Sciences, v. 13, n. 12, p. 1801-1823, 2003. ISSN 0218-2025. Cited in page 37 .

ROMERO, J. S.; SILVA, E. C. N. A topology optimization approach applied to laminar flow machine rotor design. Comput. Methods Appl. Mech. Engrg, v. 279, p. 268-300, 2014. Available from Internet: <www.elsevier.com/locate/cma $>$. Cited 16 times in pages 11, 23, 27, 28, 32, 38, 39, 40, 44, 46, 47, 48, 56, 65, 103, and 105 ,

SLAUGHTER, M. S.; PAGANI, F. D.; MCGEE, E. C.; BIRKS, E. J.; COTTS, W. G.; GREGORIC, I.; Howard Frazier, O.; ICENOGLE, T.; NAJJAR, S. S.; BOYCE, S. W.; ACKER, M. a.; JOHN, R.; HATHAWAY, D. R.; NAJARIAN, K. B.; AARONSON, K. D. HeartWare ventricular assist system for bridge to transplant: Combined results of the bridge to transplant and continued access protocol trial. The Journal of Heart and Lung Transplantation, Elsevier, v. 32, n. 7, p. 675-683, jul 2013. ISSN 10532498. Available from Internet: <http://dx.doi.org/10.1016/j.healun.2013.04.004http: //linkinghub.elsevier.com/retrieve/pii/S1053249813012084>. Cited in page 18.

STEIN, W.; JOYNER, D. SAGE: System for Algebra and Geometry Experimentation* . ACM SIGSAM Bulletin, Timely Communication, v. 39, n. 2, 2005. Available from Internet: <http://sigsam.org/bulletin/articles/152/sage.pdf $>$. Cited in page 52 .

SVANBERG, K. The method of moving asymptotes - a new method for structural optimization. International Journal for Numerical Methods in Engineering, v. 24, n. 2, p. 359-373, feb 1987. ISSN 0029-5981. Available from Internet: <http://doi.wiley.com/10.1002/nme.1620240207>. Cited in page 53 .

WÄCHTER, A. An Interior Point Algorithm for Large-Scale Nonlinear Optimization with Applications in Process Engineering. Tese (Doutorado), 2002. Cited in page 53 .

WÄCHTER, A. Short Tutorial: Getting Started With Ipopt in 90 Minutes. In: TOLEDO, U. N.; SCHENK, O.; SIMON, H. D.; SIVAN (Ed.). Combinatorial Scientific Computing. Dagstuhl, Germany: Schloss Dagstuhl - Leibniz-Zentrum fuer Informatik, Germany, 2009. ISBN 1862-4405. ISSN 1862-4405. Available from Internet: <http://drops.dagstuhl.de/opus/volltexte/2009/2089>. Cited in page 53.

WÄCHTER, A.; BIEGLER, L. T. On the implementation of an interior-point filter line-search algorithm for large-scale nonlinear programming. Mathematical Programming, v. 106 , n. 1, p. 25-57, mar 2006. ISSN 0025-5610. Available from Internet: <http://link.springer.com/10.1007/s10107-004-0559-y>. Cited in page 53.

WANG, L.; YANG, Y.; DONG, C.; MOROSUK, T.; TSATSARONIS, G. Parametric optimization of supercritical coal-fired power plants by MINLP and differential evolution. Energy Conversion and Management, Elsevier Ltd, v. 85, p. 828-838, 2014. ISSN 01968904. Available from Internet: <http://dx.doi.org/10.1016/j.enconman.2014.01.006>. Cited in page 20.

WEN-GUANG, L. Inverse Design of Impeller Blade of Centrifugal Pump with a Singularity Method. Jordan Journal of Mechanical and Industrial ..., v. 5, n. 2, p. 119-128, 2011. ISSN 19956665. Cited in page 19. 
WHITE, F. Fluid Mechanics. 7. ed. [S.l.]: McGraw-Hill Science/Engineering/Math, 2010. 862 p. ISBN 0077422414. Cited in page 25.

WIKER, N.; KLARBRING, A.; BORRVALL, T. Topology optimization of regions of Darcy and Stokes flow. International Journal for Numerical Methods in Engineering, v. 69, n. July 2006, p. 1374-1404, 2007. ISSN 00295981. Cited in page 31

YU, S.; NG, B.; CHAN, W.; CHUA, L. The flow patterns within the impeller passages of a centrifugal blood pump model. Medical Engineering \& Physics, v. 22, n. 6, p. 381-393, jul 2000. ISSN 13504533. Available from Internet:

<http://linkinghub.elsevier.com/retrieve/pii/S135045330000045X>. Cited in page 18 .

ZHU, B.; ZHANG, X.; FATIKOW, S. A multi-objective method of hinge-free compliant mechanism optimization. Structural and Multidisciplinary Optimization, v. 49, n. 3, p. 431-440, 2014. ISSN 16151488. Cited 2 times in pages 39 and 44.

ZIENKIEWICZ, O. C.; TAYLOR, R. L.; ZHU, J. The Finite Element Method: Its Basis and Fundamentals, Seventh Edition. 7. ed. [S.1.]: Butterworth-Heinemann, 2013. 756 p. ISBN 1856176339. Cited in page 25. 


\section{APPENDIX A - FE RESULTS VERIFICATION}

\section{Simulation Parameters}

This section presents some results obtained by using the formulation of Section 2.3 applied to different geometries, such as straight blades and curved blades. Two types of fluids are used, a fluid with arbitrary properties and water. The fluids and their properties are given by Table A.1.

Table A.1 - Fluid Properties.

\begin{tabular}{ccc}
\hline Material & Density $\left[\mathrm{kg} / \mathrm{m}^{3}\right]$ & Viscosity $\left[N \cdot \mathrm{s} / \mathrm{m}^{3}\right]$ \\
\hline Fluid 1 & 1.0 & 0.1 \\
Water & 997.0 & 0.001 \\
\hline
\end{tabular}

\section{Straight Blade FEM}

The first test aims to verify the FEM formulation. Thus, a straight blade model with arbitrary dimensions is defined and Fluid 1 (Table A.1)is used as fluid. The model used has an inner radius of $0.4[\mathrm{~m}]$, outer radius of $1.0[\mathrm{~m}]$ and an angle of $45^{\circ}$. The velocity imposed in the fluid entrance is normal to the boundary and has module equal to 1.0. The fluid exit has a relative pressure of zero and the remaining walls have a no-slip condition $(u=0)$. The entire domain is subjected to rotation $\omega_{z}$. The dimensions are shown in Figure A.1 (a) and the boundary conditions can be seen in Figure A.1 (b) and Table A.2.

Figure A.1 - Straight Blade: (a)Dimensions and (b)Boundary conditions.
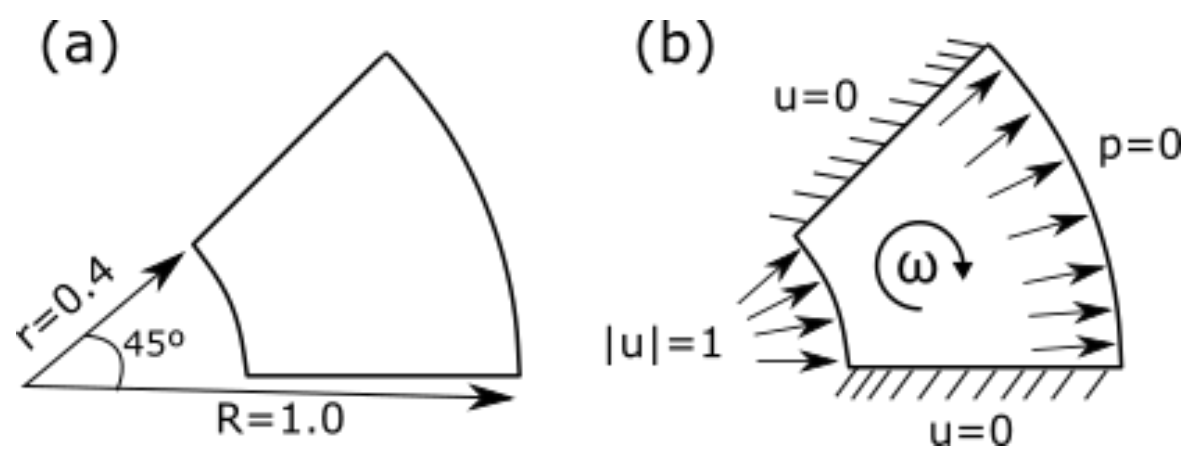
Table A.2 - Straight Blade Boundary Conditions.

\begin{tabular}{ccl}
\hline Condition & Value & \\
\hline$\left|u_{\text {in }}\right|$ & 1.0 & {$\left[\mathrm{~m} / \mathrm{s}^{2}\right]$} \\
$p_{\text {out }}$ & 0.0 & {$[\mathrm{~Pa}]$} \\
$u_{\text {wall }}$ & 0.0 & {$\left[\mathrm{~m} / \mathrm{s}^{2}\right]$} \\
$\omega_{z}$ & 1000.0 & {$[\mathrm{rpm}]$} \\
\hline
\end{tabular}

To implement the model in each software, ANSYS and FEniCS, it is necessary to define the geometry and then perform a mesh generation. ANSYS has an auto-meshing tool that optimizes the mesh accordingly to the geometry, so it builds a square mesh, whereas, in FEniCS the mesh generator can only construct triangular meshes. The resulting mesh for each case is shown in Figures A.2a and A.2b. With the meshes defined it is possible to solve the problem by considering the boundary conditions of Figure A.1(b). Thus, the FEM system equations can be solved in this domain in order to obtain the velocity and pressure fields. The results of this implementation for ANSYS and FEniCS are shown in Figure A.2.

In Figure A.2, it is possible to note that the results are coherent between both ANSYS and FEniCS implementations. The general format of the velocity and pressure fields are very similar and the numeric values are also very close to each other. However, the peak value of velocity is different, being higher in ANSYS. So, a further comparison is performed by exporting these velocity values to MATLAB ®and using a three-dimensional interpolation function to compare with the results presented by Romero and Silva (ROMERO; SILVA, 2014). This comparison is shown in Figure A.3.

There are differences between the results shown in Figure A.3. mostly around the fluid exit. The relative velocity fields from FEniCS and Romero are very similar showing small punctual divergences (Figure A.3b), due to the fact that the models are built by using different types of element (triangular and square). The pressure field comparison (Figure A.3f) presents even smaller divergences, given the magnitude order pressure (4500) and the maximum difference absolute value (65). ANSYS relative velocity field has a more representative difference (Figs. A.3c and A.3d). This can be justified due to the fact that ANSYS uses element stabilization methods that are not present in the other cases. 
Figure A.2 - Straight Blade: Mesh, Relative velocity and Pressure fields for ANSYS and FEniCS.

(a) Mesh (ANSYS).

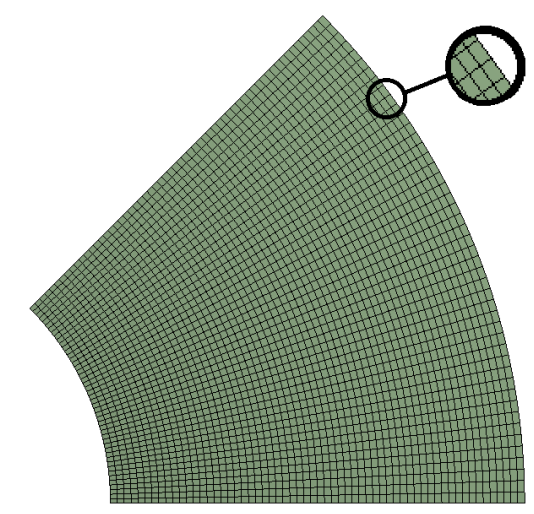

(c) Relative velocity Field (ANSYS).

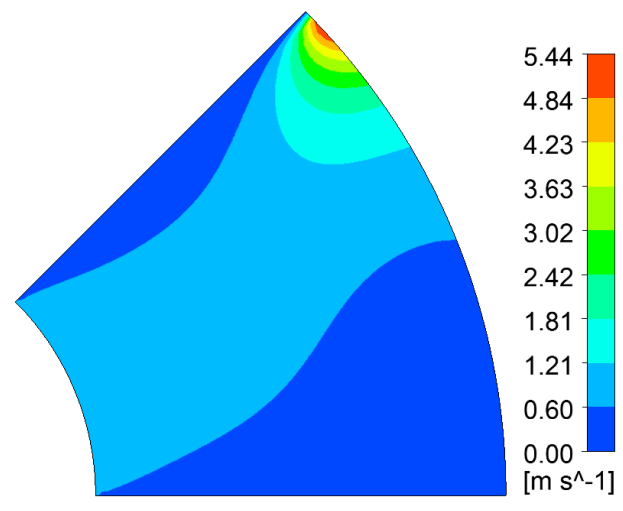

(e) Pressure Field (ANSYS).

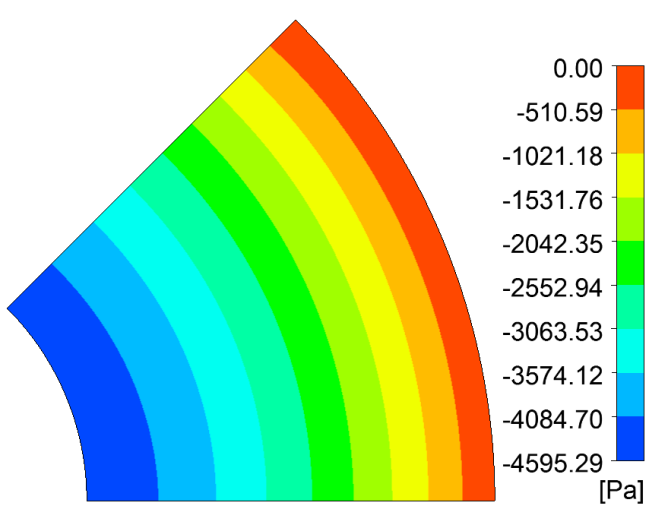

(b) Mesh (FEniCS).

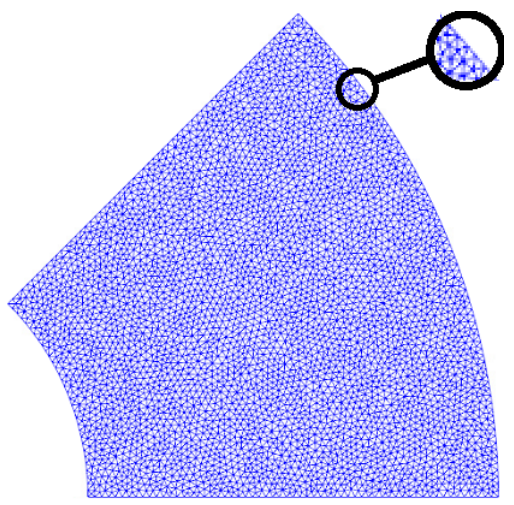

(d) Relative velocity Field (FEniCS).

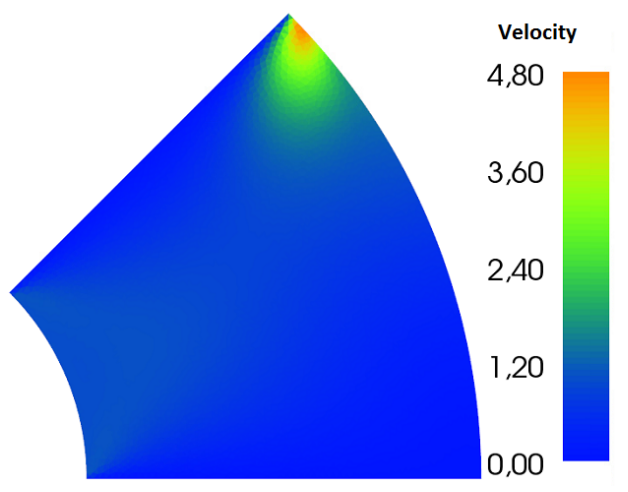

(f) Pressure Field (FEniCS).

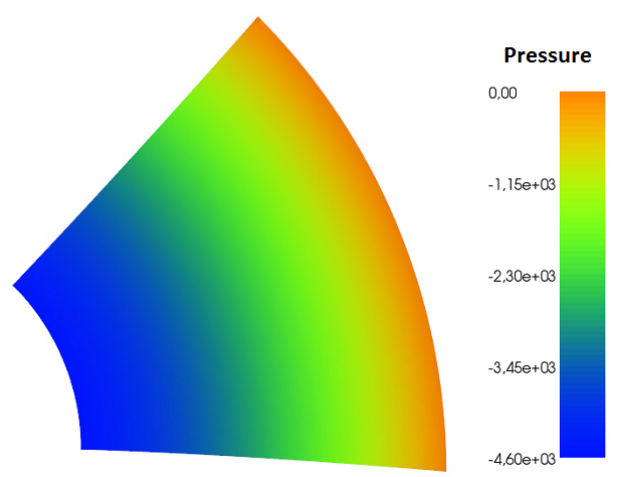


Figure A.3 - Straight Blade: Relative velocity and Pressure fields comparison between FEniCS, ANSYS and Romero and Silva (2014) results.

(a) Relative velocity field ROMERO; SILVA, 2014).

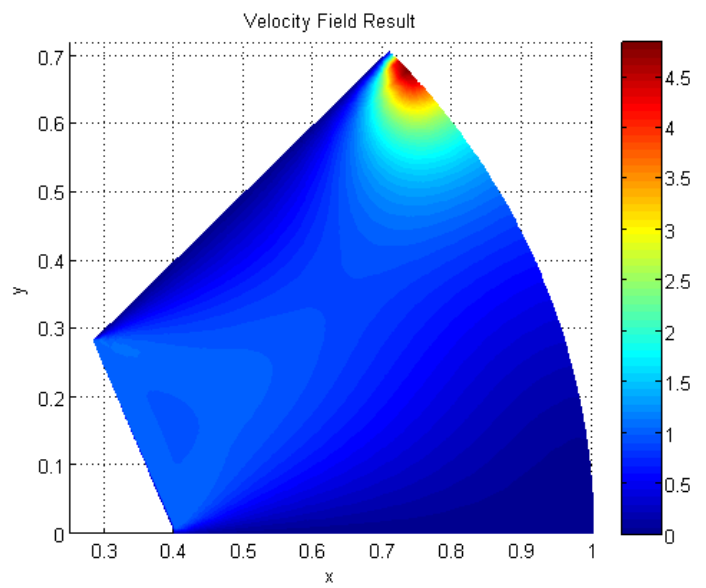

(c) Comparison relative velocity field (ANSYS Romero, 2014).

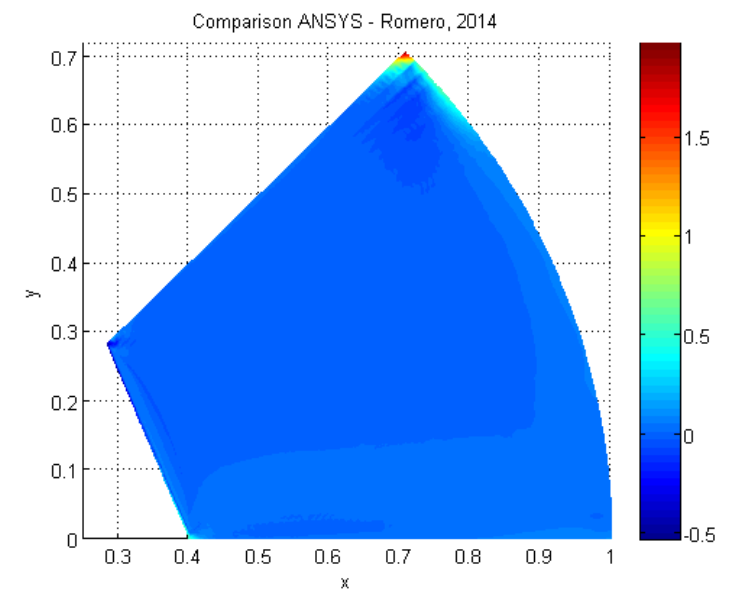

(e) Pressure field (ROMERO; SILVA, 2014).

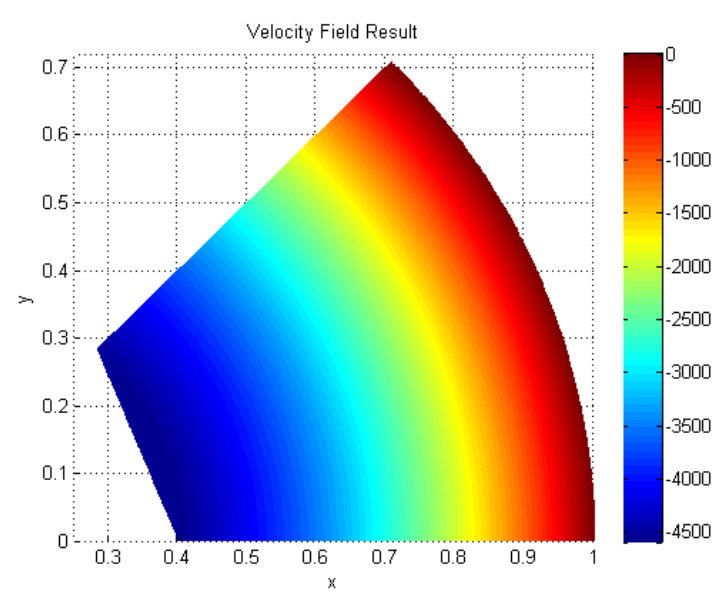

(b) Comparison relative velocity field (FEniCS Romero, 2014).

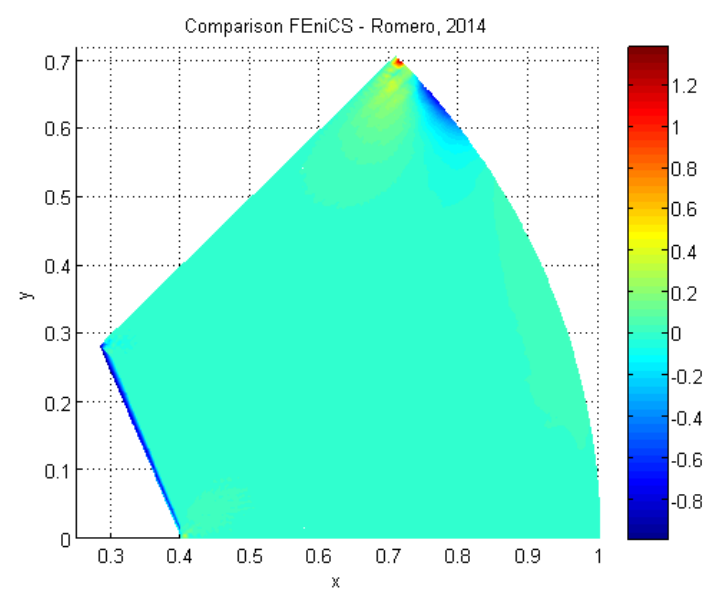

(d) Comparison relative velocity field (ANSYS FEniCS).

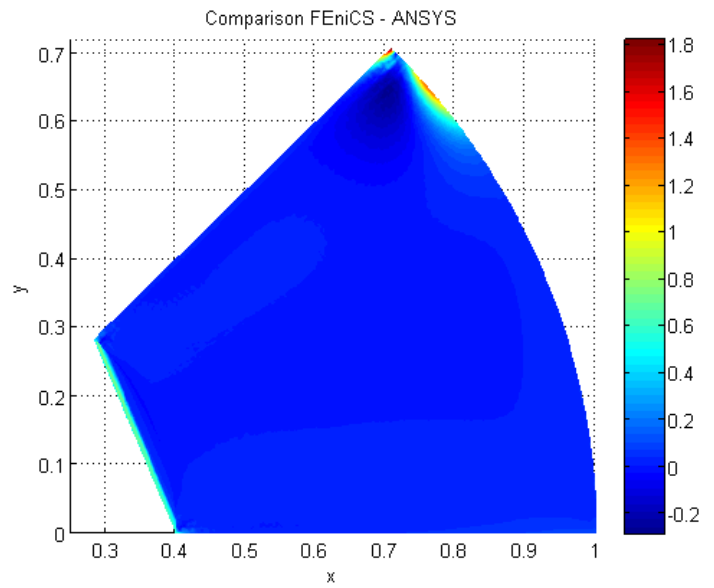

(f) Comparison pressure field (FEniCS - Romero, 2014).

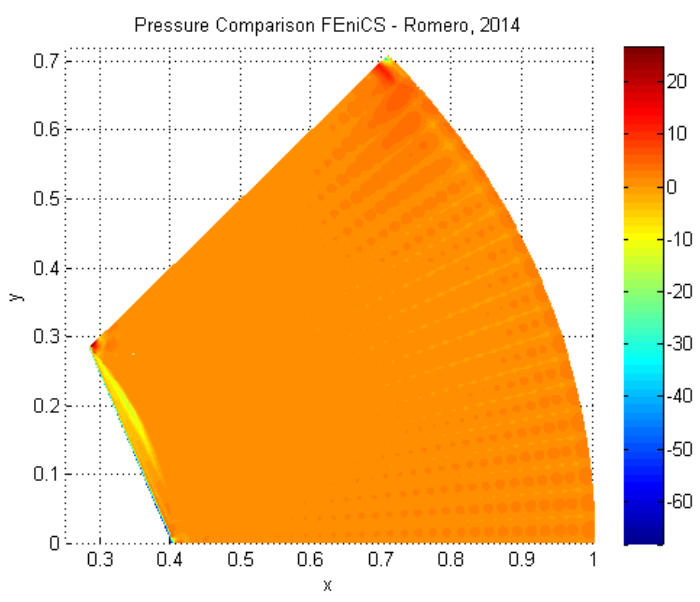




\section{Curved Blade FEM}

Another test is performed by using a Curved Blade geometry with the same conditions of the Straight Blade case, that is using a fictitious fluid (Table A.1), inner radius of $0.4[\mathrm{~m}]$ and outer radius of $1.0[\mathrm{~m}]$. Velocity in the fluid entrance is normal to the boundary and it has module equal to 1.0. The fluid exit has a relative pressure of zero and the remaining walls have a no-slip condition $(u=0)$. The entire domain is subjected to rotation $\omega_{z}$. Figure A.4 illustrates these conditions and the value of each condition is defined on Table A.2.

Figure A.4 - Curved Blade: (a)Dimensions and (b)Boundary conditions.

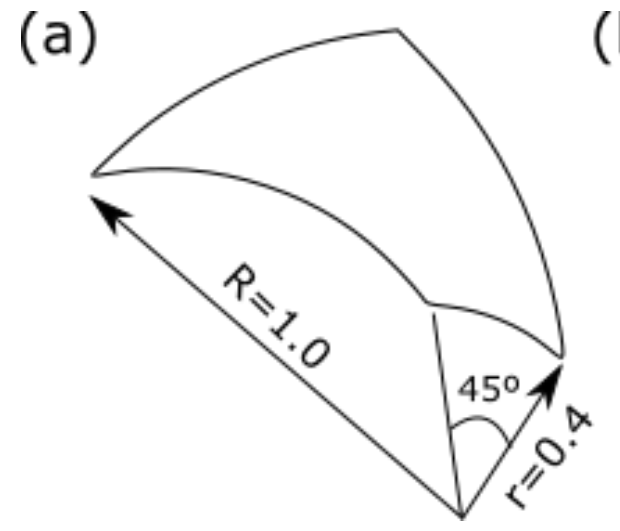

(b)

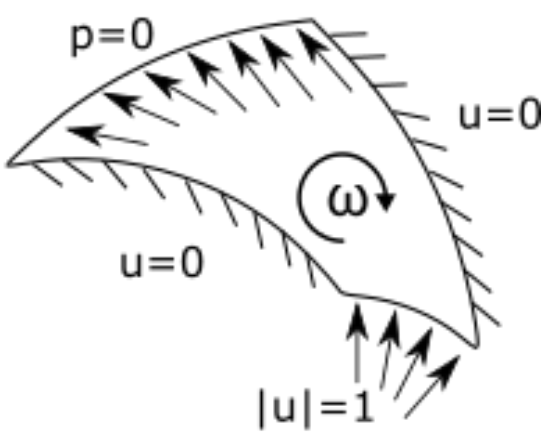

With the defined geometry the next step is mesh the model. As in the Straight Blade case, FEniCS model is meshed with triangles while ANSYS model is meshed with a square mesh. Thus, meshes, velocity and pressure fields for each case are shown in Figure A.5.

It is possible to notice, from Figure A.5, that the maximum velocity from FEniCS is lower than the one from ANSYS. In addition, the pressure gain, given by maximum pressure minus minimum pressure, from FEniCS and ANSYS are very close. To verify the divergences the results are exported to $M A T L A B$ (B)and interpolated with a convex function, then they are subtracted, as shown in Figures A.5e and A.5h The discrepancies in velocity and pressure fields can be attributed not only to the fact that different elements are used, but also to deviations on the geometry, because each software uses a different CAD to build the model, and so, the circumferences have contour deviations that influence the results. 
Figure A.5 - Curved Blade: Velocity and Pressure fields for ANSYS and FEniCS.

(a) Mesh (ANSYS).

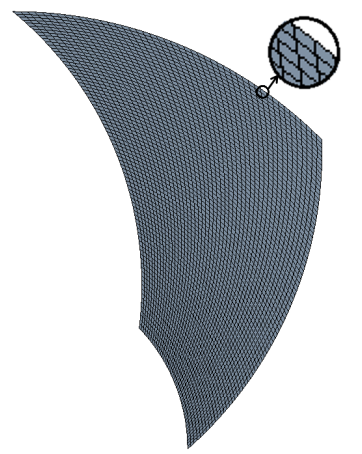

(b) Mesh (FEniCS).

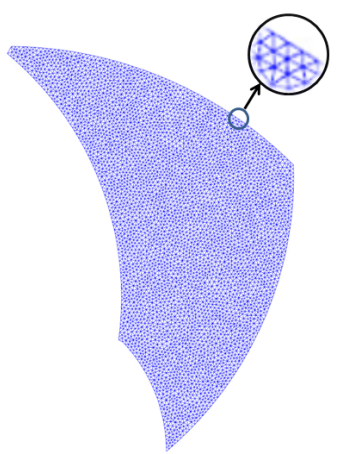

(c) Relative velocity Field (ANSYS).

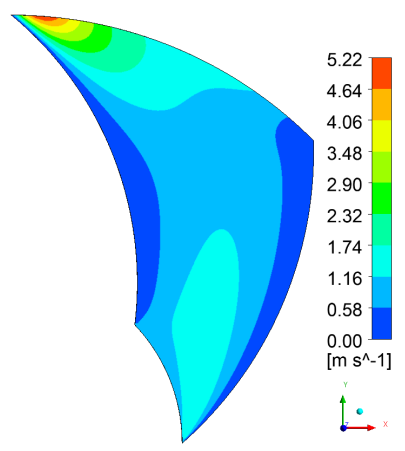

(f) Pressure Field (ANSYS).

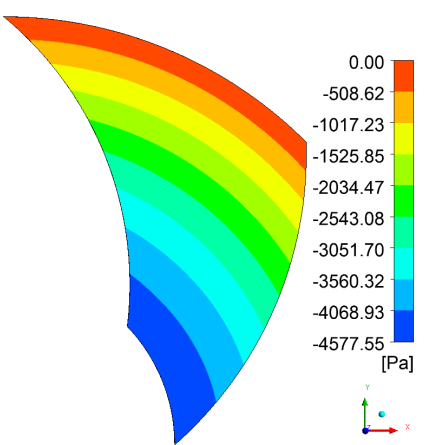

(d) Relative velocity Field (FEniCS).
(e) Relative velocity Comparison (ANSYS-FEniCS).
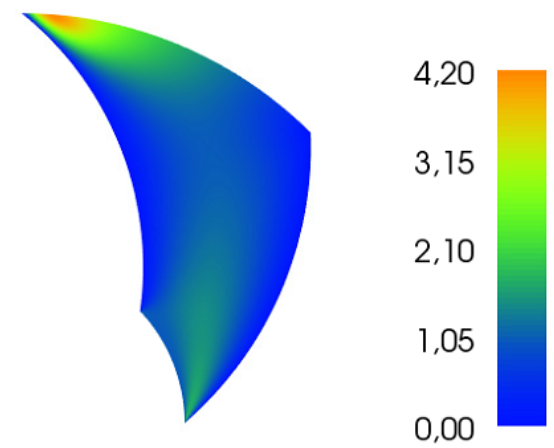

(h) Pressure Comparison

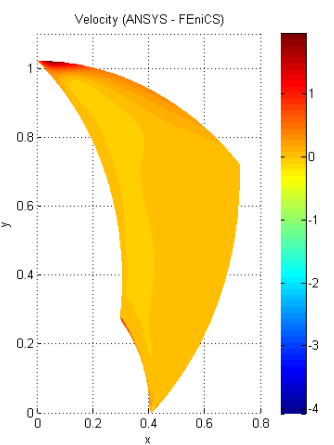

(g) Pressure Field (FEniCS).

\section{(ANSYS-FEniCS).}
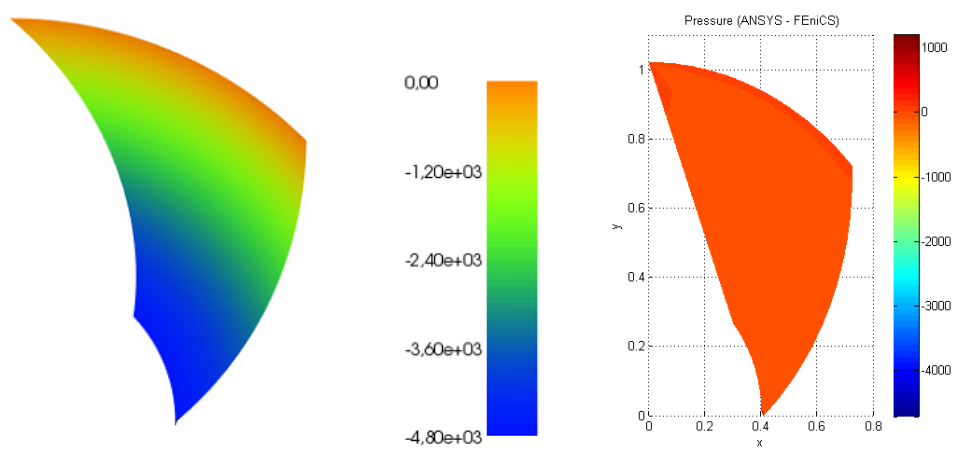


\section{APPENDIX B - WALL SHEAR STRESS ANALYSIS}

The Wall Shear Stress (WSS) has a strong correlation with the hemolysis rate. Thus, it is important for a blood pump rotor to have the smallest WSS possible. Hence, a comparison between the WSS of the analysed rotors is performed and presented bellow. The ANSYS uses the following WSS definition:

$$
\tau=\sqrt{\tau_{x}^{2}+\tau_{y}^{2}+\tau_{z}^{2}}
$$

where,

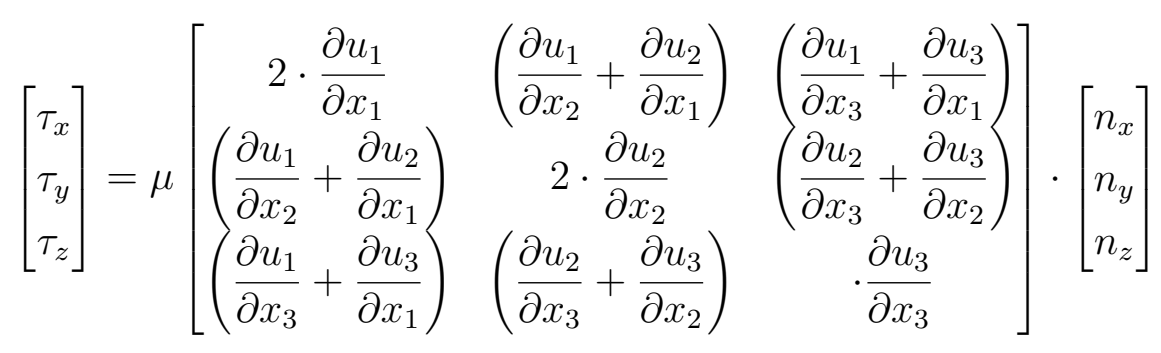

The WSS distribution is shown in Figures B.1, B.2 and B.3 for the straight blade, the optimized rotor for energy dissipation (Figure 6.3a) and the optimized rotor for energy dissipation and vorticity (Figure 6.3b), respectively. Observing the charts it is possible to notice that the straight blade rotor has a higher maximum WSS than the others. In general, it has higher values over the entire domain. The rotor views (Figs. B.1b, B.2b and B.3b) show that the WSS for the straight blade has almost double value of the optimized rotors.

Comparing the WSS of the optimized rotors we observe that the peak value is nearly the same, and the distribution is homogeneous between the blades at each rotor. Hence, the optimized rotors should present a lower hemolysis rate than the straight blade rotor. 
Figure B.1 - Wall shear stress distribution for straight blade rotor (Fig. 6.2a), 4.99[l/min] and $1920[$ rpm $]$.

(a) Top view.

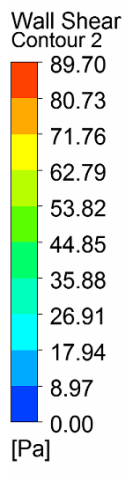

(b) Rotor view.

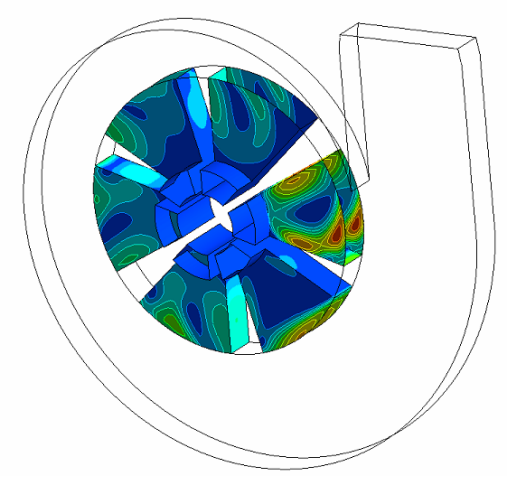

Figure B.2 - Wall shear stress distribution for optimized rotor for energy dissipation, (Fig. 6.3a), 4.81[l/min $]$ and $1923[\mathrm{rpm}]$.

(a) Top view.

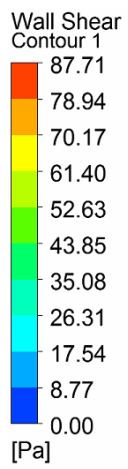

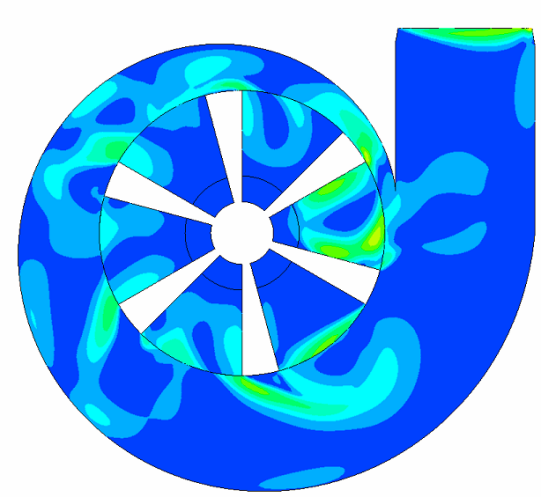

Wall Shear

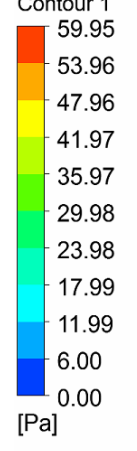

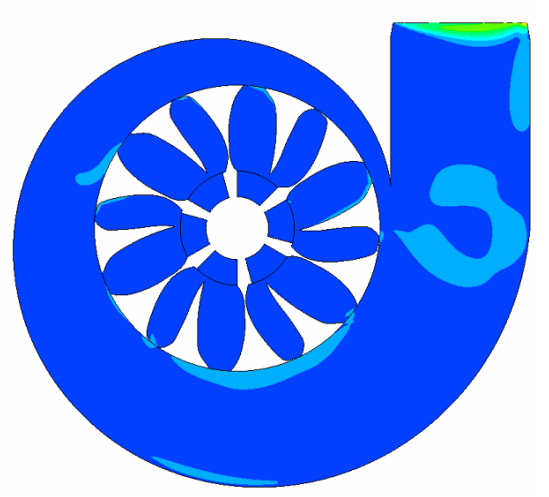

(b) Rotor view.

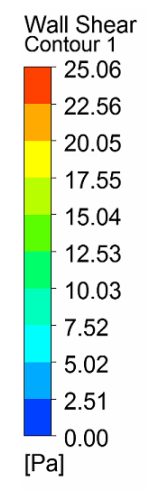

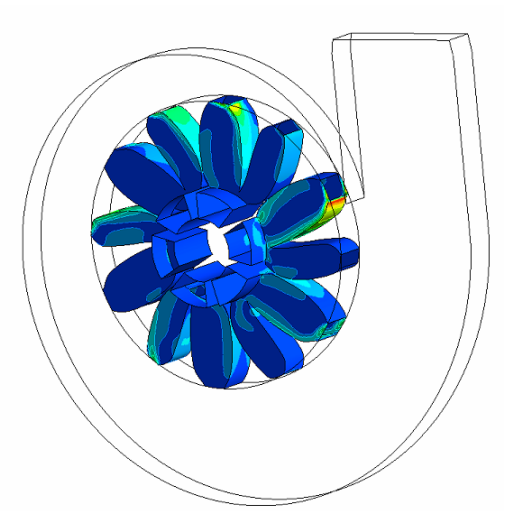

Figure B.3 - Wall shear stress distribution for optimized rotor for energy dissipation and vorticity (Fig. 6.3b), 4.69[l/min] and 1946 [rpm].

(a) Top view.

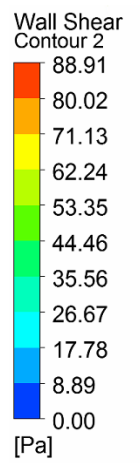

(b) Rotor view.

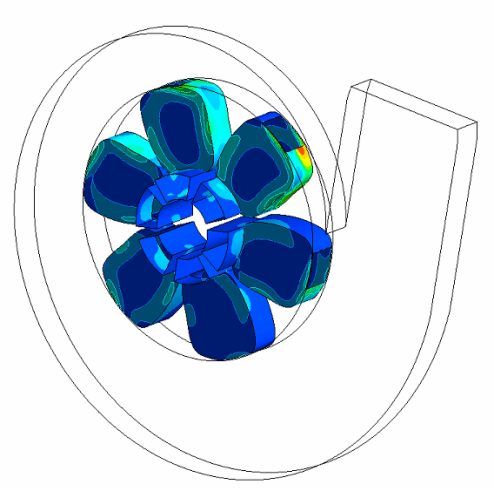




\section{APPENDIX C - TECHNICAL DRAWINGS}

Parts list:

(1) Pump case (with volute);

(2) Straight Blade Rotor;

(3) Optimized rotor for energy dissipation $\left(w_{e}=1.0\right)$;

(4) Optimized rotor for energy dissipation and vorticity $\left(w_{e}=0.7, w_{v}=0.3\right)$. 


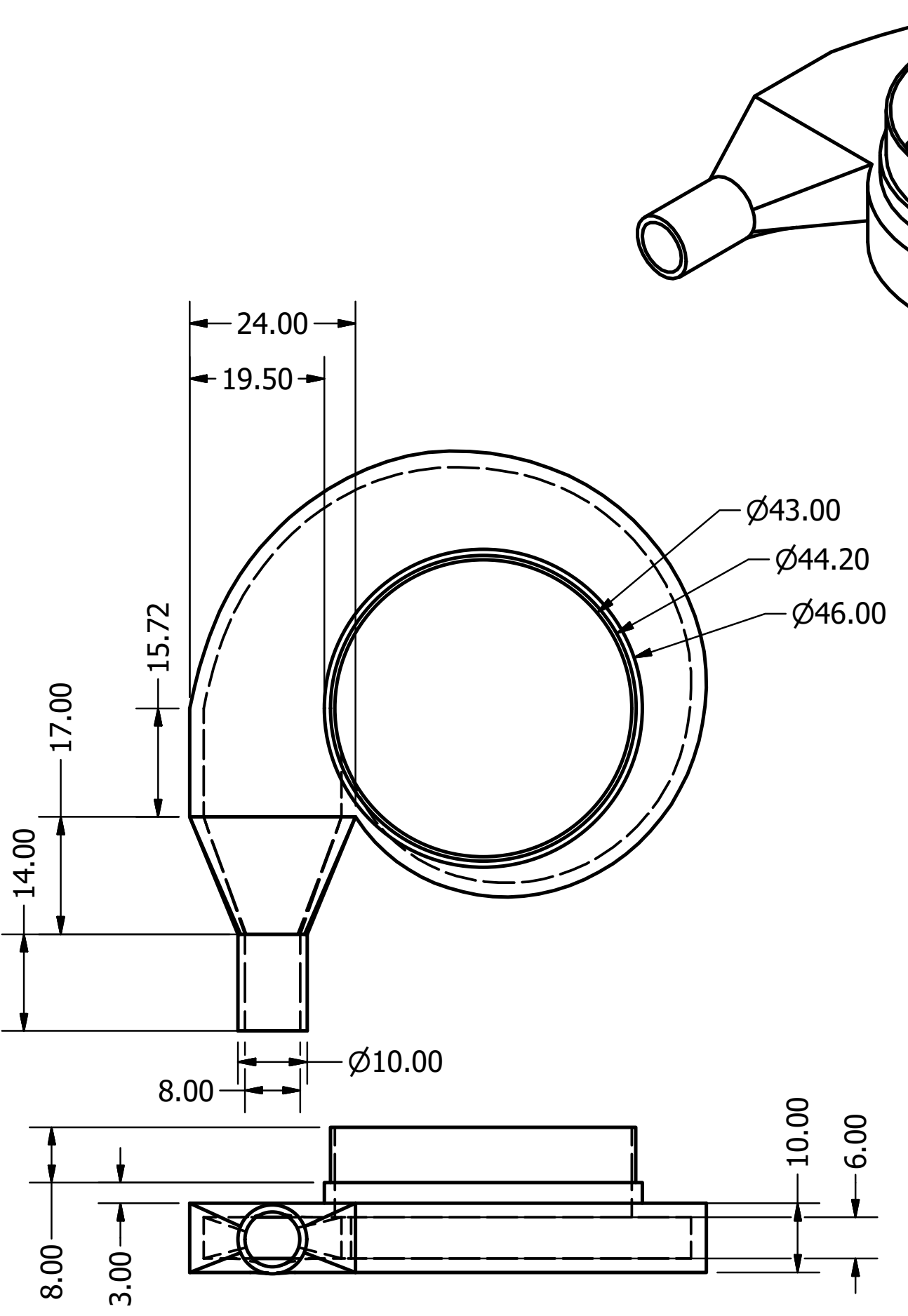

\begin{tabular}{|c|c|c|c|}
\hline $\begin{array}{l}\text { DRAWN } \\
\text { L } \\
\end{array}$ & & & \\
\hline CHECKED & & & \\
\hline$\overline{Q A}$ & TITLE & & \\
\hline MFG & Pum & & \\
\hline APPROVED & & & \\
\hline & SIZE & DWG NO & REV \\
\hline & A4 & lcase & \\
\hline & SCALE & & \\
\hline
\end{tabular}




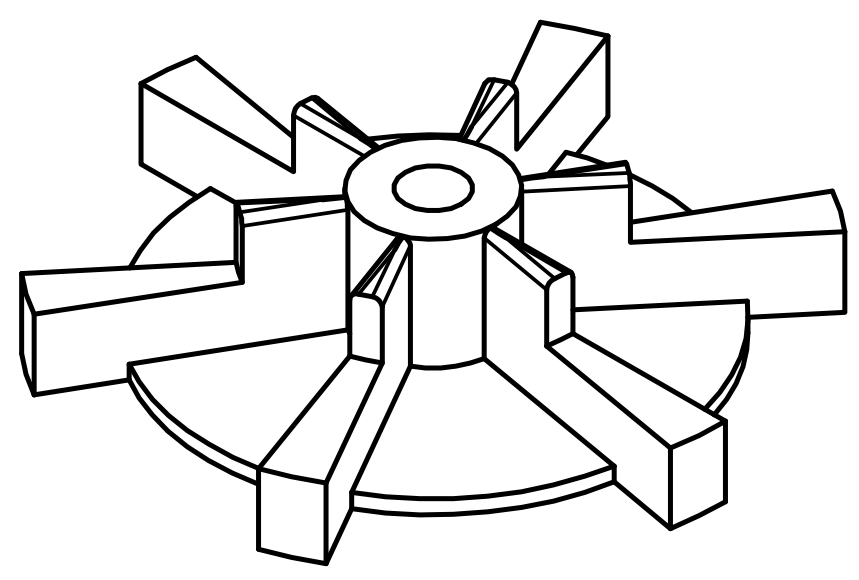

Scale 2:1
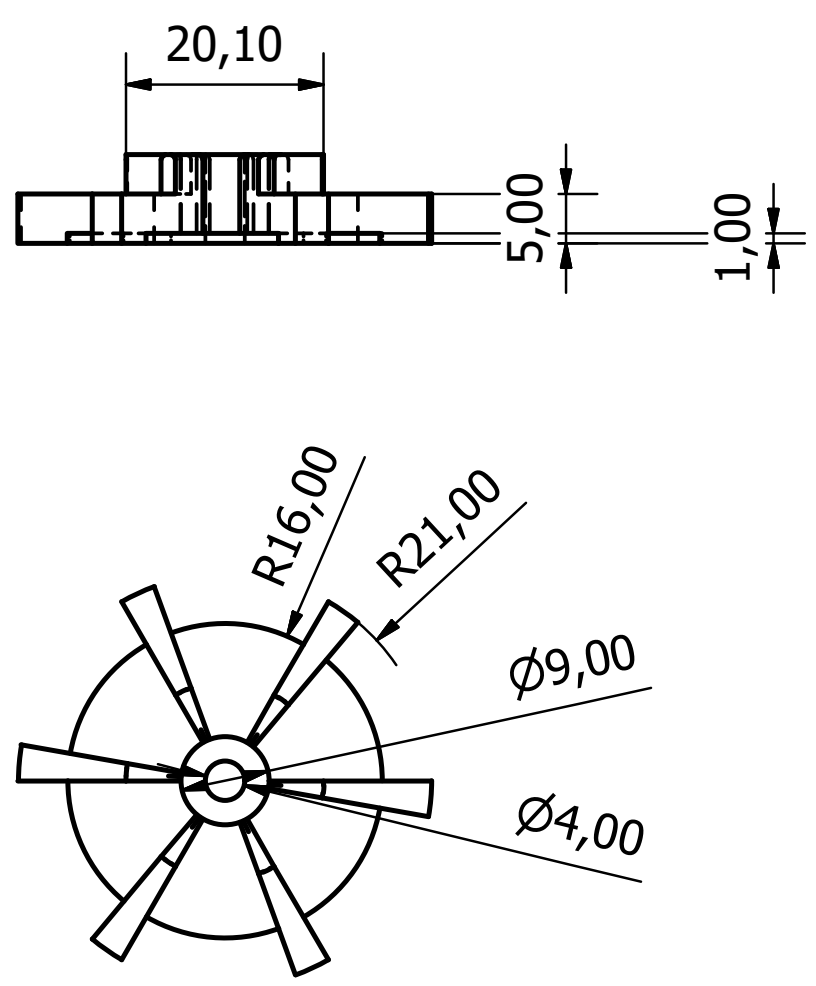

\begin{tabular}{|c|c|c|c|c|c|c|}
\hline \multirow[t]{2}{*}{\begin{tabular}{|l} 
Designed by \\
$L$
\end{tabular}} & Checked by & Approved by & Date & \multicolumn{2}{|c|}{ Date } & \\
\hline & & & Straight B & & Edition & $\begin{array}{r}\text { Sheet } \\
1 / 1\end{array}$ \\
\hline
\end{tabular}




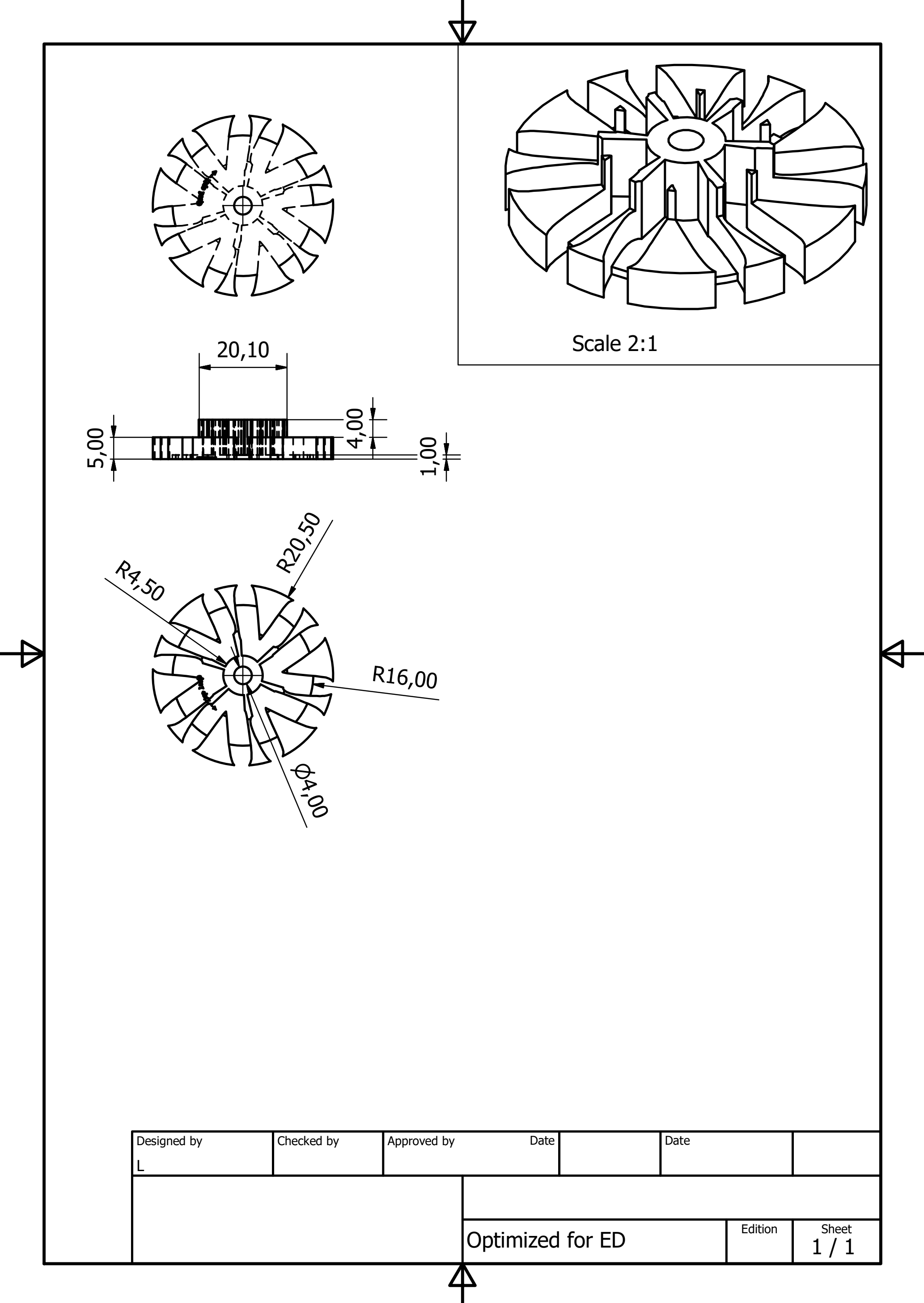




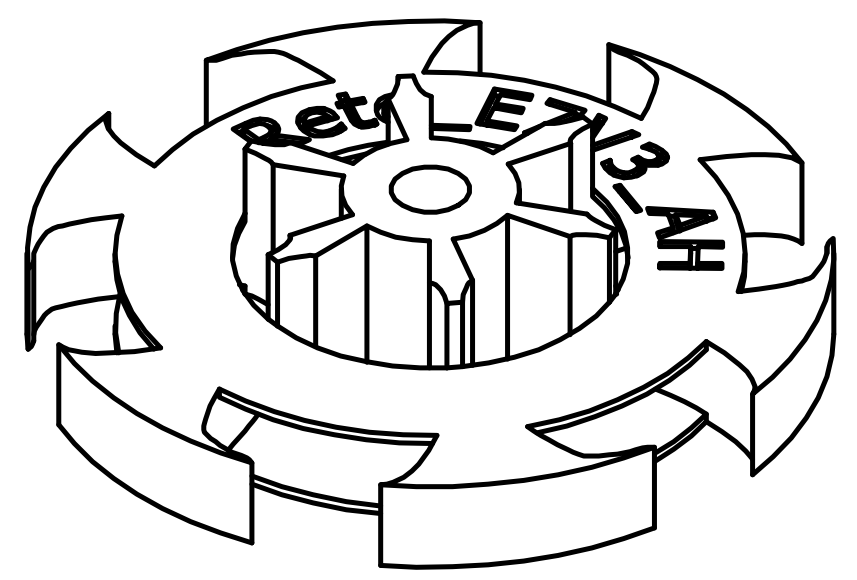

Scale 2:1
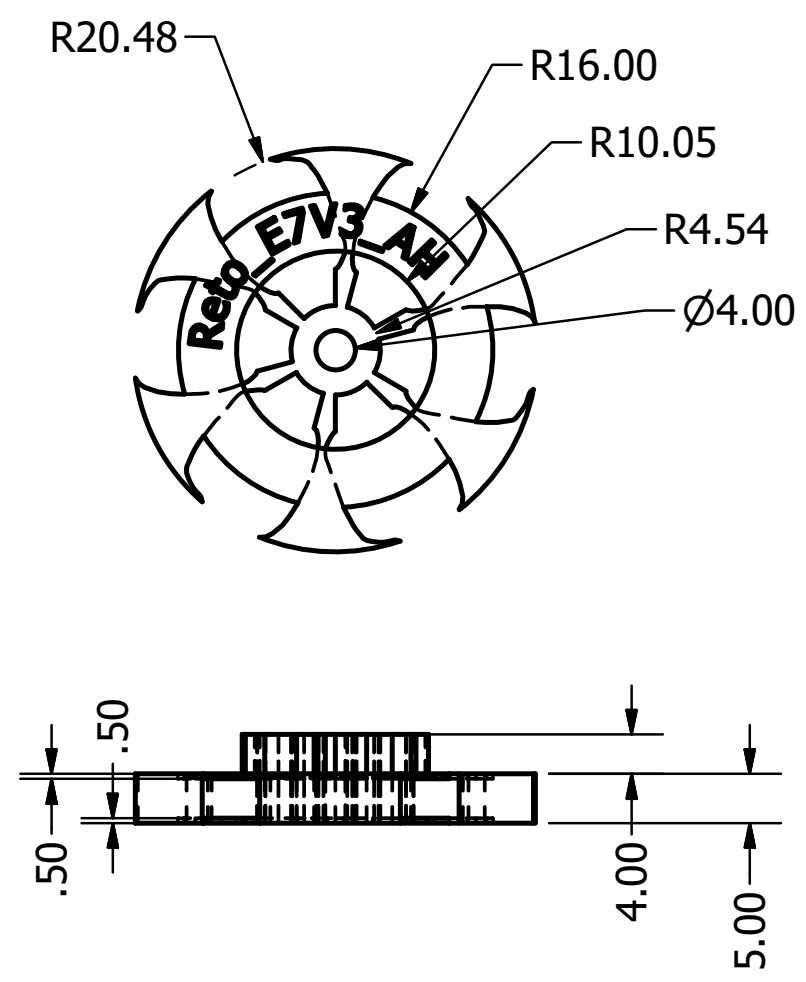

\begin{tabular}{|c|c|c|c|c|}
\hline \begin{tabular}{|l} 
DRAWN \\
L \\
CHECKED
\end{tabular} & $16 / 05 / 20$ & 16 & & \\
\hline & & TITLE & & \\
\hline $\mathrm{QA}$ & & & & \\
\hline MFG & & & & \\
\hline APPROVED & & & & \\
\hline & & SIZE & DWG NO & $\mathrm{REV}$ \\
\hline & & A4 & Rotor_reto_E7V3 $\mathrm{AH}$ iso & \\
\hline & & SCALE $1: 1$ & SHEET 1 OF 1 & \\
\hline
\end{tabular}

